\title{
Canadlian Journal of Surgery
}

Journal canadien

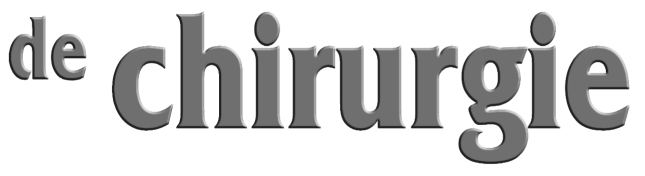

Vol. 57 (3 Suppl 3) June/juin 2014

canjsurg.ca

DOI: $10.1503 /$ cjs. 005714

\section{Trauma 2014}

Trauma Association of Canada (TAC) Annual Scientific Meeting

Centre Mont-Royal, Montréal, Que.

Thursday, Apr. 10 to Friday, Apr. 11, 2014 
Traumatic brain injury is not associated with hyperfibrinolysis. P. Veigas, B. Nascimento, J. Callum, L. Teodoro da Luz, J. Rezende Neto, S. Rizoli. From the Sunnybrook Health Sciences Centre, Toronto, Ont.

Background: Hyperfibrinolysis (HF) is uncommon after trauma but carries a high mortality. In practice, thromboelastometry (i.e., ROTEM) is the only test capable of diagnosing HF during initial resuscitation. We used ROTEM to study whether patients with traumatic brain injury (TBI) have HF on hospital admission. Methods: Review of all adult trauma patients with ROTEM on admission to Trauma Centre, grouped as: isolated TBI (Abbreviated Injury Score $[\mathrm{AIS}]$ head $\geq 2$ and non-AIS head $\leq 1$ ); no TBI (AIS head $\leq 1$ ) and concomitant TBI/non-TBI injuries. Hyperfibrinolysis was "lethal" if ROTEM clot lysis (CLI30) was abnormal or maximum lysis (ML) was $100 \%$, or nonlethal if ML was $99 \%$ or lower. Coagulopathy was abnormal ROTEM, international normalized ratio of 1.5 or more, or partial thromboplastin time of 40 seconds or more. Statistics used were the Student $t$ test and logistic regression. Results: A total of 974 patients (August 2011-December 2012) had admission ROTEM; 207 had isolated TBI; 447 had no TBI; 320 had both. Groups were similar except for significantly higher Injury Severity Score (26 admission ROTEM, 18 isolated TBI/13 non-TBI); more blood transfusion; more coagulopathy in concomitant TBI/non-TBI group. Lethal HF was diagnosed in 0 patients with isolated TBI; $0.44 \%$ nonTBI patients (mortality 100\%) and 5.9\% concomitant TBI/nonTBI injuries (mortality 63\%) $(p<0.001)$. Nonlethal HF was diagnosed in $36 \%, 40 \%$ and $30 \%$ (mortality $12 \%, 2.2 \%$ and $5.3 \%$, respectively, mostly of TBI). Injury Severity Score (OR 1.11, 95\% CI 1.08-1.14) was associated with HF but not TBI. Conclusion: Lethal HF though infrequent, carries prohibitive mortality (up to $63 \%$ ). No patient with isolated TBI had lethal HF on admission, while in others it was diagnosed in up to $5.9 \%$. Nonlethal HF was similarly diagnosed in all groups. ISS (but not TBI) was closely associated to HF suggesting that the extent of tissue destruction and not injury to specific tissues (i.e., brain) is the mechanism underlying hyperfibrinolysis in trauma.

Traumatic brain injury in the elderly: a level 1 trauma centre study. E. de Guise, H. Audrit, J. LeBlanc, J. Dagher, S. Tinawi, J. Marcoux, M. Maleki, M. Feyz. From the McGill University Health Centre, Montréal, Que.

Background: Mortality and morbidity due to brain injury in the elderly population is a growing clinical problem. The aim of this study was to investigate the characteristics and outcome of patients with traumatic brain injury (TBI) aged over 70 years old. Methods: Data were retrospectively collected on individuals $(n=$ 5778) who were admitted to the TBI program of the McGill University Health Centre from 2000 to 2012. Outcome measures used were the Extended Glasgow Outcome Scale (GOSE) as well as discharge destination. Results: The proportion of TBI patients who sustained a fall was higher in the 70+ age group $\left(\chi_{3}^{2}=672.951, p<0.001\right)$ and this older group showed more comorbidities (Kruskal-Wallis $\chi^{2}{ }_{1}=65.335, p<0.001$ ). In addition, the proportion of patients with severe TBI was significantly lower in the $70+$ age group. Also, the proportion of cases suffering multiple trauma was lower for this age group $\left(\chi^{2}{ }_{1}=88.144, p<\right.$ $0.001)$. Regarding outcome, as age of the patients increased, higher was the risk of having an unfavourable outcome as assessed with the GOSE (Spearman rank $r=-0.282, p<0.001$ ). Finally, the number of patients with TBI aged over 70 years was higher in the group discharged to long-term care $\left(\chi^{2}{ }_{4}=331.73, p<0.001\right)$. Conclusion: An increase in long-term care services dedicated to older individuals post-TBI suffering from multiple comorbidities will be needed in the future and fall prevention programs in seniors should be emphasized.

Chest drain insertion in the emergency department: compliance with guidelines in a university hospital emergency department. K. Tai, D. Yeung, J. Ollerton. From Nottingham University Hospitals Trust, Nottingham, United Kingdom

Background: Intercostal chest drains are often used in the emergency department, particularly in trauma. Advanced Trauma Life Support and British Thoracic Society guidelines are widely accepted nationwide in England but as with all invasive procedures, they can be associated with serious complications. The aim of this audit is to re-evaluate our current clinical practice; this was last evaluated in 2010. Methods: Adult patients were identified using our emergency department electronic records system (EDIS). Patients who had an intercostal drain (ICD) documented as a procedure between February 2012 and February 2013 were included in the audit. Results: Thirty-nine patients were identified, and $69 \%$ of chest drains were for trauma. The main proportion $(56 \%)$ were inserted by doctors specialist training year 3 or above, while there was no documentation of grade for $21 \%$. There was no documentation of consent in $44 \%$ of cases, $33 \%$ had documented verbal consent and $23 \%$ were unable to consent. Documentation including site, position, aseptic technique and drain size were variable. Of all identified cases $85 \%$ had documented technique of insertion. There were no documented postprocedure complications. Conclusion: All chest drains were inserted in the appropriate clinical setting. There was good clear documentation of indications and appropriate imaging when required. However, documentation of patient positioning, site, drain size, and aseptic technique were inconsistent. Documentation of consent was also missing on sizable occasions. Documentation of the procedures lacks standardization and details require prompting. As a result, we have developed and incorporated a checklist for documentation guidance in the chest drain kitbox.

Improving trauma care through the Rural Trauma Team Development Course (RTTDC) in Trinidad. A. Sorvari, ${ }^{*}$ J. Ali, ${ }^{\dagger}$ R. Adam, ${ }^{\ddagger}$ E. Ali. ${ }^{\S}$ From *St. Michael's Hospital, Toronto, Ont., †University of Toronto, Toronto, Ont., ¥SurgiMed, Trinidad and Tobago, and §Port of Spain General Hospital, Trinidad and Tobago.

Background: The Rural Trauma Team Development Course (RTTDC) is aimed at training personnel in under-resourced institutions to resuscitate, safely package and transfer trauma victims to definitive care in order to improve outcome. The program includes didactic lectures, case discussion scenarios, Communication and Performance Improvement and Patient Safety (PIPS) modules delivered at a level consistent with the educational background of the participants. In the developing country of Trinidad, most severely injured patients are transported directly to 1 of the 
9 main hospitals, but 105 lower level health centres and 7 district health facilities sometimes receive trauma patients. Conceivably, the RTTDC could impact on these patients' outcome by providing training as described. We assessed the feasibility and applicability of RTTDC training in Trinidad. Methods: An instructor orientation program (group F, $n=10$ ) was followed by an RTTDC provider program (group $S, n=16$ ) for caregivers from the district health centres. Mean pre- and post-multiple choice question (MCQ) scores on trauma resuscitation were compared by paired and unpaired $t$ tests, Educational value of the course, relevance of the PIPS, communication and scenario modules were evaluated using a 5-point Likert scale (1: strongly disagree, 2: disagree, 3: neutral, 4: agree, 5: strongly agree) with a $p$ value of 0.05 for statistical significance. General comments were also elicited. Results: Both groups improved their MCQ post-test scores (mean [SD] in 19 items) but group F scored higher in both tests (pretest: F-14.6 [1.4], S-10.9 [1.4]; post-test: F-16.7 [1.1], S13.6 [1.4]; $p<0.0002)$. The increase in scores were similar between groups (F-2.1 [1.1], S-2.6 [1.3]; $p=0.36$ ). Educational value: $100 \%$ of $\mathrm{F}$ and $\mathrm{S}$ strongly agreed/agreed that the objectives were met. The course was rated excellent/very good by $100 \%$ of $\mathrm{F}$ and $94 \%$ of S. Enthusiastic acceptance and potential for improving trauma outcome through the course were noted. Both $\mathrm{F}$ and $\mathrm{S}$ considered the PIPS, scenario and communication modules highly relevant. Conclusion: The RTTDC is strongly accepted in Trinidad for improving trauma care by training staff in peripheral centres that treat trauma patients. Inclusion of actual basic resuscitative skills (airway, hemorrhage, cervical spine control) in the scenarios was suggested.

Evaluation by stakeholders of the 9th edition Advanced Trauma Life Support (ATLS) instructor course. A. Sorvari, ${ }^{*}$ J. Ali, ${ }^{+}$Rasheed Adam, Ernest Ali. From *St. Michael's Hospital and tUniversity of Toronto, Toronto, Ont.

Background: Main changes in the 9th edition Advanced Trauma Life Support (2012) instructor course are "standing behind the student" concept, expanded content on feedback, introduction of mentor-mentee concept, omissions of: initial assessment (IA) demo and triage scenario discussion. The new course was evaluated by participants some of whom experienced and compared the old course. Methods: Nine courses (6 Canadian and 3 international) with 81 instructor candidates, 3 educators, 9 course directors, 9 coordinators and 27 instructors were analyzed using a 5 -point Likert scale with comments. Education content, meeting objectives, feedback and, mentor-mentee module, omission of discussion, and IA demo were rated (very poor, poor, good, very good or excellent). Skills improvement, application to courses, and superiority over the 8th edition (where applicable) were graded (strongly disagree, disagree, neutral, agree or strongly agree). Results: Grading was as follows by the different groups of participants: a) 81 instructor candidates (education content, objectives, feedback module, mentor-mentee [excellent, 81]; skills improvement and application [agree, 81]; IA demo-omission [very poor, 81]; comment — discussion module should be included); b) 3 educators (education content, objectives, feedback, mentormentee [excellent, 3]; improve skills, application to courses [strongly agree, 3]; comment, all recommend inclusion of discussion and IA demo); c) 9 course directors (education content [very good, 6; excellent, 3; improvement from 8th edition, 9]; feedback, mentor-mentee, objectives met [excellent, 9]; improvement over 8th edition [neutral, 2; agree, 7]; comment - retain IA and discussion module [9]); d) 9 coordinators (similar to c) except loss of triage [poor, 3; good, 6]); e) 27 instructors (educational content [good, 7; excellent, 20; improved over 8th edition: yes [20, no [7]; feedback, mentor-mentee [excellent, 27]; addition of discussion module [agree, 17; strongly agree, 10]; overall improvement [neutral, 3; agree, 20; strongly agree, 4]; recommend — retain IA demo [27]). Conclusion: The 9th edition was considered significantly improved and highly rated by participants but formal inclusion of a discussion component and the initial assessment demo modules was highly recommended.

\section{Pediatric trauma systems. I. Bratu, C. Wang, C. Falconer. From the Stollery Children's Hospital, University of Alberta, Edmonton, Alta.}

Background: Our objectives are to define the characteristics of pediatric trauma systems across Canada, understand access to pediatric trauma systems, assess variability of pediatric trauma systems, highlighting strengths and challenges. Methods: The REDCap survey was electronically sent to all Canadian pediatric trauma coordinators and medical directors as an anonymous cross sectional self-report. Results: Twenty-one (out of a possible 35) participants responded to our survey. Almost all are level 1 pediatric trauma centres, but most are not TAC accredited, yet maintain pediatric trauma databases and quality assurance programs. All have designated pediatric trauma team membership, and activation guidelines, as well as prehospital communication and transport protocols/algorithms. Most feel that the trauma team is undercalled. Most do not have an in-house 24/7 operating room (OR) team, but the OR can be reached and called back after hours. Most have a pediatric surgical training program with pediatric surgery fellows with a high reliance on pediatric surgery fellows for pediatric trauma team coverage during working hours and on call. There is no reliable funding for trauma team leaders on call program by local health authorities. There are some gaps in the pediatric trauma on-call coverage for the residents. Three quarters have good access to rehabilitation services. Around a third stated that the pediatric trauma program is not defined and recognized in the administrative structure as a distinct program. Conclusion: Knowledge of successes and challenges of the current state of our pediatric trauma systems will create discussion, and focus on improvement and advocacy.

The serious impact of bicycle handlebar injuries. H. Cerniawsky, I. Bratu, T. Rankin, B. Sevcik. From the Stollery Children's Hospital, The University of Alberta, Edmonton, Alta.

Background: Handlebar injuries are common and severe yet can be overlooked due to their insidious presentation. We seek to investigate the epidemiology of bicycle trauma, particularly handlebar injuries and their diagnosis. Methods: Complementary databases were used to investigate 462 participants under 17 years presenting to a children's emergency room (ER) following a bike injury between February 2012 and July 2013. Results: Abdominal handlebar injuries represented $9 \%$ of bicycle injuries with 1 in 5 resulting in damage to internal organs compared with 1 in 100 of nonhandlebar injuries. A total of $10.9 \%$ of handlebar and 
only $1.2 \%$ of nonhandlebar injuries had Injury Severity Score over 12 . Handlebar trauma accounted for $45.4 \%$ of solid, $87.5 \%$ of hollow, $66.6 \%$ of vascular or lymphatic and $100 \%$ of pancreatic organ injuries. Clinical features of emesis, fever, peritonitis and elevated lipase were suggestive of internal organ damage in patients with a history of handlebar trauma. A total of $55.5 \%$ of children presenting with a handlebar to abdomen were initially misdiagnosed at the treating ER and sent home, only to return to definitive care, with an average delay of 18.3 hours, and a mean hospital stay of 6.75 days. Conclusion: The mechanism of handlebar injury to the abdomen in a child presenting to the ER is by itself an indicator of potential severe injury and requires serial abdominal exams, laboratory investigations, and prompt CT abdomen to rule out hollow viscus or solid organ injuries. Caregivers should seek health care if emesis, peritonitis, or fever appears following a handlebar injury. Modifications to handlebars may reduce the severity and number of injuries.

Safety pneumothoraces in ventilated pediatric trauma patients: a review. C. Fulton, ${ }^{*}$ I. Bratu. ${ }^{\dagger}$ From the * Stollery Children's Hospital and the TUniversity of Alberta, Edmonton, Alta.

Background: Blunt occult pneumothoraces that are observed may require in $4 \%$ of pediatric cases and in $31 \%$ of adult cases placement of an eventual chest tube for progression. We seek to understand our own institution's management of severely injured children with pneumothoraces, especially occult ones. Methods: A retrospective chart review was conducted of the Alberta Trauma Registry of all ventilated pediatric patients at a Children's Hospital from Jan. 1, 2001, to Dec. 31, 2011, with Injury Severity Score over 12 and a diagnosis of either hemo- or pneumothorax. Results: There were 1334 severely injured children, with 392 admitted to the pediatric intensive care unit (ICU) and ventilated. Of those intubated, 90 children (mean age $12.7 \mathrm{yr}$ ) had an initial pneumothorax. A total of 125 pneumothoraces were identified and 65 children received a total of 123 chest tubes. Thirty children were initially treated without a chest tube, but 5 progressed (17\%) requiring a chest tube. Twenty-two children had complications with their chest tubes, with most being minor (e.g., pulling back the chest tube or a small persistent pneumothorax after chest tube removal). However, 12 children needed at least 1 replacement chest tube. Conclusion: Pneumothoraces in severely injured children who are ventilated in the ICU can occur in almost a quarter of cases. Occult pneumothoraces can be safely observed with serial exams or chest $\mathrm{X}$-rays and if they progress, timely chest tube insertion may be needed. Chest tube placement is usually accomplished with few complications, but continued education about proper chest tube placement is encouraged.

Extended fast (EFAST): Is it the time to be an adjunct in ATLS? Y. Abdulrahman, ${ }^{*}$ S. Musthafa, ${ }^{\dagger}$ S.Y. Hakim, ${ }^{*}$ S. Nabir, ${ }^{+}$A. Qanbar, ${ }^{*}$ I. Mahmood, ${ }^{*}$ T. Siddiqui, ${ }^{*}$ W.A. Hussein, ${ }^{+}$H.H. Ali, ${ }^{*}$ I. Afifi, ${ }^{*}$ A. El-Menyar, ${ }^{*} \neq$ H. Al-Thani. ${ }^{*}$ From the *Hamad General Hospital, the tUniversity of Qatar, and the \#Will Cornell Medical College, Doha, Qatar

Background: Use of ultrasound for abdominal trauma (FAST) began in Europe in 1970 and was accepted as an adjunct to pri- mary survey in the Advanced Trauma Life Support (ATLS) as late as 1997. This is to test whether we will have enough evidence to adopt extended FAST (EFAST) as a new adjunct in the ATLS algorithm and to prevent unnecessary or even a delay in chest tube insertion. Methods: This is a single-blinded prospective study (July 2011-January 2013). Data was collected at Hamad level 1 Trauma Centre. The inclusion criteria were any adult patient with a blunt chest trauma who needed to be further investigated by a chest CT scan according to ATLS guidelines. The exclusion criteria were any penetrating trauma or a thoracostomy tube insertion before CT chest scanning. Results: We included 305 patients aged between 18 and 75 years, 298 (98\%) males. The chest clinical examination sensitivity was $48.6 \%$, specificity of 95.7\%. The positive predictive value (PPV) was $91 \%$ and a negative predictive value (NPV) was $67.5 \%$. CXR sensitivity was $17.3 \%$ with a specificity of $99.8 \%$, with a PPV of $92.8 \%$ and a NPV of $89.1 \%$. The EFAST sensitivity was $42.7 \%$, specificity of $92.8 \%$, a PPV of $43.8 \%$ and a NPV was as high as $92.4 \%$. Conclusion: The high specificity (92.8\%) and NPV (92.4\%), and the score of $72 \%$ of the missed cases was $\geq 20$. This study gives more evidence and encouragement for the use of EFAST as an important adjunct in ATLS.

Determinants of access to an integrated trauma system: a population-based cohort study. B.K. Brice Lionel, L. Moore, ${ }^{*}$ B. Cisse, ${ }^{*}$ M. Gagné, ${ }^{\dagger}$ G. Bourgeois, ${ }^{*}$ J. Lapointe. ${ }^{*}$ From the *Université Laval, Laval, Que., tInstitut national de santé publique, and the flnstitut national d'excellence en santé et en services sociaux, Quebec, Que.

Background: Access to specialized trauma care for trauma patients is an important measure of trauma system efficiency. However, little data are available on access to integrated trauma systems. We aimed to describe access to trauma care in an integrated trauma system and identify its determinants. Methods: We conducted a population-based cohort study using an administrative hospital discharge database. We included all injured adults admitted to acute care hospitals in the province of Québec between 2006 and 2011. Proportions of injured patients transported directly or transferred to a trauma centre were assessed. Determinants of access were identified through a modified Poisson regression model and relative importance analysis was used to determine the relative importance of each independent variable. Results: Of the 136653 injury admissions selected, $75 \%$ were treated within the trauma system. The proportion of access was $90 \%$ for major injuries (ICD-9 Injury Severity Score < 0.85 ), $78 \%$ for patients aged under 65 years and $70 \%$ for patients aged 65 years or older. Access was higher among patients living in more remote areas (trend $p<0.001$ ). Among patients with major trauma, the region of residence followed by mechanism of injury, number of trauma diagnoses, injury severity and age were the most important determinants of access to trauma care. Conclusion: In an integrated, mature trauma system, we observed high access to trauma care. However, problems in access to care emerged for elderly and in urban areas. Access to care should be monitored as part of quality of care improvement activities and prehospital guidelines for trauma patients should be applied uniformly throughout the province. 
Impact of socioeconomic status on unplanned readmission following a traumatic injury. B. Cisse, ${ }^{*}$ L. Moore, ${ }^{*}$ B.K. Brice Lionel, ${ }^{*}$ A. Boutin, ${ }^{*}$ A. Lavoie, ${ }^{\dagger}$ G. Bourgeois. ${ }^{*}$ From the *Université Laval and the tHôpital de l'EnfantJésus, Québec, and the flnstitut national d'excellence en santé et en services sociaux, Montréal, Que.

Background: In US Medicare populations, the 30-day readmission rate was $19.6 \%$ in 2009 with an estimated cost of $\$ 17$ billion. Despite the recognized impact of socioeconomic status (SES) on readmission in other patient populations such as cardiovascular disease, its impact on readmission in trauma patients is unclear. This study aimed to examine the effect of SES on unplanned readmission following a traumatic injury in a setting with universal health insurance. Methods: We conducted a multicentre cohort study involving the 56 adult trauma centres in Canadian provincial trauma system between 2005 and 2010. The SES was determined by ecological measures of material and social deprivation. Odds ratio (OR) of readmission and $95 \%$ confidence intervals adjusted for covariates were generated using multivariate logistic regression with a correction for hospital clusters. Results: The cohort consisted of 52122 patients with a readmission rate of $6.5 \%$. Social deprivation was associated with increased odds of unplanned readmission (OR 1.07, 1.06, 1.16, 1.20 for quintiles 2-5 compared with quintile 1 ). The risk of unplanned readmission didn't increase significantly with material deprivation (OR 1.03, 0.99, 1.06, 1.09 for quintiles 2-5 compared with quintile 1). Conclusion: Patients admitted for traumatic injury who suffer from social deprivation have an increased risk of unplanned rehospitalization within 30 days of discharge. This research suggests that such patients may benefit from discharge and follow-up interventions to ensure that the risk for readmission is minimized.

Influence of socioeconomic status on trauma centre performance evaluations based on unplanned readmission in a Canadian trauma system. B. Cisse, ${ }^{*}$ L. Moore, ${ }^{*}$ B.K. Brice Lionel, ${ }^{*}$ A. Boutin, ${ }^{*}$ A. Lavoie, ${ }^{\dagger}$ G. Bourgeois. ${ }^{*}$ From the *Université Laval, tHôpital de I'Enfant-Jésus, Québec, and the flnstitut national d'excellence en santé et en services sociaux, Montréal, Que.

Background: Unplanned readmission has recently been validated as a quality indicator (QI) to evaluate trauma care. Socioeconomic status (SES) is known to be an important determinant of unplanned readmission. However, it is rarely accounted for in QI. This study aimed to determine if accounting for inter-hospital variation in SES has an impact on the results of trauma centre performance evaluations based on unplanned readmission in a universal access health system. Methods: This study was based on adults discharged from the 56 trauma centres in Quebec between 2005 and 2010. We calculated rates of readmission for each hospital adjusted for age, sex, etc. (original QI) and rates adjusted for all these factors plus SES (SES-adjusted QI). The agreement between the QI and the SES-adjusted QI was assessed using correlation coefficients. Results: The cohort comprised 52122 patients with a readmission rate of $6.5 \%$. The proportion of patients in the highest quintile of social and material deprivation varied from $0 \%-44 \%$ and $0 \%-91 \%$ across hospitals, respectively. We observed a strong correlation between original and
SES-adjusted rates of unplanned readmission $(r=0.99)$. Conclusion: Despite being an important determinant of readmission and subject to important inter-hospital variation, the addition of SES didn't influence the results of trauma centre performance evaluations based on unplanned readmission. Results suggest that QI based on unplanned readmission do not require adjustment for SES in a universal access health care system.

A quality indicator to benchmark 30-day unplanned hospital readmission for trauma admissions. L. Moore, ${ }^{* \dagger}$ H.T. Stelfox, ${ }^{\ddagger}$ A. Turgeon, ${ }^{*}$ A. Nathens, ${ }^{\S}$ A. Lavoie, ${ }^{\S \uparrow}$ G. Bourgeois, ${ }^{\S \Phi} \mathrm{J}$. Lapointe. ${ }^{\delta \rrbracket}$ From the *Université Laval and the tAxes de Santé des populations et pratiques optimales en santé (Population Health - PracticeChanging Research Unit) et Unité de traumatologieurgence-soins intensifs, Centre de recherche du $\mathrm{CHU}$ de Québec et Hôpital de I'Enfant-Jésus, Laval, Que., the ‡University of Calgary, Calgary, Alta., §St. Michael's Hospital, Toronto, Ont., and ПInstitut national d'excellence en santé et en services sociaux, Montréal, Que.

Background: Unplanned readmissions represent 20\% of all admissions and increase patient morbidity and resource use. Our objective was to derive and internally validate a quality indicator (QI) for 30-day unplanned hospital readmission following hospitalization for injury. Methods: This study is based on a multi-centre retrospective cohort from a Canadian provincial trauma system (2005-2010; 57 trauma centres; $n=57$ 524). Data were abstracted from the provincial trauma registry and linked to the hospital discharge database. The primary outcome was unplanned readmission to an acute care hospital within 30 days of discharge. Candidate risk factors were identified by expert consensus and selected for derivation of the risk adjustment model using bootstrap resampling. The validity of the QI was evaluated in terms of inter-hospital discrimination, construct validity, and forecasting. Results: The risk adjustment model includes patient age, sex, Injury Severity Score, region of the most severe injury, and 11 comorbid conditions. The QI discriminates well across trauma centres (coefficient of variation $=$ 0.02 ), is correlated with QI that measure hospital performance in terms of structure $(\mathrm{r}=-0.24)$ and clinical processes $(\mathrm{r}=$ $-0.38)$, risk-adjusted mortality $(\mathrm{r}=0.32)$ and complication rates $(r=0.38)$. Conclusion: We have developed a QI based on riskadjusted 30-day rates of unplanned readmission that can be used to evaluate trauma care with routinely collected data. The QI is based on a comprehensive risk adjustment model with good internal and temporal validity, and demonstrates good properties in terms of discrimination, construct validity, and forecasting. This research represents an essential step toward reducing unplanned readmission rates to improve resource use and outcomes following injury.

A quality indicator to benchmark acute care length of stay for trauma admissions. L. Moore, ${ }^{*}$ H.T. Stelfox, ${ }^{\dagger}$ A. Turgeon, ${ }^{\neq}$A. Nathens, ${ }^{\S}$ G. Bourgeois," J. Lapointe," M. Gagne," A. Lavoie." From the *Université Laval, Laval, Que., the TUniversity of Calgary, Calgary, Alta., the ¥Unité de traumatologie-urgence-soins intensifs, Centre de Recherche FRSO du CHA (Hôpital de I'Enfant-Jesus), Québec, Que., the §Sunnybrook Health Sciences Centre, 
Toronto, Ont., and the IInstitut national d'excellence en santé et services sociaux, Quebec, Que.

Background: Injury is second only to cardiovascular diseases in terms of acute care resource use but no Quality Indicator (QI) is currently available to evaluate trauma care in terms of length of stay (LOS). Our objective was to derive and internally validate a QI for acute care length of stay (LOS) following admission for injury. Methods: The study is based on a multi-centre retrospective cohort from a Canadian provincial integrated trauma system (2005-2010; 57 trauma centres, $n=57524)$. Data were abstracted from the provincial trauma registry and linked to the hospital discharge database. Candidate risk factors were identified by expert consensus and selected for model derivation using bootstrap resampling. The validity of the QI was evaluated in terms of inter-hospital discrimination, construct validity, and forecasting. Results: The risk adjustment model includes age, injury mechanism, maximum Abbreviated Injury Scale score for each body region injured, the Glasgow Coma Score, components of the Elixhauser comorbidity index, the number of admissions in the 12 months before injury, and transfer status. The model explains $37 \%$ of the variation in LOS. The QI discriminates well across trauma centres (coefficient of variation: $0.02,95 \%$ CI $0.011-$ $0.028)$ and is correlated with QI on processes of care $(\mathrm{r}=-0.32)$, complications $(\mathrm{r}=0.66)$, unplanned readmissions $(\mathrm{r}=0.38)$, and mortality $(r=0.35)$. Conclusion: We propose a QI based on risk-adjusted LOS to evaluate trauma care that can be implemented with routinely collected data. This QI can be used to target interventions to reduce LOS, which will lead to more efficient resource use and may improve patient outcomes following injury.

Donabedian's structure-process-outcome quality of care model: validation in an integrated trauma system. L. Moore, ${ }^{*}$ A. Lavoie, ${ }^{+}$G. Bourgeois, ${ }^{+} \mathrm{J}$. Lapointe, ${ }^{+}$ $X$. Nevue. ${ }^{*}$ From the *Université Laval, Laval, the tInstitut national d'excellence en santé et services sociaux and the ¥Unité de traumatologie-urgence-soins intensifs, Centre de Recherche FRSO du CHA (Hôpital de I'EnfantJesus), Québec, Que.

Background: According to Donabedian's healthcare quality model, improvements in the structure of care should lead to improvements in clinical processes, which should improve patient outcome. This model has been widely adopted by the trauma community but has not yet been validated in a trauma system. We aimed to assess the performance of an integrated trauma system in terms of structure, process and outcome and evaluate the correlation between quality domains. Methods: Quality of care was evaluated for patients with major trauma treated in a Canadian provincial trauma system (2005-2010; 57 centres; 11183 patients). Structural performance was measured according to American College of Surgeons accreditation criteria. Process performance was assessed by average conformity to 15 process QIs. Outcome performance was measured using risk-adjusted rates of mortality, complications, and readmission. Correlation between QI was assessed with Pearson's correlation coefficients. Results: Statistically significant correlations were observed between structure and process QIs $(\mathrm{r}=0.29)$, and process and outcome QIs $(\mathrm{r}=$ -0.22 for mortality, $r=-0.48$ for complications, and $r=-0.30$ for readmission). Weaker correlations were observed between struc- ture and outcome QIs $(r=-0.19$ for mortality, $r=-0.29$ for complications). Strong significant positive correlations were observed between outcome QIs $(r=0.69$ for mortality-readmission; $r=$ 0.67 for complications-readmission; $r=0.87$ for mortalitycomplications). Conclusion: Significant correlations between quality domains observed in this study suggest that Donabedian's model is a valid model for evaluating trauma care. It suggests that trauma centres that perform well in terms of structure also tend to perform well in terms of clinical processes, which in turn has a favourable impact on patient outcomes.

Which hospital complications should be used to evaluate the quality of adult trauma care? An expert consensus study. L. Moore, ${ }^{*}$ H.T. Stelfox, ${ }^{+}$A. Turgeon, ${ }^{\neq}$F. Lauzier, ${ }^{*}$ N. Le Sage, ${ }^{*} M$. Shemilt. ${ }^{*}$ From the * Université Laval, Laval, Que., the HUniversity of Calgary, Calgary, Alta., and the $¥$ Unité de traumatologie-urgence-soins intensifs, Centre de Recherche FRSO du CHA (Hôpital de I'EnfantJesus), Québec, Que.

Background: Complications affect up to $37 \%$ of patients hospitalized for injury and increase mortality, morbidity, and costs. We aimed to develop a consensus-based list of complications that can be used to assess the acute phase of adult trauma care. Methods: We conducted a 3-round Web-based Delphi survey among experts with a broad range of clinical expertise and geographical diversity. In the first round, participants were asked to list all complications that could be used to evaluate trauma care. In the subsequent 2 rounds, they were asked to rate the importance of each complication. The main outcome measure was median importance rating on a 5-point Likert scale (very low-very high); complications with a median $\geq 4$ and no disagreement were retained. A secondary measure was the perceived quality of information available in patient files for each complication. Results: Of 19 experts invited to participate, 16 (84\%) completed all rounds. Of 73 complications suggested in round 1, 25 were retained including acute respiratory distress syndrome, hospitalacquired pneumonia, sepsis, acute renal failure, deep vein thrombosis, pulmonary embolism, wound infection, decubitus ulcers, and delirium. Of these, 19 (76\%) were perceived to have high or very high quality information in patient files by more than $50 \%$ of panel members. Conclusion: This study proposes a consensus-based list of complications that can be used to evaluate acute adult trauma care. These complications can be used to develop an informative and actionable quality indicator with the goal of decreasing rates of hospital complications and thus improving patient outcomes and resource use.

Trends in hospitalization associated with TBI in an urban level 1 trauma centre. H. Audrit, M. Feyz, J. LeBlanc, J. Dagher, S. Tinawi, J. Lamoureux, J. Marcoux, M. Maleki, E. de Guise. From the McGill University Health Centre, Montréal, Que.

Background: Traumatic brain injury (TBI) is the single largest cause of death and disability following injury worldwide. The aim of this study was to determine the trends of characteristics associated with TBI patients hospitalized in an urban level 1 trauma centre. Methods: Data were retrospectively collected on those individuals $(n=5778)$ who had been admitted in the Traumatic 
Brain Injury Program of the McGill University Health Center (MUHC-MGH) from 2000 and 2012. Results: A regression showed a significant upwards trend in the yearly number of cases as well as an upward trending by year in the proportion of TBI cases aged 70 years old or more. The Injury Severity Scores were positively associated with the year indicating a slight increase in injury severity over the years and there was an increase in patient psychological, social and medical premorbid complexity. In addition, the extended Glasgow Outcome Scale score tended to decrease slightly over the years (become more severe) and there was a slight decrease in the proportion of discharges home and in the proportion of death. Conclusion: These results will help to understand the impact of the TBI in an urban Canadian level 1 trauma centre. These findings can also provide information to help health stakeholders plan for future resources and it should be used to develop public prevention strategies and educate the community about the risk of TBI.

Validation and interrater reliability of trauma registry data in a level 1 trauma centre. M. Mercado, A. Rados, J. Xiao, S. Lally, D. Young. From the Alberta Health Services, Calgary, Alta.

Background: The value of data contained in a trauma registry depends on its quality. Thus, data collected needs constant reassessment to ensure its quality. Herein, we report the process of data validation and interrater reliability among 4 data analysts (DAs) in a regional trauma centre. Methods: From all severely injured patients (Injury Severity Score $\geq 12$ ), data elements of 12 sections were abstracted from each patient's records and manually entered into the trauma registry using Collector ${ }^{\mathrm{TM}}$. Data validation was done for the previous fiscal year (2012-2013). Interrater reliability was performed monthly on 1 section of data elements at a time. Records from 10 patients were randomly selected, and the DAs entered the data elements independently. Results were discussed and analyzed. Results: Validation detected 493 from 420900 elements $(0.12 \%)$ of 915 patients in year 2011-2012, and 199 from 529000 elements (0.04\%) of 1150 patients in year 2012-2013 for correction. These inaccuracies included uncompleted fields, interpretation and random errors particularly from Section II - Injury Data. Interrater reliability for Section II of 30 patients was performed and 19 out of 21 elements were reached $80 \%-100 \%$ consensus, which was improved from the first 10 patients, although "Incident time" is still a challenge. Conclusion: Validation plays a critical role in minimizing errors in a Trauma Registry database. An efficient on-going program for data validation should be developed in a trauma registry. Interrater reliability evaluation among multiple DAs is integral to optimal registrar proficiency.

Assessing the "time to CT scan" and outcomes in moderate to severe traumatic brain injury (TBI). S. Sobrian-Couroux, ${ }^{*}$ A. McFarlan, ${ }^{\dagger}$ S. Rizoli. ${ }^{\dagger}$ From the *Sunnybrook Health Sciences Centre, and tSt. Michael's Hospital, Toronto, Ont.

Background: Time to computed tomography (CT) scan is a major quality indicator for evaluating traumatic brain injury (TBI) care. The consensus among traumatologists is to perform head CT scans as soon as possible, preferably within 1 hour of injury, on all patients with significant head injury. These recommendations are consistent with TAC and other guidelines. Methods: Retrospective review of all isolated moderate to severe TBI admissions to a single trauma centre between July 2010 and June 2013. Time to CT scan was evaluated and the factors affecting this time were compared between patients undergoing trauma team activation (TTA) versus nontrauma activation. We analyzed outcome measurements and disposition status. Results: Ninety patients were analyzed. Males are 5 times more likely to incur TBI than females. A total of $65.5 \%$ of the admissions were TTA and $59 \%$ arrived directly from the scene. Overall the average time to CT from admission is 4 hours and 36 minutes (Figure). A total of $28.9 \%$ of the patients expired in hospital. Trauma activations had $65.4 \%$ mortality. Conclusion: Nontrauma activation admissions experienced longer wait times for CT and were mainly referrals. A total of $65.8 \%$ arrived with CT completed within 4.5 hours of admission from the referring facility.

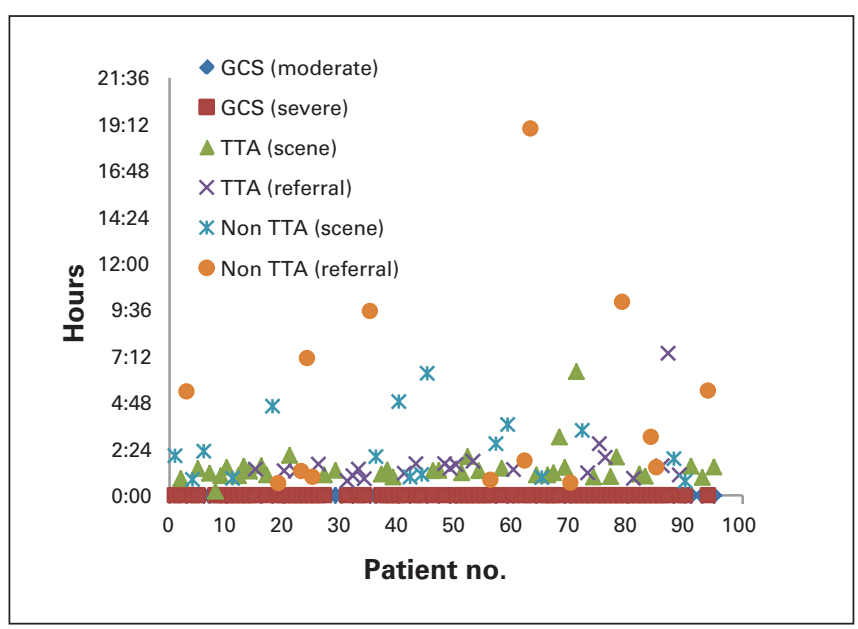

There's no place like home: implementation of a home safety device program. J. Harrington, T. Charyk Stewart, L. Wolfs, B. Batey. From the London Health Sciences Centre, London, Ont.

Background: Injuries are the number 1 health concern for children. Each year nearly 3000 children come to London Health Sciences Centre for treatment of an injury in the home; the most common reason children visit our emergency department. Children are injured in falls by slipping, tripping and falls down stairs. Although many parents feel home is the safest place for their children, they are at significant risk for injury. Methods: To provide a prevention program to new families to reduce the incidence of home injuries in London and area. This is the first hospital-based home injury prevention program in Ontario. Using a multidisciplinary approach provide safety devices along with home safety education to first time families and children $(<2)$ injured in the home. Follow up with families occurred 8 months post-delivery. Results: Implementation of program in areas of a birthing centre or trauma centre in April 2013. In the first 6 months of the program, 900 home safety device kits were given away. Based on previous data we should reach approximately 1500 new families per year and 200 infants in the emergency department. Conclusion: Evaluation is ongoing, and initial positive response demonstrates that the implementation of an innovative home 
safety device program is a beneficial and positive approach to helping prepare first time families for the arrival of their baby. Our goal is to ultimately reduce the incidence of home related injuries to infants.

Early weight-bearing and mobilization versus nonweight-bearing and immobilization after surgical fixation of unstable ankle fractures: a randomized controlled trial. N. Dehghan, ${ }^{*}$ R. Jenkinson, ${ }^{\dagger}$ M. McKee, ${ }^{\ddagger}$ E. Schemitsch, ${ }^{\ddagger}$ A. Nauth, ${ }^{\neq}$J. Hall, ${ }^{\neq}$D. Stephen, ${ }^{\dagger}$ H. Kreder. ${ }^{\dagger}$ From the *University of Toronto, tSunnybrook Health Sciences Centre, and ¥St. Michael's Hospital, Toronto, Ont.

Background: The optimal post-operative protocol with respect to weight-bearing and ankle range of motion (ROM) following surgical fixation of acute ankle fractures remains controversial. Convention dictates non-weight-bearing and immobilization for 6 weeks post-operatively. However early weight-bearing may expedite return to function, with the potential risk of loss of fixation or wound complications. Methods: We conducted a multicentre randomized controlled trial. Patients who underwent acute surgical fixation of an unstable ankle fracture were randomized to one of 2 rehabilitation protocols: 1) early weight-bearing (weightbearing and ankle mobilization at 2 weeks) or 2) delayed weightbearing (non-weight-bearing and casting for 6 weeks). The primary outcome was rate of return to work at 6 weeks. Secondary outcomes included ankle ROM, SF-36 heath outcome scores, Olerud/Molander ankle function score, and rates of complications. Results: In total 110 patients were recruited: 56 randomized to early weight-bearing and 54 randomized to the delayed weight-bearing group. There were no differences between the 2 groups with regards to patient characteristics. There was no statistical difference between the 2 groups with regards to rate of return to work at any time point. However, at 6 weeks post-op, patients in the early weight-bearing group had significantly improved ankle ROM ( $42^{\circ}$ v. $\left.28^{\circ}, p=0.001\right)$; Olerud/Molander ankle function scores ( 44 v. $31, p=0.002$ ), and SF-36 scores on both the physical $(50$ v. $42, p=0.008)$ and mental $(62$ v. $54, p=$ 0.005 ) components. There were no cases of fixation failure, and no differences with regards to wound complications or infection between the groups. Conclusion: Given the convenience for the patient, the early improved functional outcome, and the lack of an increased complication rate with early weight-bearing, we recommend early post-operative mobilization and weight-bearing in patients with surgically treated ankle fractures.

Temporal trends and differences in trauma centre hospital mortality across Ontario from 2005 to 2011. D. Gomez, ${ }^{*}$ A. Alali, ${ }^{*}$ B. Haas, ${ }^{+}$H. Tien, ${ }^{\ddagger}$ A. Nathens. ${ }^{\ddagger}$ From the *University of Toronto, †St. Michael's Hospital, and the ¥Sunnybrook Health Sciences Centre, Toronto, Ont.

Background: The primary objective of this analysis was to evaluate differences in trauma centre (TC) mortality over time. Secondarily, we explored TC-specific mortality to determine the extent of variation across centres. Methods: Data on 26421 adults admitted to a TC from 2005 to 2011 were derived from the Ontario Trauma Registry. Statistical models were used to estimate risk-adjusted mortality over time and TC-specific mortality. To quantify variability between TCs, median odds ratios (MORs) were calculated. Adjusted odds of death were calculated for each TC to identify centres with higher (black), average (grey), and lower than expected mortality (white) (Figure). Results: Mortality decreased from $13.2 \%$ in 2005 to $11.2 \%$ in 2011 . After riskadjustment, the odds of death decreased by $3 \%$ (95\% CI 0\%-5\%) per year. TC-specific mortality ranged from $11.4 \%$ to $13.1 \%$ with a MOR of 1.25, suggesting that the odds of dying were 1.25fold greater if the same patient was admitted to one randomly selected TC as opposed to another. Differences were greatest for patients with head injuries and elderly patients as evidenced by higher MORs and number of outliers. Conclusion: Differences in mortality across TCs were identified, which was greatest among elderly patients and those with traumatic brain injuries. System-wide performance improvement should focus on the elderly and patients with isolated head injuries.

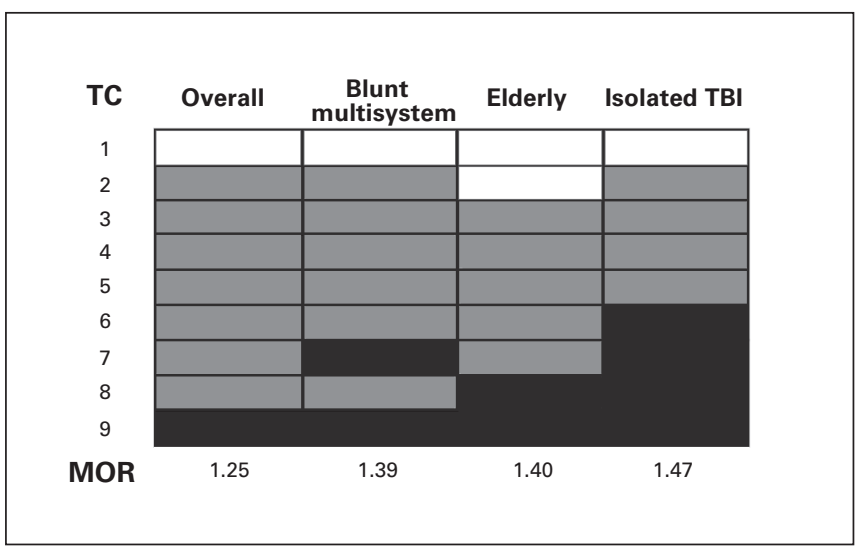

Barriers to implementing the world health organization's trauma care checklist: a Canadian pilot experience. B. Nolan, ${ }^{*}$ R. Zakirova, ${ }^{\dagger}$ J. Bridge, ${ }^{\ddagger}$ A. Nathens. ${ }^{\dagger}$ From the *University of Toronto, †St. Michael's Hospital and the ¥Sunnybrook Health Sciences Centre, Toronto, Ont.

Background: Management of trauma patients is difficult due to their complexity and acuity. In an effort to improve patient care and reduce morbidity and mortality, the World Health Organization (WHO) developed a trauma care checklist. Local stakeholder input at our institution led to a modified 16-item version that was subsequently piloted. Our study highlights the barriers and challenges associated with implementing this checklist at our hospital. Methods: The checklist was piloted over a 6-month period at St. Michael's Hospital, a level 1 trauma centre in Toronto, Canada. At the end of the pilot phase, individual, semi-structured interviews were held with trauma team leaders and nursing staff regarding their experiences with the checklist. Axial coding was used to create a typology of attitudes and barriers toward the checklist and then vertical coding was used to further explore each identified barrier. Checklist compliance was assessed for the first 7 months. Results: Checklist compliance throughout the pilot phase was $78 \%$. Eight key barriers to implementing the checklist were identified: sense of urgency in critically ill patients, unclear roles, no memory trigger, no one to enforce completion, not understanding its importance or purpose, difficulty finding physicians at the end of resuscitation, staff/trainee changes, and professional hierarchy. Conclusion: The WHO Trauma Care Checklist was a 
well-received tool; however consideration of barriers to implementation and staff adoption must be done for successful integration, with special attention to its use in critically ill patients.

Mesh-mediated fascial traction facilitates the delayed primary closure of the open abdomen: a case report. F.-C. Malo, ${ }^{*}$ N. Deslauriers, ${ }^{+}$M. Gosselin. ${ }^{\dagger}$ From the ${ }^{*}$ University of Sherbrooke, Sherbrooke, Que., and the tCSSS Champlain-Charles-Le Moyne, Affiliated University Centre, Greenfield Park, Que.

Background: The abdominal compartment syndrome is an important concern in patients with abdominal catastrophe. Keep the abdomen open prevents this complication but the failure of closure after more than a week is significant. Methods: The objective of this case presentation is to demonstrate the benefits of mesh-mediated fascial traction combined to open abdomen negative pressure therapy (OANPT) for the delayed closure of the open abdomen of a patient. A 68-yearold woman came in hemorrhagic shock and underwent a laparotomy for bleeding control and hepatic lacerations repair. An OANPT was installed. After 1 week, a polypropylene mesh was sutured to the fascial edges between the visceral protective layer and the thick polyurethane sponge of the OANPT. At the following dressing change, the mesh was cut longitudinally into 2 halves and the visceral protective layer was changed. The 2 parts or the mesh was then tightened medially and sutured in the midline. The thick polyurethane sponge was placed on top of the mesh, and the wound was covered with occlusive selfadhesive thin polyethylene sheets. This technique was performed in all subsequent dressing changes. Results: By gradually tightening the mesh during dressing changes, the abdominal wall was closed by delayed primary closure. Twenty one days were required to achieve it. Conclusion: When the abdomen is left open, fascial edges retract, contributing to the failure of the closure. Mesh-mediated fascial traction seems to prevent their retraction and contributes to the success rate of closure. After 6 days, fascial edges were already retracted, it would probably have been impossible to achieve primary closure without mesh-mediated fascial traction. Finally, this technique is easy and accessible and could help surgeons to close open abdomens.

Impact of acute traumatic coagulopathy: What happens in the first hour? M. Guerreiro, J. Tremoleda, D. Frith, $K$. Brohi. From the Bart's and the London School of Medicine and Dentistry, Queen Mary University, London, UK

Background: Acute traumatic coagulopathy (ATC) occurs rapidly after hypovolemic shock in association with traumatic injury. Understanding what happens during the first hour warrants the best care to trauma patients. In this study we examined the changes occurring during the first hour post-injury in an experimental model of ATC. Methods: Twelve male Wistar rats were submitted to trauma and hemorrhage to a target mean arterial blood pressure of $30 \pm 5 \mathrm{~mm} \mathrm{Hg}$. The animals were kept euthermic and were not resuscitated. Blood samples were taken and analyzed at baseline and terminus ( $\mathrm{t} 0$ and $\mathrm{t} 60$ ). Results: After 60 minutes a coagulopathy signature was present in viscoelastic tests (reduction in clot amplitude at $5 \mathrm{~min}\left[\mathrm{CA}^{\prime}\right]$ ) (time $0 \mathrm{v}$. time
$60=53 \pm 2$ v. $49 \pm 3 \mathrm{~mm}, p<0.01)$ and in maximal clot firmness (MCF) (t0 v. t $60=65 \pm 1 \mathrm{~mm}$ v. $57 \pm 1.2 \mathrm{~mm}, p<0.0002)$. In this model both liver and kidney function were deranged (creatinine: t0 v. $\mathrm{t} 60=30.3 \mu \mathrm{mol} / \mathrm{L}$ v. $50 \mu \mathrm{mol} / \mathrm{L}, p<0.002 ;$ ALT: $\mathrm{t} 0$ v. $\mathrm{t} 60=46 \mathrm{U} / \mathrm{L}$ v. $61 \mathrm{U} / \mathrm{L}, p<0.002)$. There was a global fall in the cellular blood component affecting both white blood cells and red blood cells. Conclusion: The organ function seems to be affected immediately after the trauma-hemorrhagic insult alongside with the coagulation impairment. This model warrants a good tool for further investigation of the identified changes and a possible association with the established coagulopathy.

Measuring injury matters: an indicator for age standardized rate of serious child and youth injury-related hospitalizations. I. Pike, ${ }^{*}$ A. MacPherson. ${ }^{\dagger}$ From the ${ }^{*}$ Children's and Women's Hospital of BC, Vancouver, BC, and TYork University, Toronto, Ont.

Background: Despite continued reinforcement of the importance of reliable indicators for injury, there continues to be a lack of objectivity in the way injury severity is measured in children and youth. Such inconsistency may impair future development of trauma triage and may compromise evaluation of pediatric trauma systems. Routinely gathered data, such as hospitalizations, may be subject to variation from sources other than injury incidence. A need for an indicator to define severe injury, which may be less vulnerable to fluctuations due to changes in care, has been identified. The purpose of this study was to identify ICD-10 codes associated with severe pediatric injuries, and to develop a standardized pediatric severity indicator. Methods: The national Discharge Abstract Database and the comprehensive data set of the Ontario Trauma Registry data sets were analyzed, using the Injury Severity Score and the Survival Risk Ratio scoring methods, to produce a candidate list of diagnoses to define severe pediatric injury. Results: The candidate list was subsequently reviewed and refined by trauma surgeons using a modified Delphi method. Conclusion: The indicator developed using this methodology can be used for the evaluation of trends in severe pediatric trauma, and will be less sensitive to changes in health service policy and practice. This research will help to evaluate pediatric trauma systems and to ultimately advance the quality of pediatric injury care.

Influence of access to an integrated trauma system on length of stay: a population-based cohort study. B.K. Brice Lionel, ${ }^{*}$ L. Moore, ${ }^{*}$ B. Cisse, ${ }^{*}$ G. Bourgeois, ${ }^{+}$ $J$. Lapointe. ${ }^{\dagger}$ From the ${ }^{*}$ Universite Laval, Laval, and the tInstitut national d'excellence en santé et services sociaux, Québec, Que.

Background: Despite prehospital transport protocols and interhospital transfer agreements in an integrated trauma system, up to $20 \%$ of major injuries are still treated in a nondesignated hospital. We aimed to evaluate the influence of access to an integrate trauma system on hospital length of stay (LOS). Methods: Despite prehospital transport protocols and inter-hospital transfer agreements in an integrated trauma system, up to $20 \%$ of major injuries are still treated in a non-designated hospital. We aimed to evaluate the influence of access to an integrate trauma system on hospital length of stay (LOS). Results: We selected 
21158 admissions. Access to trauma centres was $91.8 \%$ for all injuries, and $91 \%, 93 \%, 86 \%$ and $94 \%$, respectively for traumatic brain injury (TBI), abdomen/thorax, orthopaedic and spine injuries. Median LOS was 9 days for all injuries and 8, 9, 11 and 12 days for TBI, abdominal/thoracic, orthopaedic and spine injuries. Overall patients with access to trauma care had a similar LOS to patients treated in nondesignated centres. However patients with TBI had a statistically significant increase of $8 \%$ in LOS $(p=0.002)$. Conclusion: Results suggest that in an integrated, mature trauma system LOS for the small proportion of patients treated in nondesignated centres is similar to that of patients treated in trauma centres, overall. However, more research is needed to explain differences observed for specific types of injuries.

Acute care service - Web-based patient management tool. P. Dust, B. Clapson. From the University of Saskatchewan, Saskatoon, Sask.

Background: Effective communication is the cornerstone of high quality patient care and clinical education. In our Division of Plastic \& Reconstructive Surgery, the structure of the highturnover, multicentred, team-based Acute Care Service (ACS) lends itself to potential errors in communication with the risk of losing track of patients and key patient information. The purpose of this project was to improve communication by developing a Web-based communication system using a project management application. Methods: We tested the Web-based project management applications Google Drive and Trello. As it is more visual and intuitive, we chose to develop Trello for use on the ACS. Results: Trello is a board, list, and card based system. The ACS constitutes the "board," and separate lists exist for inpatients, an orphan list, 2 weeks of trauma OR time, and teaching. In the lists, each patient (or student in the teaching list) becomes a card on which the individual's name and relevant details are recorded. Checklists and key attachments (i.e., photos, videos, journal articles) specific to each card can be created and stored in the system. All changes are automatically recorded, and cards can be colour coded to accommodate rounding preferences. All the information on the cards can be moved between lists with one click as patients are transferred between sites. Once a list or card is finished, it can be deleted or archived for reference/research purposes. Conclusion: Pending implementation, this notional system will improve communication, patient care, and teaching on the ACS.

A randomized, double-blinded, placebo-controlled pilot trial on the efficacy of early enoxaparin: the optimal timing of thromboprophylaxis in traumatic intracranial hemorrhage study. T. Rice, ${ }^{*}$ N. Sne, ${ }^{*}$ M. Meade, ${ }^{*}$ R. Whitlock, ${ }^{*}+$ S. Amenawer, ${ }^{*}$ K. Reddy, ${ }^{*}$ S. Schulman, ${ }^{*}$ D. Jichici, ${ }^{*}$ R. Roberts, ${ }^{*}$ D. Cook. ${ }^{*}$ From *McMaster University, and TPHRI, Hamilton, Ont.

Background: Emerging evidence suggests that enoxaparin may be safely administered 48 hours after traumatic intracranial hemorrhage for venous thromboembolism prophylaxis. We conducted a single-centre, randomized, double-blinded, placebocontrolled pilot trial to assess the feasibility of a larger-scale trial comparing the efficacy of early (48 h) versus delayed (120 h) enoxaparin in this setting. Methods: Patients with traumatic intracranial hemorrhage without progression on a 24 hour repeat head CT scan were randomized to subcutaneous enoxaparin $30 \mathrm{mg}$ twice daily or placebo at 48 hours after admission. At 120 hours after admission, all patients received twice daily open-label enoxaparin. Patients underwent compression ultrasounds within 72 hours of enrolment and then weekly. Primary feasibility outcomes included assessing: recruitment potential; timely randomization; complete study drug administration; and ultrasound compliance. Secondary clinical outcomes were proximal lower extremity deep vein thrombosis (DVT), pulmonary embolism (PE), intracranial hemorrhage progression, and nonintracranial bleeding. This was an internal pilot trial and investigators remain blinded; accordingly, we analyzed the clinical outcomes on the entire cohort of patients. Results: We screened 155 patients over 12 months and randomized 32 patients. Time to first dose was $46.2 \pm 3.5$ hours. Complete study drug administration occurred for $97.7 \%$ of scheduled doses. Time from enrolment to baseline ultrasound was $29.2 \pm 16.8$ hours. Weekly ultrasounds occurred without exception. Clinical outcomes were: 2 (6.3\%) DVTs; 1 (3.1\%) PEs; 2 (6.3\%) major and 3 (9.4\%) minor bleeding episodes; and no intracranial hemorrhage progression. Conclusion: Our pilot trial demonstrates that a larger-scale trial is feasible but requires expansion to a multicentre collaboration.

Clarifying the role of follow-up imaging in blunt splenic injury - experience from a level 1 Canadian trauma centre. D. Paskar, ${ }^{*}$ A. Coates, ${ }^{+}$C. Uy, $^{+}$S. Faidi, ${ }^{+}$N. Parry, ${ }^{\neq}$ B. Petrisor, ${ }^{\dagger}$ M. Bhandari, ${ }^{\xi}$ F. Gastaldo. ${ }^{+}$From *Western University, London, Ont., tHamilton Health Sciences Centre, Hamilton, Ont., fLondon Health Sciences Centre, London, Ont., and §McMaster University, Hamilton, Ont.

Background: Nonoperative management (NOM) of blunt splenic injuries (BSI) is accepted as standard of care in stable patients. An aspect of NOM that continues to be debated is the use of routine re-imaging to detect delayed vascular injury. This study seeks to add to the collective NOM experience and elucidate whether discretional reimaging is safe. Methods: This retrospective (2005-2012) study used data from a centre where BSI reimaging is discretional. All BSI patients managed without abdominal surgery or embolization for the first 36 hours postinjury were included. Data was abstracted regarding demographics, imaging (indications and findings), interventions and clinical outcomes. Initial BSI findings were graded using the American Association for the Surgery of Trauma scale. Results: Repeat inpatient imaging was performed in 101 of 198 (51\%) eligible cases: 79 (40\%) with CT and 22 (11\%) with ultrasound, with median timing of 3 and 4.5 days, respectively, post-injury. Splenic salvage was achieved in 193 (97\%) cases; 5 required delayed splenectomy. A further 5 patients underwent embolization. All cases requiring delayed interventions were identified by repeat CT: 8 indicated by clinical factors such as declining hemoglobin or hemodynamic status; the other 2 studies performed as routine follow-up in initial grade 3 BSI. Conclusion: Excellent outcomes can be achieved without routine reimaging of all BSI. Our data suggest that re-imaging of grade 1 and 2 BSI is only required when clinically indicated, whereas routine reimaging for grade 3 BSI may still be necessary. Formal BSI grading at time of original CT reporting will assist with this decision-making. 
Defining the platelet count that matters in traumatic brain injury patients on antiplatelet therapy. L. Butvidas, P. Rhee, V. Pandit, N. Kulvatunyou, T. O'Keeffe, A. Tang, H. Aziz, G. Vercruysse, R. Friese, B. Joseph. From the University of Arizona, Tucson, Ariz.

Background: Platelet dysfunction has been attributed to progression of initial intracranial hemorrhage (ICH) on repeat head computed tomographic (RHCT) scans in patients on prehospital antiplatelet therapy. However, there is little emphasis on the effect of platelet count and progression of ICH in patients with traumatic brain injury (TBI). The aim of this study was to determine the optimal platelet count for progression on RHCT and neurosurgical intervention in patients on antiplatelet therapy. Methods: We performed a prospective cohort analysis of all TBI patients with an ICH on prehospital antiplatelet therapy. Antiplatelet therapy was defined as ASA, clopidogrel, or combination of both. Admission platelet count was recorded and used for analysis. Receiver operating characteristic (ROC) curves were plotted to identify the optimal platelet count for progression on RHCT and neurosurgical intervention in patients on antiplatelet therapy. Results: A total of 226 patients were enrolled. Patients with progression on RHCT had lower mean platelet count $(153 \pm$ $83 \times 103$ v. $235 \pm 66 \times 103 ; p=0.01)$. Platelet count $\leq 135000$ $(\mathrm{AUC}=0.80)$ and platelet count $\leq 95000(\mathrm{AUC}=0.92)$ were the optimal threshold points for progression on RHCT and neurosurgical intervention, respectively. Patients with platelet count $\leq 135000$ were 16.6 times (95\% CI 6.3-24) more likely to have progression on RHCT and patients with platelet count $\leq 95000$ were 33.9 times (95\% CI 8-139) more likely to require neurosurgical intervention. Conclusion: A platelet count of less than $135000 \mathrm{~mm}^{3}$ in patients on antiplatelet therapy is predictive of both radiographic and clinical worsening. This is a clinically relevant target intended to help tailor and improve management in patients on antiplatelet therapy. Future studies are required to determine whether platelet administration based on platelet count improves outcomes.

Surgical intervention in pediatric trauma at a regional lead trauma hospital. P. Zamiara, ${ }^{*}$ T. Charyk Stewart, ${ }^{+}$ B. Batey, ${ }^{+}$D. Fraser ${ }^{+}{ }^{+}$N. Merritt. ${ }^{+}$From the ${ }^{*}$ Schulich School of Medicine and Dentistry, Western University, London, Ont., and the TLondon Health Sciences Centre, London, Ont.

Background: Injury continues to be a leading cause of mortality and disability in pediatric patients. The implementation of organized trauma systems ensuring access to timely surgical intervention and subject to continuing quality improvement (including review of resource utilization) strives to decrease mortality and morbidity. We aimed to determine operating room utilization rates by the various surgical services, as well as the frequency and predictors of surgical intervention, in the management of pediatric trauma patients at Children's Hospital, London Health Sciences Centre (CH-LHSC). Methods: Pediatric trauma patients treated at CH-LHSC from 2002 to 2013 were analyzed for demographic characteristics, vital signs at presentation to hospital, Injury Severity Score, length of stay (LOS), and outcome. If surgical intervention was needed, the most responsible surgical service was determined. Results: Of 784 patients, 258 (32.9\%) required surgical intervention. These patients were older, more severely injured, had a longer LOS, and were more frequently discharged to another acute care or rehabilitation facility, than patients who did not require operative management. The mortality was not significantly different between these 2 groups. Seventy-four $(28.7 \%)$ of the surgical patients required intervention within 4 hours of admission. Pediatric neurosurgery treated the greatest proportion of these acute patients $(44.6 \%)$, while pediatric orthopedic surgery was most responsible for $40.0 \%$ of all surgical patients. Conclusion: About one-third of pediatric trauma patients require surgical intervention, of who nearly onethird require intervention within 4 hours of admission. Neurosurgery is most often the most responsible service for acute patients, while orthopedic surgery is most used overall.

Validation of hand motion analysis as an objective assessment tool for the fast examination. M. Ziesmann, ${ }^{*}$ J. Park, ${ }^{*}$ B. Unger, ${ }^{*}$ A. Kirkpatrick, ${ }^{+}$A. Vergis, ${ }^{*}$ D. Kirschner, ${ }^{*}$ L. Gillman. ${ }^{*}$ From the *University of Manitoba, Winnipeg, Man., and the TUniversity of Calgary and Foothills Medical Centre, Calgary, Alta.

Background: Point of care ultrasonography (US) is considered a standard part of trauma assessments, but no objective tools to assess proficiency and ensure high-quality exams exists. Hand motion analysis (HMA) has been validated as a measure of surgical skill but has not previously been applied to US. The HMA was assessed for construct validity in Focused Assessment with Sonography for Trauma (FAST) performance. Methods: Two cohorts of 12 expert and 12 novice ultrasonographers performed a FAST examination on a healthy volunteer. Hand motions were recorded at $240 \mathrm{~Hz}$ with the trakSTAR 3D electromagnetic motion-tracking device (Ascension Technology) and the recorded data was analyzed for comparison of the cohorts using a Student $t$ test. Results: Time of exam was not different between cohorts (expert: 345.9 s, novice: $475.7 \mathrm{~s}, p=0.12$ ). Total path length of travel was shorter and number of discreet movements was less in the expert cohort for left hand (18.52 v. $28.01 \mathrm{~m}, p=0.03 ; 109.5 \mathrm{v}$. $193.9, p=0.027)$ and right hand performance $(14.25 \mathrm{v} .32 .09 \mathrm{~m}$, $p<0.01 ; 153.5$ v. $258.5, p=0.03)$ versus the novice cohort. Both total path length travelled and total number of discreet movements were associated with expertise level in logistic regression modeling with areas under the receiver operating curves of 0.8269 and 0.8205 , respectively. Conclusion: Hand motion analysis provides objective, valid metrics that can function as an adjunct measure to identify trainees who have reached competence in FAST sonography. These automated metrics can be incorporated into simulators or credentialing programs to provide feedback to learners to improve future performances.

Platelet transfusion in trauma resuscitation: Early high ratios improve survival in Qatar. R. Peralta, ${ }^{*}$ A. Vijay, ${ }^{*}$ R. Consunji, ${ }^{+}$A. El Menyar, ${ }^{+}$M. Asim, ${ }^{*}$ A. Parchani, ${ }^{*}$ H. Abdulrahman, ${ }^{*}$ A. Zarour, ${ }^{,} H$. Al Thani, ${ }^{\dagger}$ R. Latifi. ${ }^{*}$ From the *Hamad Medical Corporation and the tHamad General Hospital, Doha, Qatar

Background: There is a growing debate surrounding the optimal ratio of platelet to packed red blood cell (PRBC) in massive transfusion protocols (MTP) for patients with traumatic hemorrhage. 
The discussion has evolved to include the time at which the appropriate ratio is achieved. This report aimed to compare the effect of attaining a high (platelet to PRBC $\geq 1: 1.5$; HMTP) or low ratio (platelet to $\mathrm{PRBC} \leq 1: 1.5$; LMTP) MTP within 4 hours on trauma patients receiving MTP. Methods: Data were obtained from the trauma registry of the Hamad Trauma Center in Doha, Qatar. All adult trauma patients who received $\geq 10 \mathrm{U}$ of PRBC over the initial 24 hours post-injury, from Jan. 1, 2010, to Dec. 31, 2012, were included. Patients were classified as HMTP or LMTP. Patients were followed up to 30 days, hospital discharge or death. Outcomes measured were mortality, multi-organ failure (MOF) and infectious complications. Results: Fifty-eight patients met inclusion criteria (HMTP-39 and LMTP-19). Admission hemoglobin level, arterial $\mathrm{pH}$, international normalized ratio, Injury Severity Score and Revised Trauma Score were similar between both groups. HMTP was associated with lower crude mortality $(36.8 \%$ v. $84.6 \%, p=0.001)$ and MOF $(42.1 \% \mathrm{v}$ $87.2 \%, p=0.001)$ when compared with LMTP. The reverse was noted for rates of ventilator associated pneumonia $(38.9 \% \mathrm{v}$. $10.8 \%, p=0.021)$ and wound infection $(50 \% \mathrm{v} 10.8 \%, p=0.002)$ when compared with LMTP. Conclusion: These results suggest that the survival advantage of HMTP for trauma patients is realized early. The aggressive attainment of HMTP within 4 hours post-injury is associated with reduced mortality and MOF rates and increased infectious complications..

Are we transfusing more and operating less in isolated blunt splenic injury with new hemostatic methods? A retrospective study. N. Howatt, S.-Y. Yeh, Y. Leblanc, J. Lampron. From the University of Ottawa, Ottawa, Ont.

Background: The management of patients with isolated blunt splenic (IBS) injury remains an area of controversy partly due to the challenges faced with predicting the individual patient's injury evolution - despite the efforts of grading systems with diagnostic imaging. The decision to operate often heavily relies on clinical evolution rather than initial severity determined by imaging. Furthermore, new trauma hemostatic methods have developed over the last few decades, namely tranexamic acid and splenic artery embolization (SAE), which have altered the course of IBS injury management. The primary objective of this study was to evaluate the need for packed red blood cells (pRBCs) with a nonoperative management (NOM) strategy versus an operative management (OM) group. A secondary objective was to compare management of BSI at our institution over the past 2 decades. Methods: We conducted a retrospective review of a trauma database for patients with an IBS injury who presented at The Ottawa Hospital, a level 1 trauma centre over a 10-year period (April 2002-April 2012). The data reviewed included: patient characteristics, type of intervention, number of pRBCs transfused and clinical outcomes. The 2 groups were then compared with assess the number of pRBCs required in each group as well as the mortality. We also looked at change in treatment over the last 2 decades. Results: We recruited 251 patients, of which 76 met inclusion criteria. 70 were treated with NOM and 6 had a splenectomy. One hundread percent $(n=6)$ of the patients with surgical treatment require blood transfusion, while only $11.4 \%$ in the NOM group. The mortality for OM group is $11.7 \%$ and $1.4 \%$ for NOM group. In the NOM, $21.4 \%(n=15)$ had an $\mathrm{SAE}$, which is much higher than in our previous cohort 10 years ago (1.5\%). Conclusion: A total of 251 patients were recruited, of which 76 met inclusion criteria; 70 were treated with NOM and 6 had a splenectomy. A total of $100 \%(n=6)$ of the patients with surgical treatment require blood transfusion, while only $11.4 \%$ in the NOM group. The mortality for OM group is $11.7 \%$ and $1.4 \%$ for NOM group. In the NOM, $21.4 \%(n=15)$ had a SAE, which is much higher than in our previous cohort 10 years ago $(1.5 \%)$.

A prospective analysis of dose dependent effect of prehospital ASA therapy in patients with traumatic intracranial hemorrhage. D. Meyer, P. Rhee, V. Pandit, N. Kulvatunyou, B. Zangbar, A. Tang, T. O'Keeffe, G. Vercruysse, R. Friese, B. Joseph. From the University of Arizona, Tucson, Ariz.

Background: The dose response of prehospital ASA on progression of traumatic intracranial hemorrhage (ICH) and clinical outcomes is not well described. Our aim was to evaluate the dose dependent effects of ASA on clinical outcomes in traumatic brain injury (TBI) patients. Methods: All TBI patients with ICH on initial head computed tomography (CT) were prospectively enrolled. Patients on low dose $(81 \mathrm{mg})$, high dose $(325 \mathrm{mg})$, and no ASA were matched using propensity score in a 1:1:1 ratio for age, gender, Glasgow Coma Scale (GCS), head Abbreviated Injury Scale (H-AIS) score, Injury Severity Score, platelet transfusion, and type and size of ICH. Primary outcome measures were: worsening on repeat head CT (RHCT) and neurosurgical intervention. Results: A total of 216 patients (no ASA: 72, low dose ASA: 72, high dose ASA: 72) were enrolled with mean age $72.5 \pm 12.7$ years, $64 \%$ males, median GCS 13 $(8-15)$, and median H-AIS 3 (2-4). There was no difference in age $(p=0.8)$, H-AIS $(p=0.7)$, platelet transfusion $(p=0.5)$, and type and size of ICH $(p=0.2)$ between the groups. See the Table. Conclusion: Our study demonstrates a dose dependent effect of prehospital ASA on outcomes in TBI with patients on high dose showing worse outcomes. The use of prehospital high dose $(325 \mathrm{mg}$ ) ASA should warrant a routine RHCT.

\begin{tabular}{|c|c|c|c|c|}
\hline \multirow[b]{2}{*}{ Outcome } & \multicolumn{3}{|c|}{ Group; median (IQR) } & \multirow[b]{2}{*}{$p$ value } \\
\hline & $\begin{array}{c}\text { No ASA, } \\
n=72\end{array}$ & $\begin{array}{c}\text { Low dose, } \\
n=72\end{array}$ & $\begin{array}{l}\text { High dose, } \\
n=72\end{array}$ & \\
\hline $\begin{array}{l}\text { Worsening on } \\
\text { RHCT }\end{array}$ & - & $3.0(0.7-4.3)$ & $8.9(1.7-4.6)$ & 0.001 \\
\hline $\begin{array}{l}\text { Neurosurgical } \\
\text { intervention }\end{array}$ & - & $1.6(0.9-3.6)$ & $2.7(1.3-4.6)$ & 0.01 \\
\hline Mortality & - & $2.1(0.6-5.0)$ & $4.4(1.3-15.0)$ & 0.03 \\
\hline
\end{tabular}

Injury severity measures based on the International Classification of Diseases: a systematic review. M. Gagné, ${ }^{*}$ L. Moore, ${ }^{\dagger}$ M.-J. Sirois, ${ }^{\dagger}$ B.K. Brice Lionel. ${ }^{\dagger}$ From the *Institut national de santé publique, Québec, and the †Université Laval, Laval, Que.

Background: The International Classification of Diseases (ICD) is the only classification system available for population- 
based surveillance activities but does not contain information on injury severity. ICD-based injury severity measure can be derived empirically or mapped, but no single approach has been formally recommended. We performed a systematic review to identify ICD-based injury severity measures and compare their relative performance to predict mortality among patients hospitalized for injury. Methods: Medline, Embase and Global Health were searched from their inception through August 2013. We included observational studies that assessed the accuracy of ICD-based injury severity measures to predict mortality and reported discrimination ability using the area under a receiver operating characteristic curve (AUC). Pooled AUC were estimated under random-effects models. Results: Twentyfive eligible studies reported 65 measures of discrimination on 8 ICD-based injury severity measures. Reported AUC ranged from 0.728 to 0.949 . Sixty of the 65 assessments showed excellent $(0.80 \leq \mathrm{AUC}<0.90)$ or outstanding discrimination (AUC > $0.90)$, but large heterogeneity was observed $\left(\mathrm{I}^{2}\right.$ range $33 \%-$ $99 \%$ ). Pooled AUC was 0.873 (95\% CI 0.865-0.881) for Trauma Mortality Prediction Model (TMPM)-9, 0.870 (95\% CI 0.857-0.883) for ICD-9 Injury Severity Score (ICISS) based on the product of survival proportions, 0.856 (95\% CI $0.839-$ 0.874 ) for ICISS based on the worst single injury and 0.859 (95\% CI 0.850-0.868) for ICDMAP. Conclusion: The TMPM and ICISS based on the product of survival proportions appeared to discriminate mortality slightly better than ICDMAP but observed differences were not statistically significant due to high heterogeneity. These results suggest the need for further research to identify the most appropriate ICD-based severity measure for surveillance activities.

Impact of ATLS guidelines introduction on 6 hours mortality in severe trauma in a busy Italian metropolitan hospital. S. Magnone, M.G.C.F. Coccolini, R. Manfredi, D. Piazzalunga, L. Ansaloni. From the Ospedale Papa Giovanni XXIII, Bergamo, Italy

Background: Advanced Trauma Life Support (ATLS) guidelines in the initial management of trauma patients are widely accepted and implemented. We started applying them with the trauma team in April 2011. Aims of this study are to evaluate changes in mortality in the first 6 hours as well as the length of stay (LOS) in the emergency department (ED). Methods: Retrospective study based on patient's admission for trauma. Study period was from April 2011 to December 2012 and control period was from January 2007 to March 2011. Patients were filtered by first diagnosis (ICD 9-CM), excluding traumatic brain injuries, peripheral lesions and burns and by admission ward, considering only intensive care unit (ICU), general surgery and traumatology. Results: There were 272 patients in the control group (CG) and 176 in the study group (SG). The 2 groups were not different for Injury Severity Score (mean 20.1 v. 19.9, $p=1$ ) but were different for age (46.8 CG v. 54.1 SG, $p=$ $0.0007)$ being older in the SG. Deceased patients were not different for age (mean 47 v. 49 yr). Conclusion: Mortality was significantly better in the SG $(14.2 \%$ v. $5.7 \%, p<0.01)$ as well as LOS in the $\mathrm{ED}(162$ min v. $109, p<0.01)$. We evaluated also mortality in the ED (i.e., patients that were not in the operating room or in the ICU): it was better in the SG $(54.5 \%$ v. $40 \%, p=$ 0.42 , not significant).
The effect of an acute care surgery service on the management of appendicitis. R. Krouchev, V. Trottier, G. Champagne-Parent. From the Université Laval, Laval, Que.

Background: In recent years, the acute care surgery (ACS) model has been proposed as a novel way of providing emergent general surgery treatment. Acute appendicitis is one of the most frequent causes of acute abdomen. It has been shown that timely operative intervention reduces complication and rupture rates in patients with appendicitis. We compared hospital length of stay and outcomes in patients treated by appendectomy before and after implementation of an ACS service. Methods: We prospectively reviewed charts from 84 consecutive patients presenting with appendicitis and treated by appendectomy after July 1, 2011. Key time intervals and outcomes were reviewed for this period after implementation of the ACS service. This cohort was compared with a retrospectively reviewed cohort of 85 patients, treated with the traditional model before July 1, 2011. Results: Complication and rupture rates were similar between the 2 groups. Time to consult ( 1.87 v. $6.81 \mathrm{~h} ; p<0.0001)$, overall time to OR (7.2 v. $11.3 \mathrm{~h} ; p<0.0001)$ and hospital length of stay $(1.7$ v. $2.25 \mathrm{~d} ; p=0.0034)$ were significantly decreased in the ACS model. Statistical significance was even stronger when uncomplicated appendicitis only was compared (1.0 v. $1.9 \mathrm{~d}, p=0.0008)$. Conclusion: An acute care surgery service seems to be an efficient way of reducing hospital length of stay in patients being treated surgically for acute appendicitis.

\section{Risk factors for pulmonary embolism after severe extremity injury: a national trauma data bank analysis. E. Joos, M. Tadlock, K. Inaba, K. Vogt, D. Grabo, $D$. Demetriades, O. Okoye. From the Los Angeles County and USC Medical Center, Los Angeles, Calif.}

Background: Military extremity injuries have a high incidence of pulmonary emboli (PE), most of which are not associated with concomitant deep venous thrombosis (DVT). We sought to determine the incidence of PE after severe civilian extremity injury and its association with DVT. Methods: The National Trauma Data Bank was queried from 2002 to 2009 for patients sustaining long bone fractures requiring internal or external fixation (LBF), extremity amputation or extremity vascular injury requiring repair. The primary outcome was incidence of PE. Demographics, injury data, and outcomes were extracted. Risk factors for PE were identified using multivariable logistic regression. Results: Of the 320963 patients, $96 \%$ sustained blunt trauma. Vascular injury occurred in $0.7 \%$ and amputation in $0.6 \%$. The overall incidence of $\mathrm{PE}$ was $0.6 \%$, while the incidence of DVT was $1.4 \%$. The PE incidence was higher after amputation $(1.3 \%)$ and extremity vascular injury (1.4\%) then after LBF $(0.6 \%)(p<0.001)$. The DVT incidence was higher after extremity vascular injury $(7.6 \%)$ then after extremity amputation $(4.2 \%)$ and LBF $(1.4 \%)(p<0.001)$. Of all patients with PE only $17 \%$ had an identifiable DVT. Independent risk factors for PE after multivariate analysis included DVT (OR 9.36), Injury Severity Score $>25$ (OR 1.64), chest abbreviated injury score (AIS) $>3$ (OR 1.41), lower extremity AIS > 3 (OR 1.53), and pelvic fracture (OR 1.35). Conclusion: Patients with $\mathrm{PE}$ after extremity vascular injury were more likely to have a concomitant DVT identified. In patients with extremity injury requiring long bone fracture fixation, 
amputation or vascular repair, the presence of DVT was an independent factor associated with pulmonary embolism.

Can "time to crossmatch" in the hemorrhaging trauma patient be used as a triage tool for resource allocation during a nationwide blood shortage? L. McMurray, B. Nascimento, G. Cleghorn, H. Tien. From the Sunnybrook Health Sciences Centre, Toronto, Ont.

Background: Canadian Blood Services predicts a nationwide blood shortage in the near future. Hemorrhaging trauma patients use $25 \%$ of the national blood supply; therefore, it is essential to identify predictors of inevitable mortality in this population to make informed decisions regarding resource allocation. A variable that has not been previously considered, and one that is available in $100 \%$ of patients is "time to crossmatch." The purpose of this study is to determine whether "time to crossmatch" is a predictor of mortality, if a cut-off point exists where mortality reaches $100 \%$, and therefore if it could be used as a triage tool during a nationwide blood shortage. Methods: This is a retrospective cohort study (2003-2013) of bleeding trauma patients who do not have significant brain injuries. These were identified using our trauma registry as requiring at least one unit of red cell transfusion, with an Abbreviated Injury Score for the head of $\leq 3$. We analyzed for an association between "time to crossmatch" and mortality. Results: Late time to crossmatch (> $45 \mathrm{~min}$ ) trends toward increased mortality likelihood, however this was not statistically significant. Specifically, compared with the reference group of 0-30 minutes, odds ratio of mortality was 1.7 (95\% CI 0.6-4.7) for the 31-45 minutes group and 2.4 (95\% CI $0.7-7.8)$ for the over 45 minutes group. Conclusion: There is a trend toward an increase in mortality as the "time to crossmatch" increases. However, the relationship was not found to be significant and therefore should not be used to influence the clinical decision of resource allocation during a nationwide blood shortage.

Overutilization of helicopter transport in the minimally injured: a healthcare system problem that should be corrected. G.A. Vercruysse, R. Friese, A. Hashmi, M. Khalil, A. Tang, T. O'Keeffe, N. Kulvatunyou, D. Green, B. Joseph, P.M. Rhee. From the University of Arizona, Tucson, Ariz.

Background: Mortality benefit has been demonstrated for trauma patients transported via helicopter, but at great cost. This study quantifies patients who did not benefit from helicopter transport to our facility and demonstrates potential cost savings when transported instead by ground. Methods: Retrospective review of all trauma patients transported by helicopter to our centre over 7 years (2007-2013) was conducted. Those dead on arrival were excluded. Minimally injured patients were defined as those going neither to operating room nor intensive care unit after workup. Analyzed data included demographics, injury severity parameters, length of stay, mortality, procedures performed, transport cost, and distance from scene. Results: Of 1047 helicopter transports, 981 met inclusion criteria. Median age was 33 years, $68 \%$ male. Seventy-five percent had Injury Severity Score (ISS) under 15 and of those $37 \%$ an ISS under 5. All were hemodynamically stable. Mean distance travelled was $106 \pm 67$ miles. Sisty-three percent were minimally injured. Mortality was $2.3 \%$ with no mortality in the minimally injured.
Median length of stay was 3 days in the overall population, and 1 for the minimally injured. Helicopter transport averaged \$18 000 per patient. Conclusion: Nearly two-thirds of patients transported by helicopter were minimally injured. Revised policies to identify patients who do not benefit from helicopter transport should be developed. Annual savings of over \$1 000000 was identified were minimally injured patients transported by ground. Education to first responders calling for transport and identification of alternate transportation would be both safe and financially beneficial to our system.

A comparative study of cycling injuries: April 1999 to
March 2004 versus April 2008 to March 2013. H. Knight, A. Keenan. From the The Ottawa Hospital, Ottawa, Ont.

Background: Cycling has increasingly become a popular mode of transportation in the city of Ottawa. This study was undertaken to determine whether there has been an increase in major cycling injury admissions over 2 time periods. Methods: A retrospective review of trauma data was performed to compare 2 time periods: April 1999 to March 2004 versus April 2008 to March 2013. Data elements included age, helmet use, documented alcohol level, Injury Severity Score (ISS), length of stay (LOS) and mortality. Patients with ISS of 12 or greater, trauma codes and/or died in emergency were included. Results: The average number of major cycling injuries has more than doubled over the 2 time periods (average $\mathrm{T} 1=11.2 \mathrm{v}$. average $\mathrm{T} 2=24.2$ ). The ISS and LOS have remained stable despite an average age increase of 6.5 years. The use of helmets has increased to $34.8 \%$ from $30 \%$. The average mortality rate has decreased to $8.9 \%$ (T2) from $16 \%$ (T1). The average alcohol use has also decreased to $7.4 \%$ (T2) from $16 \%$ (T1). Conclusion: There has been a significant increase in the number of major cycling injuries over the last 5 years; however, mortality and associated ethanol use have been halved. This may be partially explained by the profile of the cyclists, who now cycle for health, environmental, lifestyle and economic reasons. These cyclists are typically fit and younger than the average trauma patient, which may contribute to their improved outcome.

\section{Exception of written consent for emergency research: the views and beliefs of Canadians. A. Perez, ${ }^{*}$ S. Trpic, ${ }^{+}$ S. Rizoli, ${ }^{+}$B. Nascimento. ${ }^{*}$ From the ${ }^{*}$ Sunnybrook Health Sciences Centre and tSt. Michael's Hospital, Toronto, Ont.}

Background: In the United States, community consultation is commonly used as a requirement for conducting emergency research without informed consent. However, in Canada, it is mostly used in public health research, with low utilization in trauma research. We recently completed a community consultation for a large multicentre trial involving trauma patients; and here reports on the views and beliefs of Ontarians toward emergency research without written informed consent. Methods: In the USA, community consultation is commonly used as a requirement for conducting emergency research without informed consent. However, in Canada, it is mostly used in public health research, with low utilization in trauma research. We recently completed a community consultation for a large multicentre trial involving trauma patients; and here reports on the 
views and beliefs of Ontarians toward emergency research without written informed consent. Results: The survey showed that $75.8 \%$ of the participants found acceptable to be enrolled in a study involving waiver of consent. A multivariate analysis demonstrated a statistically significant difference of men responding "yes" as compared with women. A total of $80.7 \%$ felt that exception of consent in an emergency setting is justified. $85.8 \%$ believe that research is in the best interest of the patient and community. Interestingly, $70.8 \%$ of respondents approved the enrolment of children between 15 and 18 years old in emergency research. Conclusion: The views and beliefs of Ontarians on emergency research with the exception of informed consent are positive, receptive and favourable with a high degree of acceptance.

Impact of a massive transfusion protocol institution at a civilian level 1 trauma centre. C. Malo, D. Bracco, D. Roizblatt, J. Al-Oweis, D. Deckelbaum, P. Fata, B. Bernardin, K. Khwaja, T. Razek. From the McGill University Health Centre, Montréal, Que.

Background: Current evidence suggests that the implementation of a massive transfusion protocol involving a 1:1:1 ratio of blood components can reduce acute coagulopathy and increase survival in bleeding trauma patients. Trauma physicians at our facility began to use a 1:1:1 ratio for massive transfusion in 2006. In 2011, a standardized massive transfusion protocol was implemented at our institution. The main objectives of this study were to evaluate the impact of a massive transfusion protocol (MTP) on door to transfusion delays, on the fresh frozen plasma (FFP)/packed red blood cells (PRBC) and platelets/ $\mathrm{PRBC}$ ratios and on the total number of blood products transfused. Methods: This was a 5-year retrospective analysis of adult bleeding trauma patients. Patients transferred from another institution and patients on anticoagulant therapy were excluded. Data from our trauma database and from the blood bank were cross-referenced and analyzed in 2 groups: the preimplementation group and the post-implementation group. Results: Between January 2008 and March 2013, 154 patients met our inclusion criteria. Both groups were similar for age, sex ratio and Injury Severity Score. Mean delay for first PRBC transfusion was significantly reduced after massive transfusion protocol implementation (71 v. $40 \mathrm{~min} ; p=0.008$ ). Total number of PRBC, FFP, platelets and cryoprecipitates at 1, 6 and 24 hours did not differ significantly between both groups. There was no significant difference before and after MTP institution for average FFP/PRBC and platelets/PRBC ratios. Conclusion: At our institution, the implementation of standardized massive transfusion protocol was with a significant reduction in mean delay for first PRBC transfusion.

Survival advantage of cardioselective $\beta 1$-blockade in sepsis. I. Ibrahim-Zada, P. Rhee, J. Santoro, G. Vercruysse, T. O'Keeffe, R. Friese. From the University of Arizona, Tucson, Ariz.

Background: Utilization of $\beta 1$-adrenoreceptor antagonists (B1AA) in the management of sepsis remains controversial. Recent studies have demonstrated cardioprotective effect of $\mathrm{B} 1 \mathrm{AA}$ in the treatment of sepsis in animals and humans. We hypothesized that B1AA therapy would lead to survival benefits in patients with sepsis. The aim of this study was to describe mortality outcomes in intensive care unit (ICU) patients with sepsis who received B1AA. Methods: We prospectively collected data on patients with sepsis hospitalized in ICU at an urban tertiary referral centre from Jan. 1, 2008, through Mar. 31, 2011. Propensity matching analysis (PMA) was performed to match patients who received B1AA with controls based on source of infection, severity of disease, laboratory results, hemodynamic parameters, and supportive measures. Our primary outcomes were in-hospital mortality and length of hospital stay. Kaplan-Meier analysis was used to identify between-group differences. Results: A total of 222 patients were included in the PMA (111 in each group). A total of $37 \%$ of patients receiving B1AA treatment died compared with $49 \%$ of patients without B1AA $(p=0.0003)$. Median survival in the B1AA group was 34 days versus 15 days in control group $(p=0.009)$. KaplanMeier survival analysis showed that B1AA administration during ICU stay improved in-hospital survival in patients (HR 0.46, 95\% CI $0.3-0.7 ; p<0.0001)$. Conclusion: Use of $\beta 1$-selective blockade during ICU course is beneficial and associated with a 2 -fold mortality reduction in septic patients. Further prospective studies addressing optimal dosing schedules and exploring for potential mechanisms are warranted.

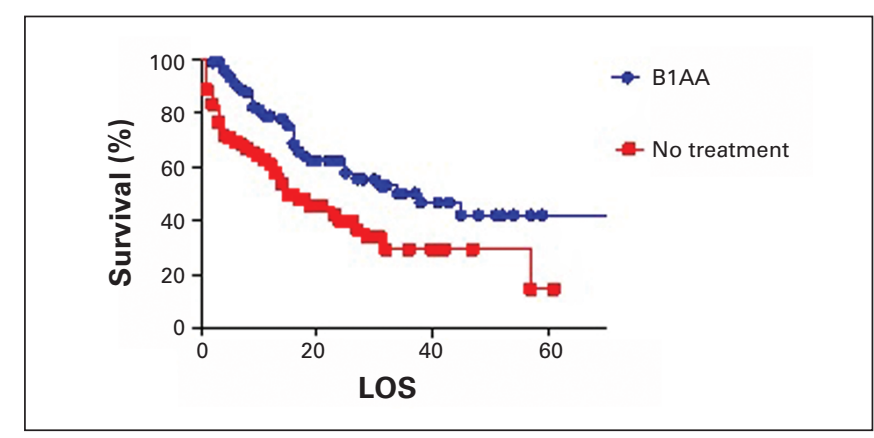

A systematic review on the safety and utility of prehospital blood transfusions. S. Jenneson, ${ }^{* t}$ J. Tallon, ${ }^{*} \neq \S$ E. Vu, ${ }^{*} \neq £ \rrbracket$ R. Gezer. I From the ${ }^{*}$ Department of Emergency Medicine, Vancouver General Hospital, the †British Columbia Emergency Health Services, $¥$ British Columbia Ambulance Service, Provincial Air Evac \& Critical Care Transport Program, the §Faculty of Medicine, University of British Columbia, and the TDepartment of Critical Care Medicine, Vancouver General Hospital, Vancouver, BC

Background: Prehospital fluid resuscitation of trauma patients in shock is well debated in the literature. These patients often require blood transfusions for restoration of oxygen carrying capacity and vital organ perfusion to prevent morbidity and mortality associated with hemorrhagic shock. Observational data supports that patients in hemorrhagic shock require blood transfusion before definitive surgical control. We performed a systematic review on prehospital blood transfusion to determine the safety and utility of this practice. Methods: Using PRISMA methodology, a literature search was performed using Embase and Medline electronic databases, looking for articles on prehospital blood transfusion in trauma patients. Specifically, data was extracted for documented adverse transfusion-related reactions, and indications for transfusion. 
Results: Emergency medical services (EMS) that perform prehospital blood transfusion are rare, and there is a paucity of data on this topic. Reported barriers to prehospital blood transfusion include blood product availability, and capability of EMS to properly store blood products. We found that although similar, the indications for prehospital blood transfusion in trauma patients are not standardized. We did not find evidence of any documented adverse reactions related to prehospital blood transfusion, but there were limitations to this observation. Conclusion: Very few civilian EMS agencies have prehospital blood transfusion programs. The EMS agencies that do transfuse trauma patients feel that it is a safe and justified practice. Due to the number and quality of studies that exist, more research is warranted to quantify the relative ratio of risk to benefit of this practice

A characterization of trauma patients that have undergone PSA. R. Green, ${ }^{*}$ M. Butler. ${ }^{\dagger}$ From *Dalhousie University and the tCapital District Health Authority, Halifax, NS

Background: The trauma patient is a subset of the procedural sedation (PSA) population that is more critical. The normal rigors of PSA may have a pronounced effect on their physiology. This study is a characterization of said population's demographics and incidence of adverse events.. Methods: This is a retrospective analysis of 107 patients that were trauma activations at the Halifax Infirmary (HI). Data were collated from the Nova Scotia Trauma Registry and the PSA registry. We compare these patients to 4223 patients in the general PSA population. Results: A total of $75.3 \%$ of patients were male, and $78.2 \%$ were assessed as ASA I. Patients had a mean age of 42.2 years, and $84.2 \%$ received PSA for orthopedic manipulations. After adjusting for age, ASA and sex, patients in the trauma group were more likely to be hypotensive (OR 1.79, $p=0.023$ ). Almost all trauma patients $(97.0 \%)$ were admitted and had an average length of stay (LOS) of 10.5 days, $9.9 \%$ of patients were admitted to the ICU with a mean LOS of 6.4 days, and the majority of patients $(61.4 \%)$ were admitted to a step-down unit with a mean LOS of 3.02 days. Two trauma patients died in hospital. Conclusion: Patients in the trauma group were more likely to be hypotensive than the general PSA population. A total of $1.9 \%$ of patients in the trauma group died in-hospital, and $71.3 \%$ were admitted to at least a step-down unit. More research is necessary to optimize outcomes of trauma patients undergoing PSA.

Presentation and outcomes in Native Americans after traumatic brain injury. I. Ibrahim-Zada, J. Wynne, B. Zangbar, B. Joseph, R. Friese, P. Rhee. From the University of Arizona, Tucson, Ariz.

Background: The aim of this study was to investigate ethnic differences in presentation and outcomes in patients with traumatic brain injury (TBI). Methods: We performed a retrospective analysis using the State Trauma Registry (STR) from 2008 to 2012. Patients with the diagnosis of TBI were identified and grouped based on ethnicity. Univariate and multivariate regression analyses were performed to explore the relationship between ethnicity, mortality, and length of hospital stay, while controlling for demographics, mechanism, Glasgow Coma Scale, substance abuse, and disposition. Results: The STR data set contained 43514 patients. Of those, American Indian or Alaska Natives comprised 11.5\%. Race combined with age, alcohol, or mechanism was significantly associated with mortality and longer length of stay after TBI. Native American (NA) patients were younger than the state-average (22 v. $39 \mathrm{yr})$. More NA patients had alcohol level above the legal limit $(72 \%)$ and confirmed illegal drug use on admission $(23.7 \% \mathrm{v}$. $16.9 \%, p<0.0001)$. Assaults were the predominant mechanism in NA (48\%): $3.4 \%$ due to domestic abuse, $90.5 \%$ were intentional. Despite higher rate of severe TBI (19\% v. $12.4 \%)$, the overall mortality were lower $(3.5 \%$ v. $4.6 \%, p=0.001)$. However, more patients were discharged to rehabilitation and continuous care $(11.5 \% \mathrm{v}$. 6\%). Conclusion: Analysis of Native American patients with TBI in the 5-year period revealed younger age associated with increased rates of substance abuse. The predominant mechanism of assault correlated with overall lower mortality, but higher rate of transfer to rehabilitation facilities. This paper provides important data to inform use of public health interventions.

Knowledge translation to legislative action for all age helmet use for skiing and snowboarding. L. Fenerty, ${ }^{*}$ G. Thibault-Halman, ${ }^{*}$ J. Young, ${ }^{\dagger}$ J. Heatley, ${ }^{\ddagger}$ S. Walling, ${ }^{*}$ D. Clarke. ${ }^{\mathcal{\delta}}$ From the ${ }^{*}$ Queen Elizabeth II Health Sciences Centre, †Nova Scotia Department of Health and Wellness, ¥Atlantic Collaborative on Injury Prevention, and §Dalhousie University, Halifax, NS

Background: This study represents a longitudinal examination of helmet use in skiers and snowboarders from 2010 to 2013. Baseline and subsequent data comparisons were studied to inform policy makers responsible for prevention strategies for skiers and snowboarders. Methods: A mixed methods approach of helmet observations and interviews were completed in 2010. These data led to focus group examination of non-helmet wearers, which influenced the development of the "Helmet Hair" marketing campaign in 2011. The 2012 helmet study results informed legislative decisions and the design of the "Helmet Match" marketing and education campaign to gear up for helmet law enactment and enforcement in 2013. Results: Data from 2010 revealed sex differences for helmet use: $80 \%$ females and $70 \%$ males $(p<0.001)$. Helmet use decreased with increasing age $(p<0.001)$ : helmet use in children was $96 \%$ (2010) versus $100 \%$ (2012) and helmet use in adults was 60\% (2010) versus 83\% (2012). Overall, helmet use went from 74\% (2010) to 90\% (2012) during ongoing education and social marketing campaigns and to $100 \%$ in 2013. Conclusion: In order to fully achieve widespread all-age helmet use, we implemented a multipronged, staged approach of education, legislation and enforcement. This series of helmet studies examines the progression of research evidence translated into legislative change supporting improved health and safety for skiers and snowboarders.

Improving compliance of trauma team leaders' use of documentation tools. E. Clark, M. Waggott, A. Keenan, H. Knight. From The Ottawa Hospital, Ottawa, Ont.

Background: What's your success rate in getting trauma team leaders (TTLs) to document their assessments? On average, TTLs at our Canadian level 1 trauma centre documented a trauma team leader note for $61 \%$ of trauma codes, and completed a pre-printed trauma order sheet $4 \%$ of the time. Past 
research has shown that formal review and feedback can impact performance and behaviour. Methods: A quality improvement strategy was implemented in order to improve documentation. Results: In Q1 documentation rates were 61\% for TTL notes and $4 \%$ for completion of the trauma order sheet. Each quarter there was an increase in documentation compliance and ultimately, by Q6, rates had increased to an impressive 100\% dictated TTL note, and $30 \%$ use of trauma order sheet. The implementation of an automated dictation system and trauma assessment template may also have contributed to the outcome. Conclusion: Low rates of completion of trauma code documentation tools by trauma team leaders at a level 1 trauma centre in Canada increased significantly after initiation of a quality improvement strategy involving quarterly reviews and feedback to physicians regarding their compliance and comparison to peers.

Factors affecting transport time to tertiary care after traumatic brain injury. G. Thibault-Halman, ${ }^{*}$ L. Fenerty, ${ }^{*}$ S. Ackroyd-Stolarz, ${ }^{\dagger}$ S. Karim, ${ }^{\ddagger}$ B. Sealy, ${ }^{\dagger}$ J. Tallon, ${ }^{\neq}$ D. Clarke. ${ }^{*}$ From ${ }^{*}$ Queen Elizabeth II Health Sciences Centre, +Nova Scotia Department of Health and Wellness, and ¥Dalhousie University, Halifax, NS

Background: Neurosurgical care in Nova Scotia is centralized at the Queen Elizabeth II Health Sciences Centre (QEII HSC) Halifax. Transportation is coordinated via a Provincial Trauma Hotline. Using prospectively collected data from the Nova Scotia Trauma Registry and the Emergency Health Services Communications and Dispatch Centre Database, factors such as demographics, mechanism of injury, vitals and computed tomography (CT) at referring facility were examined for their influence on time to hotline call and to access of tertiary care. Methods: Inclusion criteria were a head abbreviated injury score 3 as well as treatment at one or more referring facilities before arrival at the QEII HSC. Exclusion criteria were presentation at the referring facility more than 24 hours after injury and arrival at the QEII HSC more than 24 hours after arrival at the first facility. Patients with chronic subdural hematoma and those injured outside of Nova Scotia were also excluded. Results: A call to the Trauma Hotline was less likely to occur in a timely fashion (within the first hour at the referring facility, $p=0.000$ ), and patients were less likely to have timely access to care $(p=0.000)$ if a CT was performed at the referring facility. Other factors found to be significant were the place of injury (outside the health district; $p=0.049$ ), or higher scene GCS $(13-15 ; p=0.002)$. Conclusion: Several factors were identified as being associated with delay to tertiary care. A highly significant and potentially modifiable factor was CT at a referring facility.

Multimodal rib fracture treatment protocol decreases morbidity in trauma patients. D. Carver, A. Keenan, J. Lampron, A. Lui, G. Pagliarello, J. Hooper, J.-D. Yelle. From The Ottawa Hospital, Ottawa, Ont.

Background: Rib fractures are a significant source of morbidity and mortality among trauma patients. Several therapies have been shown to improve clinical outcomes in this population; however, the ideal treatment modality remains unclear. In 2012,
The Ottawa Hospital, a level 1 trauma centre, implemented a multimodal rib fracture protocol designed to optimize pain control, which is a key component in the treatment of rib fractures. The objective of this study was to measure the efficacy of this protocol. Methods: A retrospective cohort study of a singlehospital trauma registry reviewed all trauma patients with rib fractures admitted between June 2010 to June 2011 (control group, $n=241$ ) and following implementation of a multimodal protocol from June, 2012 to June, 2013 (study group, $n=194$ ). Groups were compared for rates of pneumonia, length of stay (LOS) and mortality. Results: The control and study groups were similar in terms of age (56.0 v. 54.4), Injury Severity Score (23.6 v. 23.6) and number of rib fractures (4 v. 4). The study group demonstrated a lower rate of pneumonia $(5.7 \%$ v. $12.5 \%$; $p=0.02)$ and shorter intensive care unit (ICU) LOS (10 v. $6 \mathrm{~d}$; $p=0.046)$. There was no significant difference in total LOS $(9 \mathrm{~d}$ for both groups, $p=0.53$ ) or mortality (study $7.7 \% \mathrm{v}$. control $6.2 \% ; p=0.54)$. Conclusion: Implementation of a multimodal rib fracture treatment protocol improves patient outcomes by decreasing the rate of pneumonia and patient ICU length of stay but does not improve mortality.

ETOH and falls in the elderly. B. Mews, A. Keenan, H. Knight. From The Ottawa Hospital, Ottawa, Ont.

Background: Falls are the leading cause of injury-related admission for Canadians 65 years of age and older. The mechanism of falls varies but the highest rate was slips and trips at $43 \%$. Little information on the influence of ethanol (ETOH) is available. Methods: A retrospective review and comparison of trauma registry data from a level 1 trauma centre was performed over 2 time periods. Data from 2003 to 2008 (time A) was compared with data from 2009 to 2013 (time B). The data collection elements obtained were; total number of cases, total number of these patients tested for ETOH, number of positive samples, gender and age. Results: Time A revealed 157 cases per year on average. $6.4 \%$ had an ETOH level drawn. On average $53 \%$ of these samples tested positive for ETOH. In time B there were an average 216 cases per year. An ETOH level was drawn on $9.3 \%$ of those patients. On average $51.4 \%$ of these samples tested positive for ETOH. Conclusion: Most trauma centres consistently measure ETOH levels in MVCs; however, ETOH levels in falls are not routinely taken. Only $7.85 \%$ of patients in our study had blood alcohol levels measured. Measurements appear to be associated with a high index of suspicion, as greater than $50 \%$ were positive. In an effort to implement injury prevention initiatives related to falls and to understand the reasons contributing to the frequency of falls in this population, blood alcohol levels should be consistently measured in elderly patients with falls.

Final results of a multicentre prospective cohort study of patient experiences with acute and post-acute injury care. N. Bobrovitz, H.T. Stelfox, J. Kortbeek, M.J. Santana, T. Kline. From the University of Calgary, Calgary, Alta.

Background: To deliver injury care that meets the needs of patients we must incorporate patient perspectives into quality measurement and improvement. Methods: We conducted a prospective cohort study of consecutive injury patients admitted 
to 3 trauma centres in Alberta to obtain patient assessments of injury care. Patients/surrogates were approached before hospital discharge and invited to participate in a 2-part survey study using validated Likert-type scales inquiring about experiences with acute injury care (self-complete survey administered within $72 \mathrm{~h}$ of discharge) and post-acute care (telephone survey 2-3 mo post-discharge). Results: We approached 512 participants, of whom $400(78 \%)$ consented to participate and completed the acute care survey. The post-acute survey was completed by 200 patients/surrogates. Participants were primarily male (70\%) with major injuries (median Injury Severity Score: 20, IQR 16, 27). Among participants who have completed both surveys, the majority rated the overall quality of acute care as high; however, multivariate analysis showed that post-acute care received significantly fewer high quality ratings $(62 \%$ v. $46 \% ; p=0.02)$. The items receiving the most high quality ratings were "injury explanations" (93\%) and "transfer explanations" (96\%) in-hospital, while those receiving the fewest high quality ratings were "information consistency" (36\%) and "pain control" (49\%) inhospital and "guidance through recovery" (40\%) post-discharge. Conclusion: We implemented the Quality of Trauma Care Patient Reported Experience Measure and showed that patients/surrogates give lower quality ratings to post-acute care compared with acute care and that there are opportunities to improve the consistency of information given to patients, pain control, and post-discharge recovery guidance.

Urine trouble: missed ureter injuries. N. Thiessen, K. Kaups, L. Sue. From the University of California, San Francisco at Fresno, Fresno, Calif.

Background: Ureteral injuries due to trauma are uncommon occurring in less than $1 \%$ of all patients. The incidence of missed ureter injuries has been reported to be $38 \%$ with greater morbidity in these patients with delayed diagnosis. We hypothesized that risk factors for missed injury could be described. Methods: The trauma registry for a level 1 trauma centre was retrospectively reviewed for ureter injuries from 1999 to 2013. Patient demographics, mechanism of injury, location and type of ureter injury, time to diagnosis, Injury Severity Score (ISS), associated injuries, operative procedures, length of stay, complications, and outcomes were identified. Results: A total of 1121 patients were identified with genitourinary trauma (GU) out of 24928 trauma patients. 27 ureteral injuries were identified $(2.4 \%$ incidence in GU trauma and $0.1 \%$ for all trauma patients). There were 7 (26\%) missed ureteral injuries. Missed ureteral trauma patients had a significantly $(p<0.05)$ lower ISS (12 v. 17.5) and longer hospital stay $(10.3$ v. 25.3$)$ than nonmissed ureteral injuries. The average time to missed injury diagnosis was 4 days. Eigthy-six percent had associated injuries. All ureter injuries were repaired over a double $\mathrm{J}$ stent by trauma surgeons. The most common complication was urinary tract infection. Conclusion: Ureteral injuries are uncommon and missed ureteral injuries are common. The most common factor for missing an injury was the failure to adequately expose the ureter regardless of associated injuries. Trauma surgeons can appropriately manage ureteral injuries but a high index of suspicion and careful exploration are essential.

Emergency physicians' knowledge of massive transfusion protocol, tranexamic acid indications and vitamin $\mathrm{K}$ antagonist reversal medications in bleeding trauma patients. C. Malo, B. Bernardin, D. Bracco, D. Roizblatt, J. Al-Oweis, D. Deckelbaum, K. Khwaja, P. Fata, T. Razek. From McGill University Health Centre, Montréal, Que.

Background: Since emergency physicians in Quebec are first hand in treatment of trauma patients, it is vital for them to understand how to replace blood loss and when and how to use prothrombin complex concentrate and or tranexamic acid. The objective of this study is to assess Quebec's emergency physicians knowledge of these new therapies. Methods: We created a multiple choice questionnaire. After being face validated by 2 trauma team leaders and 1 emergency physician working at a level 1 trauma centre, the final version included 19 questions. The questionnaire was administered to Quebec's emergency physicians at a regional meeting in November 2013. Results: We administered 182 questionnaires and 144 (79.0\%) were completed. $67.3 \%$ of our responders had either 5 years or less of practice or were residents. Comparable proportions of physicians were working at a level 1, level 2 or level 3 trauma centre. Only $8.4 \%$ correctly defined massive transfusion and $43.6 \%$ did not know what is the ratio of packed red blood cells:fresh frozen plasma (FFP):platelets for which their protocol is aiming. One out of 5 emergency physicians uses FFP for vitamin $\mathrm{K}$ antagonist coagulopathy reversal in a bleeding trauma patient. A total of $27.3 \%$ need approval from an hematologist or another specialist before using prothrombin complex concentrate; $76.5 \%$ don't have a protocol for tranexamic acid and $66.7 \%$ did not know the dosage used in the CRASH-2 trial. Conclusion: Regional guidelines are needed for massive transfusion protocol, prothrombin complex concentrate and tranexamic acid use in bleeding trauma patients.

Cavitary surgery performed outside the operating room - An exercise in futility or a heroic endeavour? K. Vogt, ${ }^{*}$ K. Inaba, ${ }^{*}$ E. Joos, ${ }^{*}$ B. Moffat, ${ }^{\dagger}$ D. Skiada, ${ }^{*}$ L. Lam, ${ }^{*}$ E. Benjamin, ${ }^{*}$ D. Demetriades ${ }^{*}$ From *Los Angeles County and University of Southern California, Los Angeles, Calif., and tWestern University, London, Ont.

Background: Questions have been raised as to the utility of surgical procedures performed outside the operating room (OR). This study was undertaken to describe the epidemiology of cavitary surgery performed outside the OR in patients sustaining severe trauma. Methods: This retrospective cohort study included all trauma patients assessed at Los Angeles County/University of Southern California from 2007 to 2012. All patients who received a thoracotomy or a laparotomy outside the OR were identified. Demographics, injury data, pre-intervention vital signs, and outcomes were identified using the trauma registry. Potential predictors of survival were assessed. Results: A total of 117 patients required cavitary surgery outside the OR. The majority incurred penetrating trauma $(n=62,53 \%)$ and the mean Injury Severity Score was 28 (SD 18). Prior to intervention the mean systolic blood pressure was $65 \mathrm{~mm} \mathrm{Hg}$ (SD 66). The majority of patients $(n=104$, $89 \%$ ) had a thoracotomy in the emergency department (ED), followed by laparotomy in the intensive care unit (ICU) $(n=8,7 \%)$, thoracotomy in the ICU $(n=5,4 \%)$, and laparotomy in the ED $(n=4,3 \%)$. Overall mortality was $86 \%$. When patients receiving ED thoracotomy were excluded, preprocedure SBP was higher (mean 112 , SD 32; $p<0.01)$ and mortality was lower $(50 \%, p<0.01)$. No 
demographic or physiologic variables were associated with survival, and each procedure type and location produced some survivors. Conclusion: To our knowledge, this is the first study to describe the epidemiology of cavitary surgery performed outside the OR. Despite the severity of injury, many will survive, and these surgical interventions are therefore reasonable to perform.

Clinical outcomes in elderly patients suffering from acute subdural hematoma while on preinjury antithrombotic therapy. P. von Marschall, C. Gilbert. From Carilion Clinic, Roanoke, Va.

Background: Compared with young patients suffering acute subdural hematoma (aSDH) from significant head trauma, elderly patients are more likely to tear cerebral bridging veins from minor mechanisms of injury due to physiologic brain atrophy. Therefore, damage to underlying brain structures is less likely, with higher chance of good neurologic outcome. However, many older patients are on chronic antithrombotic therapy, potentially placing them at greater risk for poor outcomes after minor trauma. Methods: A retrospective chart review was performed on patients admitted to the neuro/trauma intensive care unit (ICU) at a level 1 trauma centre between January 2011 and December 2012. Criteria for inclusion were age of 65 years or over and admission diagnosis of aSDH. JMP9 statistical software was used to analyze data. Results: There were 152 patients who met inclusion criteria, with a mean age of 81 and $53 \%$ female. No statistically significant difference was found between treatment subgroups (ASA, warfarin, clopidogrel) and control in terms of initial Glasgow Coma Scale, surgical intervention, reoperation, total and ICU length of stay, mortality, and change in level of care from admission to discharge. Conclusion: The presence of preinjury antithrombotic therapy results in a high likelihood of functional decline in elderly patients suffering aSDH. Remarkably, hospital course and outcomes were not significantly different between patients in the treatment groups and control. Also of note, patients on ASA alone are found to have an equally poor outcome as those on warfarin and clopidogrel, 2 medications with known potential to increase the risk of hemorrhage.

The meaning of ribs fracture revisited. J.-D. Yelle, ${ }^{*}$ A. Keenan, ${ }^{*}$ H. Knight, ${ }^{*}$ K. Yelle, ${ }^{\dagger}$ J. Lampron, ${ }^{*}$ G. Pagliarello, ${ }^{*}$ V. Cranford. ${ }^{*}$ From *The Ottawa Hospital, Ottawa, Ont., and the tUniversity of Ottawa, Ottawa, Ont.

Background: Are ribs fractures (RFs) in an active and aging population an indicator of trauma? Significant proportions of the elderly are admitted with rib fracture. Morbidity increases with age and the number of RFs. The purpose of this paper is to review how RF affects our pa tient population. Methods: Between April 2009 and March 2013, all patients admitted to The Ottawa Hospital (TOH) had data collected prospectively in a trauma registry. This study retrospectively reviewed patients demographics, etiology, number of RF, chest injuries, associated other injuries, rate of pneumonia, intensive care unit (ICU) and hospital length of stay (LOS), the Injury Severity Score (ISS) and mortality. Results: A total of 2736 patients were admitted to TOH with trauma. A total of 743 (27\%) had at least one RF; $69 \%$ were male. The age average was 55 and average ISS was 23 . The most common cause of injuries were falls $(n=300)$, motor vehicle collisions $(n=197)$, motorcycle collisions $(n=65)$, and motor vehicle versus pedestrian incidents $(n=52)$. The average number of $\mathrm{RF}$ was 4 . The average number of RF for patients requiring ICU admission was 6.5. Associated chest injuries were pneumothorax (25\%), hemothorax (8.5\%), pneumo-hemothorax (20\%), flail chest $(13.5 \%)$ lung contusion (37\%), bilateral RF (20\%), associated abdominal injuries (20\%), orthopedic injuries $(38.5 \%)$, spinal injuries $(24 \%)$ head injuries $(20 \%)$ and pelvic injuries (21\%). Only $23(3.1 \%)$ patients had an isolated RF with no other injury found. 216 (29\%) patients were admitted to the ICU for an average LOS of 12 days. Overall hospital average LOS was 18 days. The pneumonia rate was $13 \%$. The mortality rate was $7 \%$ $(n=61)$, with $60 \%$ of all mortalities over the age of 65 . Conclusion: Rib fractures is an important marker of chest trauma and morbidity. RF should be an indication of trauma and should prompt active investigation for other injuries. RF of 6 and higher warrant an admission to ICU for closer monitoring to improve outcome, especially in the geriatric population.

Incidence of pulmonary embolism after traumatic extremity amputation. E. Joos. From Los Angeles County and University of Southern California, Los Angeles, Calif.

Background: The incidence of pulmonary embolism (PE) after trauma is less than $1 \%$. A recent analysis of 103 combat casualties with trauma-associated amputations found a high rate of postinjury PE. Our goal was to determine the incidence of PE and deep venous thrombosis (DVT) after acute traumatic extremity amputation in civilians. Methods: All patients who underwent an acute upper or lower traumatic limb amputation from January 2000 to December 2010 at our level 1 trauma centre were retrospectively identified. Medical records and imaging were reviewed. The primary outcomes were PE and DVT. Demographic and outcome data were extracted. Results: Eighty-seven patients were identified during the study period. The mean age was 40.2 years $( \pm 1.6)$; $78.2 \%$ were male and $10.3 \%$ had an upper extremity amputation. The majority $(86.2 \%)$ suffered a blunt injury mechanism. The mean Injury Severity Score (ISS) was $16.7 \pm 1$ and $23 \%$ had an ISS over 25 . The mortality rate was $1.1 \%$ and the ICU length of stay (LOS) and hospital LOS were $8.3( \pm 1.6)$ and $30.2( \pm 4.1)$ days respectively. In the cohort, $56.3 \%$ received chemical DVT prophylaxis. A chest CT was performed in 19 (21.8\%) and a duplex ultrasound in $8(9.2 \%)$; no PE and 1 DVT $(1.1 \%)$ were identified. Conclusion: No PE and only 1 symptomatic DVT were identified after acute traumatic amputation. The incidence of symptomatic $\mathrm{PE}$ after trauma-associated amputation appears to be significantly lower in the civilian population than in the military population. Further research is warranted to examine the differences between civilian and combat related acute traumatic amputations.

Exposure to major trauma management of learners training to become emergency physicians in New Brunswick. D. Lukanovic, ${ }^{*}$ J. French. ${ }^{\dagger}$ From *Dalhousie University, Halifax, NS, and tDalhousie University, Halifax, NS, Saint John Regional Hospital, NB and New Brunswick Trauma Program, Saint John Regional Hospital, Saint John, NB

Background: The management of a major trauma is challenging and is the subject of ongoing scrutiny at provincial and national 
levels. Learning curves theory suggests that proficiency improves as competency use increases. Without frequent use, competency can be lost as "skill fade" occurs. This may be reflected in data suggesting that increasing departmental exposure to major trauma improves major trauma outcomes. Using data from New Brunswick (NB) emergency departments and Trauma New Brunswick (TNB), the objective of this project was to determine whether medical clerks and emergency medicine residents are receiving enough trauma exposure to become proficient in the management of major trauma patients. Methods: A data extract from TNB was used to determine the count of major trauma cases (defined as patients with an Injury Severity Score of 15) presenting to emergency departments at the Saint John Regional Hospital (SJRH) and The Moncton Hospital (TMH) in 2011 and 2012. The total number of emergency department visits at all emergency departments in NB was also obtained. The proportion of Major Trauma attendances for SJRH and TMH was calculated. This proportion was then used to estimate the count of cases of major trauma presenting to all other emergency departments in NB that do not collect injury scored data. The average exposure to major trauma cases for residents and medical students was then estimated using typical schedules for both groups. Results: Emergency medicine trainees will see approximately 14 cases of major trauma over their entire medical school and residency training in NB. An emergency room physician working a typical schedule of 48 -hour shifts per week will between 5 and 22 cases per year of major trauma depending on the hospital he or she works at. Given the methodological limitations of this study, these are likely to be overestimates. Conclusion: The current literature in medical education suggests that only seeing 14 cases of major trauma over the course of medical training may not be sufficient for an emergency physician to become proficient in major trauma management. This exposure also suggests that maintaining competency may be challenging. Existing training could be augmented with simulation to help learners become skilled at trauma management. Departmental systems should be designed assuming that users may provide care to these critically ill patients relatively infrequently, which would support the use of checklist, refresher tasks and maximizing learning through thorough case review.

A review of ATV injuries in Nova Scotia. M. Butler, ${ }^{*}$ M. Havenga, ${ }^{*} N$. Yanchar. ${ }^{\dagger}$ From ${ }^{*}$ Capital Health District Authority and the tIWK Health Centre, Halifax, NS

Background: All-terrain vehicle (ATV) use in Nova Scotia is a cultural phenomenon that is not regulated in the same way as other motorized vehicles. If ATV use is associated with increased injury severity, it would emphasize the need to study the problem comprehensively. Methods: This is a retrospective of 2928 injuries captured by the Canadian Hospitals Injury Reporting and Prevention Program at the IWK Health Centre over the course of Jan 13, 2013, to June 30, 2013. We compare patients who had injuries related to ATV use with those who did not. All testing was done at a significance level of 0.05 , using Fisher's test to compare categorical variables with the R software package (version 3.01). Results: Overall, there were 10 injuries related to ATVs in this time period. The ATV group was exclusively male with a mean age of 11.6 years. The nonATV group was $55.3 \%$ male with a mean age of 8.38 years. Patients in the ATV group were more likely to be assessed as a Canadian Triage and Acuity Scale score of 3 or over (OR 7.528, 95\% CI 1.71, $45.28 ; p=0.003)$, and more likely to be admitted for treatment (OR
$11.92,95 \%$ CI $1.96,53.17 ; p=0.004)$. Patients in the ATV group were also more likely to have a fracture, as opposed to more minor injuries (OR 4.55, 95\% CI 1.04, 19.86; $p=0.022$ ). Conclusion: Patients in the ATV group had more severe injuries, both prospectively (CTAS) and retrospectively (likelihood of admission). Given the increased injury severity associated with ATV use in Nova Scotia, further research is justified, perhaps at the national level.

Point of care ultrasound utilization and training among trauma providers across Canada: cross-sectional study. E. Aleassa, ${ }^{*}$ M. Ziesmann, ${ }^{*}$ A. Kirkpatrick, ${ }^{+}$C. Wurster, ${ }^{\ddagger}$ L. Gillman. ${ }^{*}$ From the *University of Manitoba, Winnipeg, Man., tFoothills Medical Centre and University of Calgary, Calgary, Alta., and the ¥University of British Columbia, Vancouver, BC

Background: Point of care ultrasound (POCUS) is revolutionizing care of critically ill patients. However, training in POCUS is extremely variable with no clearly accepted or standardized curriculum or certification process. We aim to delineate the training experience and utilization of POCUS among trauma providers across Canada. Methods: A secured e-questionnaire was sent to physician members of the Trauma Association of Canada. The questionnaire included demographics, first exposure and utilization pattern of ultrasound, opinion on current ultrasound training and certification processes and proposed optimum course design. Results: Seventy-two physicians completed the questionnaire (54.1\% response rate, $n=133)$. Among them 38 were emergency physicians, 31 surgeons and 3 physicians from other specialties. Sixty $(83.3 \%)$ used POCUS for purposes beyond focused assessment with sonography for trauma. Twenty-five (34.7\%) were in practice when first exposed to POCUS. Fifty-three $(73.6 \%)$ received formal ultrasound training through a variety of courses. While only $25(34.7 \%)$ underwent a formal certification process, $76.5 \%$ (52) supported changes to the current certification processes. Sixty-five $(92.8 \%)$ believe that residency programs for all trauma providers must include ultrasound training. Conclusion: There is a wide variation in POCUS training among trauma providers in Canada with only a minority completing a formal certification process. There is no consensus on the optimal curriculum for POCUS training yet most providers support early introduction of structured training during residency and believe modifications to the current certification process are necessary.

Evaluation of the transfer delays in trauma patients from an urban hospital ED to the designated tertiary trauma centre. B. Bruckert, P. Barriga. From Santa Cabrini Hospital, Montréal, Que.

Background: The purpose of this study was to identify how we could improve the time for transfer of traumatic brain injury (TBI) patients by evaluating each step of the process. Methods: A retrospective case series was performed involving 40 randomly chosen adult TBI cases between 2010 and 2013, with an initial Glasgow Coma Scale (GCS) score of 7 to 15 requiring transfer. The sequential times for each step were compiled from the triage until the departure of the patient by ambulance. Results: The most common GCS was 15 (27 cases). The median time between arrival at triage and departure by ambulance was 3 hours and 18 minutes. For cases with a presenting GCS score under 14 or involving a dangerous 
mechanism the transfer time was slightly shorter at 3 hours and 6 minutes. The 2 longest steps were: the time required for scan execution and the time elapsed before viewing of the scan by the doctor. Conclusion: Overall the time required to transfer our trauma cases is highly variable. A closer look reveals that the process by which a scan is executed and then viewed does not consistently reflect the urgency. Several elements contribute to the delays, including the absence of an automatic process by which the doctor is advised that the scan is completed. Other avoidable delays occur in cases with a low initial GCS, which should be stabilized and transferred immediately without a scan when the notion of trauma is clear.

Multiple rib fracture care improvements: protocol driven and trauma nurse coordinator case managed. L. Whitman, A. Vukusic, H. Wilson, L. Gerein, Z. Rurka. From the Royal Inland Hospital, Interior Health, Kamloops, BC

Background: Multiple rib fractures (MRF) are associated with significant pulmonary injury and complications. Adequate analgesia in MRF patients is essential in limiting morbidity. This study sought to measure the effects of implementing an organized multimodal MRF care protocol administered by a trauma nurse coordinator (TNC) in a case management role. Methods: This retrospective matched case-control study assessed patients at a level 2 trauma centre with a primary or secondary diagnosis of MRF over a 5-year period, August 2008 to June 2013, before and after implementation of the MRF protocol. Primary outcome measures were length of stay (LOS) and unexpected/secondary admissions to intensive care or step down units. Results: See the Table. Conclusion: The introduction of a formal care protocol with a $\mathrm{TNC}$ in a case management role improves outcomes for patients with multiple rib fractures. Further research may examine which elements of care have the most effect.

\begin{tabular}{|c|c|c|}
\hline \multirow[b]{2}{*}{ Characteristic } & \multicolumn{2}{|c|}{ Group; median (IQR)* } \\
\hline & $\begin{array}{l}\text { Pre-implementation, } \\
\qquad n=15\end{array}$ & $\begin{array}{l}\text { Post-implementation, } \\
\qquad n=15\end{array}$ \\
\hline Age, yr & $66(53-77)$ & $57(53-65) ; p=0.12 \dagger$ \\
\hline $\begin{array}{l}\text { Multiple rib fractures } \\
\text { score }\end{array}$ & $18(9-25)$ & $14(13-17) ; p=0.31 \dagger$ \\
\hline Injury Severity Score & $24(21-27)$ & $21(17-25) ; p=0.13 \dagger$ \\
\hline Length of stay, $d$ & $11(8-17)$ & $8(5-11) ; p=0.027 \dagger$ \\
\hline $\begin{array}{l}\text { Unexpected ICU/SDU } \\
\text { admission, no. (\%) }\end{array}$ & $6(40)$ & $0(0) ; p=0.006 \ddagger$ \\
\hline \multicolumn{3}{|c|}{$\begin{array}{l}\text { ICU = intensive care unit; } \text { SDU = step down unit. } \\
\text { *Unless otherwise indicated. } \\
\text { †Mann-Whitney } U \text {. } \\
\neq \chi^{2} \text {. }\end{array}$} \\
\hline
\end{tabular}

Communication of critical clinical data during inter-hospital transfer of major trauma patients in BC. N. Bradley, N. Garraway, N. Lakha, R. Simons, M. Hameed. From Trauma Services, Vancouver General Hospital, Vancouver, BC

Background: Each year, approximately 500 major trauma cases occur in British Columbia. Almost half require transfer to BC's only level 1 trauma centre, Vancouver General Hospital (VGH), for definitive care. There is no standardized process for trauma patient transfers in BC. A standardized handover process can improve patient safety. Our objective was to assess accuracy and quality of critical clinical data communication during interhospital trauma patient transfer. Methods: A retrospective patient chart audit was conducted for a sample of major trauma patients (Injury Severity Score $\geq 16$ ) transferred to VGH from April 2011 to March $2012(n=243)$. Communication of critical clinical data (Advanced Trauma Life Support variables, laboratory investigations, transfer summary) was assessed objectively and subjectively. Results: Communication via documentation for major trauma patients requiring transfer to VGH for management was suboptimal. Initial Paramedic forms were included in 58\% of transferred charts. Primary Survey data (ABCDs) was present in 79\%-91\% of cases. C-spine status and Log Roll were documented in 59\% and $53 \%$ of charts, respectively. Documentation quality of ABCDs was variable. Overall adequate quality of Airway and Disability variables was $47 \%-83 \%$ while Breathing and Circulation was $86 \%-$ $91 \%$. Laboratory investigations were documented adequately in $63 \%$ of transfers. Only $34 \%$ of charts included transfer summaries from sending clinicians; $19 \%$ were of adequate quality. Up to 5 parts of the chart had to be assessed to obtain critical clinical information. Conclusion: The current process for transfer of major trauma patients in $\mathrm{BC}$ has sub-optimal communication, inefficiencies and is high risk for adverse events. A standardized trauma transfer protocol may improve patient safety.

Does the "acute care surgery" model allow care according to modern clinical practice guidelines to be achieved? An institutional practice evaluation. P. Murphy, ${ }^{*}$ D. Paskar, ${ }^{*}$ J. Racz, ${ }^{\dagger}$ N. Parry, ${ }^{\dagger}$ K. Leslie, ${ }^{\dagger}$ T. Mele. ${ }^{\dagger}$ From *Western University, and tLondon Health Sciences Centre, London, Ont.

Background: Current practice guidelines for the management of gallstone pancreatitis (GSP) recommend early cholecystectomy (EC) upon patient stabilization, preferably on the index admission. Previous work has shown that our institution has not been able to achieve this in most cases. A lack of emergency surgical resources was identified as a probable barrier. This study seeks to investigate whether the implementation of an "acute care surgery" (ACS) model results in higher quality care for GSP patients. Methods: For 2010 to 2013, patients admitted with GSP and eligible for EC were identified upon review of clinical, biochemical and radiographic criteria. Data was collected regarding demographics, admissions, timing of surgery and complications. A priori tests of statistical significance were performed to evaluate whether ACS implementation at 1 campus has resulted in improved care compared with non-ACS campus. Results: For 2010-13, 190 patients were identified as having GSP and candidates for EC. On the index admission, $66.7 \%$ received inpatient cholecystectomy at the ACS campus compared with $25.5 \%$ at the non-ACS campus. Of those not receiving index cholecystectomy, 16.0\% required a least 1 further admission for recurrent GSP. Overall, over $75 \%$ of patients went on to have a cholecystectomy in our institution - 60\% as inpatients. Conclusion: This study demonstrates that adoption of ACS achieves the standard of care for GSP compared with a non-ACS campus.

To drain or not to drain? Outcomes associated with pleural drainage of traumatic hemothoraces. B. Wells, ${ }^{*}$ D. Roberts, ${ }^{*}$ P. Navsaria, ${ }^{\dagger}$ S. Grondin, ${ }^{\neq}$A. Kirkpatrick, ${ }^{\ddagger}$ 
C. Ball. ${ }^{*}$ From the *University of Calgary, Calgary, Alta., tUniversity of Cape Town, Cape Town, South Africa, and fFoothills Medical Centre and University of Calgary, Calgary, Alta.

Background: South African data suggest that many traumatic hemothoraces (HTXs) can be managed expectantly. We sought to characterize the outcomes associated with pleural drainage of HTXs at a high volume Canadian level 1 trauma centre. Methods: A retrospective adult cohort study compared outcomes among major trauma patients (Injury Severity Score [ISS] $\geq 12$ ) with a HTX managed with or without pleural drainage (PD). Logistic and log-linear regression, adjusted for age, ISS, instability, and Glasgow Coma Scale, analyzed for differences in the odds of mortality and length of hospital stay (LOS) between groups. Results: Of 808 patients with a HTX between 2004 and 2012 (median age $45 \mathrm{yr}$; median ISS 25), 530 (66\%) underwent PD, while 278 (34\%) were managed expectantly (noPD). The PD group was more likely to have suffered a penetrating injury, as well as having required intubation and ventilation. Independent predictors of PD included number of ventilation days $(p=0.05)$, ipsilateral flail chest $(p=0.005)$ and/or pneumothorax $(p<0.001)$, and hemothorax size $(p<0.001)$. There was no adjusted difference between groups in the duration of mechanical ventilation $(1.15 \mathrm{~d}, 95 \%$ CI $0.73-1.82 \mathrm{~d}, p=0.54)$ or intensive care unit (ICU) stay ( $1.37 \mathrm{~d}, 95 \%$ CI $0.94-1.99 \mathrm{~d}, p=0.10)$. The PD group spent 1.58 days longer in hospital (95\% CI 1.37-1.81 d, $p<$ $0.001)$. The adjusted odds of mortality was not significantly different between groups (PD v. no PD, OR 0.19, 95\% CI 0.027$1.30, p=0.09$ ). Conclusion: Expectant management of HTXs was associated with a shorter hospital LOS with no increase in mortality, ventilator days, or ICU LOS. Further research will include a randomized controlled trial to evaluate the use of $\mathrm{PD}$.

Quality assurance within a point of care ultrasound curriculum: a pilot project. R. Hilsden, ${ }^{*}$ N. Parry, ${ }^{+}$ R. Arntfield. ${ }^{\dagger}$ From *Western University, and the TLondon Health Sciences Centre, London, Ont.

Background: Focused assessment with sonography for trauma (FAST) has now emerged as a standard imaging modality used during trauma resuscitation. Despite this, few general surgery residency programs have established formal curriculums for FAST ultrasound. Methods: A pilot FAST curriculum was developed with resident leadership and local subject matter experts at a level 1 trauma centre. The curriculum involved didactic teaching, handson training, and longitudinal quality assurance (QA) of recorded ultrasound imaging. Feedback on imaging generated during the care of patients was provided to the residents. Diagnostic accuracy was classified relative to expert interpretation, and image acquisition quality was classified as: no concerns (level 1), standards met with improvement suggestions (level 2), standards not met with concerns (level 3) and standards not met with serious concerns (level 4). Results: From January 2013 to September 2013 a total of 4 post-graduate year 1 residents participated in the pilot project. These residents submitted 34 studies for QA. 3\% of exams were level 1,72\% were level 2,25\% were level 3 and none were level 4 studies. Seventeen studies were classified as true negatives, 4 studies were false positives, 5 studies were true positives and no studies were identified as false negatives. Eight studies were indeterminate.
This resulted in a relative sensitivity and specificity of $100 \%$ and $81 \%$ respectively. Conclusion: This pilot project demonstrates the feasibility and value of a novel QA program as part of a FAST curriculum for general surgery residents.

Determinants of significant functional impact of minor thoracic injuries. M. Emond, J.-M. Chauny, M.-J. Sirois, E. Bergeron. From the Université Laval, Laval, Que.

Background: Approximately $75 \%$ of patients with a minor thoracic injury are discharged after an emergency department visit, while significant functional limitations can occur in the weeks that follow. The objective was to investigate whether minor thoracic injury and determinants relate to subsequent functional limitations. Methods: The study design involved a 19-month prospective cohort study with a 90-day follow-up. The setting was four university-affiliated emergency departments. A total of 482 patients, 16 years and older, with an minor thoracic injury with or without rib fracture, who presented between Mar. 1, 2008, and Oct. 1, 2009, were recruited and assessed at initial emergency department visit, 14, 30 and 90 days post-injury. Main outcome measures: functional outcome was assessed using the SF-12 scale at 30 and 90 days post-injury. Results: From the cohort, $127(26.3 \%)$ were aged 65 or more. Overall, 147 patients (30.5\%) presented with at least one rib fracture and 59 subjects $(12.2 \%)$ with delayed hemothorax. At 90 days, $22.8 \%$ of patients still had severe or moderate disabilities on global physical health score. Patients with solely delayed hemothorax and no rib fracture had the lowest global physical health score ( 46.4 v. $61.1, p<0.01$, effect size $=-2.56$ ) compared with patients with simple minor thoracic injury. Their physical functioning score were also lower up to 90 days post-injury $(72.1 \mathrm{v}$. $85.3, p<0.01$, effect size $=-1.21$ ). Generally, functional limitations also increase with increments of number of rib fracture detected on radiograph. Outcomes were not different among patients aged 65 years or more when compared with their younger counterparts. Conclusion: In this large prospective study, severe to moderate disabilities were present in one patient out of 5 . The presence of delayed hemothorax and the number of rib fracture were associated with increased functional limitations after a minor thoracic injury. Physicians should address detection of delayed hemothorax and prevention of disability in their discharge instructions.

Trauma transfer networks in Ontario: identifying optimal, stable, and efficient inter-facility transfer strategies. D. Gomez, ${ }^{*}$ B. Haas, ${ }^{+}$K. Larsen, ${ }^{*}$ A. Alali, ${ }^{*}$ T. Iwashyna, ${ }^{*}$ R. McDonald, ${ }^{*}$ G. Rubenfeld,,$^{\mathcal{S}}$ A. Nathens. ${ }^{\mathcal{E}}$ From the *University of Toronto, Toronto, Ont., tSt. Michael's Hospital, Toronto, Ont., ¥University of Michigan, Ann Arbor, Mich., and §Sunnybrook Health Sciences Centre, Toronto, Ont.

Background: Almost two-thirds of severely injured patients in Ontario are transported directly from the scene of injury to a nontrauma centre (NTC), with many requiring subsequent transfer to trauma centre (TC) care. Given the absence of system-wide transfer guidelines or arrangements, we believe there might be opportunities for improving the trauma transfer network. Methods: Populationbased mixed-method analysis of inter-facility transfer practices for severely injured patients in Ontario. Geographic Information Systems network analysis was used to identify the optimal transfer strategy (destination: 1 of 9 TCs and transportation mode: ground $v$. 
rotary-wing v. fixed-wing) for each NTC. Existing transfer strategies were derived from the Ontario Trauma Registry (2005-2009). We identified adult ( $\geq 18 \mathrm{yr}$ ), severely injured (Injury Severity Score $\geq 12$ ) patients who underwent transfer from a NTC to a TC, destination and mode of transport were recorded. Transfer strategies for each NTC were categorized based on optimality (minimizing transfer times). Results: We identified 7201 severely injured adult patients who underwent transfer from 146 NTCs to 9 TCs during the study period, approximately 1500 trauma transfers per year. The number of patients transferred by each NTC ranged from 5 to 193 (median 44, IQR 17-76). NTCs transferred patients using a median of 4 (IQR 2-6) different transfer strategies, ranging from as low as 1 to as high as 12 transfer strategies per NTC. Less than two-thirds of NTCs were characterized as using an optimal transfer strategy (best destination and mode of transport). Conclusion: Opportunities exist to improve the efficiency of Ontario's trauma transfer network.

Predictors of delayed hemothorax following minor thoracic injuries. M. Emond, J.-M. Chauny, E. Bergeron, N. Le Sage, L. Moore. From Université Laval, Laval, Que.

Background: Patients admitted to emergency department (ED) for minor thoracic injuries (MTI) are possibly at risk of delayed hemothorax (DHx). Little is known about the epidemiology and characteristics of patient with MTI treated on an out-patient basis that have DHx. We aimed to evaluate the incidence of DHx postED and the risk factors associated. Methods: A multicentre prospective cohort study was conducted in 4 Canadian EDs from March 2008 to August 2010. All consecutive patients, 16 years and older, with MTI and discharged from ED were screen and eligible. All initial chest X-rays were reported normal. A standardized clinical and radiological evaluation was performed upon initial ED visit and repeated at 1 and 2 weeks. Phone interviews were done at 30 and 90 days. Outcome: the presence of delayed radiological diagnosis of hemothorax. Univariate and multivariate analyses were realized to obtain outcome measures. Results: Of the 989 recruited participants, $254(25.7 \%)$ were 65 years or older, 610 (6.1.6\%) were male and $304(30.9 \%)$, at least 1 rib fracture confirmed on radiological report. A simple ground-level fall was the most frequent mechanism of injury $(n=325$ [32.8\%]). Overall, 118 (11.9\%) had developed a DHx within the first 14 days of followup. Our multivariate model, after controlling for patient's age and comorbidities, found preradiologically confirmed rib fracture, motor vehicle accident and use of ASA to significantly increase risk of DHx. Odds ratios were 4.4 (95\% CI 2.6-7.1), 1.9 (95\% CI 1.1-3.2) and 1.6 (95\% CI 0.9-2.9), respectively. Conclusion: This first large prospective cohort study on nonhospitalized patients with MTI revealed a very high incidence of delayed hemothorax. Our results support the development of clinical decision rules to orient tailored follow-up for MTI patients in ED.

Quality indicators of trauma reception using new informative technology. M. Emond, ${ }^{*}$ J.-M. Chauny, ${ }^{\dagger}$ A. Lavoie, ${ }^{\dagger}$ N. Le Sage, ${ }^{*}$ L. Moore, ${ }^{*}$ C. Malo. ${ }^{\ddagger}$ From *Université Laval, Laval, Que., tInstitut national d'excellence en santé et services sociaux, Montréal, Que., and the ¥McGill University Health Centre, Montréal, Que.

Background: Major trauma patients admitted to the emergency department (ED) are sent to the resuscitation area. Quality indicators
(QI) are essential to evaluate delivery of care. New informative technology (IT) and clinical decision support system (CDSS) could help evaluate real-time intervention and prevent medical errors. Objective: to describe potential trauma QI during the resuscitation phase using new IT. Methods: A prospective cohort study was conducted at a Canadian level 1 trauma centre over a 1 -year period. All consecutive trauma patients triaged to resuscitation bay were included in the study. A new IT (ReaScribe+) has been guiding delivery of care while collecting real-time interventions and data. All vitals signs are automaticaly recorded for analyses. Univariate and multivariate analyses were realized to obtain outcome measures. Analyses were stratified according to the presence of hypotensive state on arrival (systolic blood pressure $<90 \mathrm{~mm} \mathrm{Hg}$ ). Median values were used to report delays of care delivery in minutes. Results: One hundred trauma patients were triaged to the resuscitation bay during the study period. Initial Glasgow Coma Scale scores were recorded at 3 minutes and FAST ultrasound were realized at 7 minutes from trauma reception. The first peripheral catheter was installed within 5 minutes, the first fluid bolus initiated at 10 minutes $(n=27)$. When intubation was required $(n=7)$, initiation of procedure was done in 8 minutes and realized at 14 minutes. Eight patients (8\%) were hypotensive on arrival, median delay of initiation of fluids bolus was 10 minute compared with 11 minute in nonhypotensive. Mean total fluid volumes were significantly different in hypotensive patients $(1750$ v. $875 \mathrm{~mL}$; $p<0.05)$. Transfusion was initiated at 18 minutes versus 24 minute nonhypotensive trauma patients. Mean total transfusion volumes were significantly different in the hypotensive group $(1750$ v. $230 \mathrm{~mL}, p<$ $0.05)$. Conclusion: Specific quality indicator of care in major trauma reception and resuscitation could be obtained using new IT device in the ED. Delivery of care seems to be different for important QI using CDSS at bedside. Further studies are needed to validate QI in major trauma patients using new real-time intervention monitoring.

Using mixed methods to assess the knowledge, practices and comfort levels of occupational therapists, physiotherapists and nurses in mobilizing patients with traumatic pelvic fractures. M. McCaffrey, ${ }^{*}$ L. Foster, ${ }^{*}$ P. Foster, ${ }^{*}$ A. Coates, ${ }^{*}$ N. McNamara, ${ }^{*}$ K. McGeagh, ${ }^{+}$C. La Selva, ${ }^{\dagger}$ C. Thomas, $^{\dagger}$ E. Reynolds, ${ }^{\dagger}$ B. Petrisor. ${ }^{*}$ From ${ }^{*}$ Hamilton Health Sciences Centre, Hamilton, Ont., and tMcMaster University, Hamilton, Ont.

Background: Clinical pathways for mobilization of patients with traumatic pelvic fractures (TPFs) in the acute care setting are lacking. The aims of this study were to describe current mobilization practices and assess the knowledge and comfort levels of occupational therapists, physiotherapists and nurses caring for patients with TPFs. Methods: We used a 2-part, mixed-methods design. An online survey was distributed to allied health professionals and nurses working in our level 1 trauma centre and hospitals in our catchment area. Focus groups were conducted to further explore barriers to mobilization. Results: A total of 145 respondents completed the survey. Few respondents (10\%) reported access to a clinical pathway for mobilizing patients with TPFs and most (70\%) have never been taught a systematic approach to mobilization. Pain control and unclear activity orders were identified as barriers to mobilization. Lack of comfort in handling skills to mobilize patients in and out of bed and use of specialized equipment were identified by nursing. Qualitative data confirmed these barriers. We conducted 6 focus groups. Participants identified the 
need for a multidisciplinary approach and identification of clinician roles as key components to developing a clinical pathway. Conclusion: Our findings confirm the lack of a standardized approach to mobilizing patients with TPFs. Study findings will inform educational initiatives to address knowledge gaps, and identify methods for the development of an acute care clinical pathway for mobilization.

Blunt liver injury - Should we still perform serial hemoglobin? N. Kulvatunyou, R. Friese, L. Gries, D.J. Green, B. Joseph, T. O'Keeffe, A. Tang, G. Vercruysse, P. Rhee. From the University of Arizona, Tucson, Ariz.

Background: Serial hemoglobin (SH) has been a part of blunt liver injury (LI) management, but it does not affect the outcome. A significant bleeding occurs very early and delayed bleeding is very unusual. We hypothesized that non-serial hemoglobin (NSH) would not adversely affect the outcome. Methods: We queried our trauma registry for patients who presented to our level 1 trauma centre with blunt LI during 2009 to 2012. We excluded patients who were transferred from outside facilities, patients who underwent any surgical interventions within 24 hours, and any patients who had nonabdominal organ abbreviated Injury Severity Score (AIS) over 3. We defined SH group as those who received frequent blood drawn every 4-6 hours in the first 24 hours, and we compared the outcomes to the NSH group. The primary outcome was a failure of nonoperative management, defined as a requirement of surgical intervention or embolization. The secondary outcomes were total number of blood drawn within 24 hours, and the drop in hemoglobin level within 24 hours $(\Delta)$. Results: During the study period after the exclusion, 59 patients were in the $\mathrm{SH}$ group; and 37 patients in the NSH group. Outcome comparison is shown in the Table. Conclusions: Despite limited by small sample size, the study has shown that NSH in patients with blunt LI did not adversely affect the management or outcome. Hemoglobin always drifted down during the first 24 hours and that did not mean patients were bleeding. A larger prospective study is needed for the future.

\begin{tabular}{|c|c|c|c|}
\hline \multirow[b]{2}{*}{ Factor } & \multicolumn{2}{|c|}{ Group; mean $\pm \mathrm{SD}$ or median $(\mathrm{IQR})$} & \multirow[b]{2}{*}{$p$ value } \\
\hline & $\mathrm{SH}, n=59$ & $\mathrm{NSH}, n=37$ & \\
\hline Age, yr & $30 \pm 17$ & $45 \pm 20$ & $<0.001$ \\
\hline Sex, male; \% & $53 \%$ & $62 \%$ & 0.4 \\
\hline Injury Severity Score & $20 \pm 9$ & $18 \pm 9$ & 0.3 \\
\hline Glasgow Coma Scale & $15(14-15)$ & $15(14-15)$ & 1.0 \\
\hline Liver injury grade & $2(2-3)$ & $2(1-2)$ & $<0.001$ \\
\hline Grade I-II, no. & 33 & 31 & \\
\hline Grade III, no. & 21 & 6 & \\
\hline Grade IV-V, no. & 5 & 0 & \\
\hline $\begin{array}{l}\text { Failure of nonoperative } \\
\text { management }\end{array}$ & 0 & 0 & N/A \\
\hline $\begin{array}{l}\text { No. of blood drawn per } \\
24 \mathrm{~h}\end{array}$ & $5 \pm 1$ & $2.5 \pm 0.6$ & $<0.001$ \\
\hline $\begin{array}{l}\text { Drop in hemoglobin } \\
\text { within } 24 \mathrm{~h}(\Delta), \mathrm{g} / \mathrm{dl}\end{array}$ & -2.4 & -1.4 & 0.005 \\
\hline \multicolumn{4}{|c|}{$\begin{array}{l}\text { IQR = interquartile range; } \mathrm{N} / \mathrm{A}=\text { not available; } \mathrm{NSH}=\text { nonserial hemoglobin; } \\
\mathrm{SD}=\text { standard deviation; } \mathrm{SH}=\text { serial hemoglobin. } \\
\text { *Unless otherwise indicated. }\end{array}$} \\
\hline
\end{tabular}

Geographic disparities in helmet use among cyclists in montréal: an iPhone-based observation of 17000 cyclists. D. Bracco, T. Grenier, D. Deckelbaum, D. Mulder, K. Khwaja, T. Razek. From McGill University Health Centre, Montréal, Que.

Background: The objective of the present study is to assess the regional disparities in helmet use among cyclists in Montréal. Methods: An iPhone based app was developed to perform a cross sectional survey from June 1 to October 15, 2013, and distributed to volunteer observers. Date, time and GPS position was gathered from the iPhone and LatLong was sent to Google API for reverse geomapping. Standard statistical methods were used. Results: Twenty-two observers reported on 17246 cyclists in 1987 different locations. The hemet wearing proportion was $47.8 \%(47.1 \%-48.6 \%)$. Women $(50.7 \%)$ wore helmets more often than men (43.9\%). Bike sharing program users $(14.8 \%)$ and visible minorities $(25.8 \%)$ had low compliance. Children $(63.5 \%)$ and seniors $(55.8 \%)$ wear helmets more often than adults (47.0\%). There was highly significant geographic east to west gradient (Figure) in helmet use, each kilometre westward is associated with a $1.66 \%$ increase in helmet use $\left(\chi^{2}=131\right)$. There was a correlation between socioeconomic status and helmet compliance, (Figure) with a 5\% decrease in helmet compliance for every $10 \%$ increase in the population below poverty line $\left(\chi^{2}=191\right)$. Conclusion: Helmet use by cyclists is still below $50 \%$ in Montréal. Specific targets for prevention should include young adults, visible minorities, males, and the bike sharing program as well as lower socioeconomic areas.

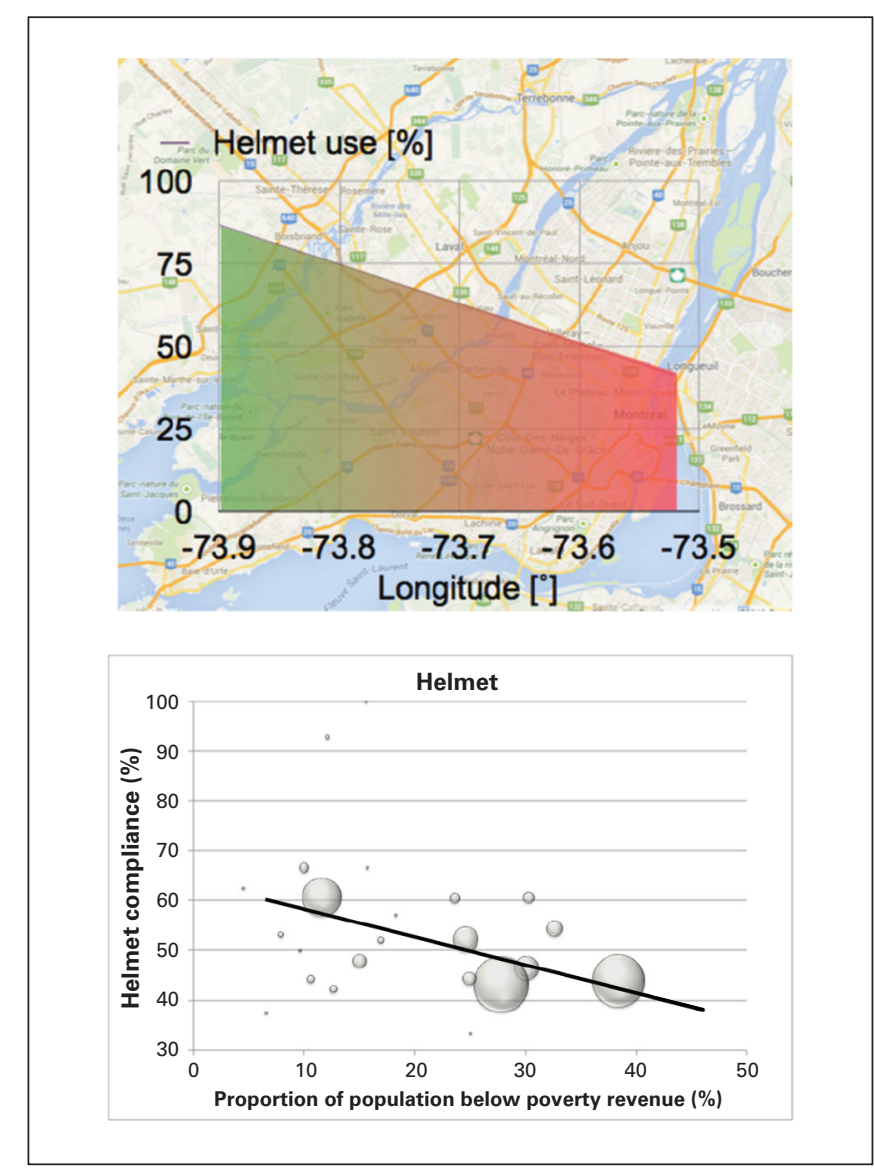


Deployment of an iPad-based core data trauma registry in Dar es Salaam, Tanzania. A. Lalande, ${ }^{*}$ T. Razek, D. Bracco, ${ }^{*}$ D. Deckelbaum, ${ }^{*}$ V. Munthali, ${ }^{+}$R. Boniface, ${ }^{+}$ L. Museru. ${ }^{\dagger}$ From *McGill University Health Centre, Montréal, Que., and the TMuhimbili Orthopedic Institute, Dar es Salaam, Tanzania

Background: Injuries are responsible for 1 in 10 deaths worldwide. Low- and middle-income countries are most severely affected by injuries, bearing $90 \%$ of the worldwide mortality burden. Increasing use of trauma registries has been instrumental in decreasing injury mortality, by allowing for better prevention and trauma system design. Methods: An iPad app, iTrauma, was developed in Montréal and implemented at the Muhimbili Orthopedic Institute (MOI) to improve the efficiency of data collection for an already existing injury database. Information acquired includes origin of the patient, nature and cause of the injury and outcome in hospital. The field-testing took place at MOI this summer. We assessed its efficiency by measuring the time to enter one patient file in iTrauma. Results: iTrauma was successfully developed and implemented in MOI, Dar es Salaam. The test database contains 447 records and records could be entered in 90 to 120 seconds per case. This kind of database automatically uploads to an excel file which produces regular almost real time dashboard reports. Conclusion: This efficient method of data acquisition is feasible in the low resource environment of Dar es Salaam and can be expanded across the globe. Core trauma data are a very powerful tool to understand and improve the burden of injuries in low-income areas. Future implementation of this simplified data set in various environments is underway.

Indication for CT scanning in the initial evaluation of trauma patients: image the injuries, not the weapon. D. Bracco, Y. Lin, M. Azzam, J. Al-Oweis, A. Roman, D. Deckelbaum, K. Khwaja, P. Fata, T. Razek. From the McGill University Health Centre, Montréal, Que.

Background: Computed tomography (CT) scanning has become an integral part of the evaluation of a trauma patient. The aim of the study is to prospectively assess the clinical indication and usefulness of CT in trauma patients. Methods: Over 1 year, the indications, clinical findings and clinical decision making based on the CT results were observed for CT of the head, cervical spine (C-spine), chest and abdomen/pelvis during initial trauma work-up. The a priori indication for CT was classified based on mechanism, physiologic or anatomic findings. Results: Over 1 year, 474 patients were evaluated: rates of CT head, Cspine, chest and abdomen/pelvis were $90 \%, 76 \%, 67 \%$ and $66 \%$, respectively. The rate of positive CT was variable across different body area and CT indications (Figure). Computed tomography C-spine and mechanism of injury had lower yields of positive findings. Mechanism of injury combined with nonexaminable patients had an odds ratio of $0.37(0.35-0.38, p<0.0001$, Number Needed to Treat 4 [3-6]) of a positive CT when compared with anatomic and physiologic indications to CT. A total of $5.5 \%$, $3.3 \%, 2.4 \%$ and $6.2 \%$ required surgical intervention based on CT after head, C-spine, chest and abdomen imaging, respectively. Rate of incidental findings were $3 \%, 2.6 \%, 12 \%$, and $22 \%$, respectively for head, C-spine, chest and abdomen CT scans. Conclusion: Anatomic and physiologic indication for scanning had the highest finding rate. Negative imaging could help discharge patients from the emergency department.

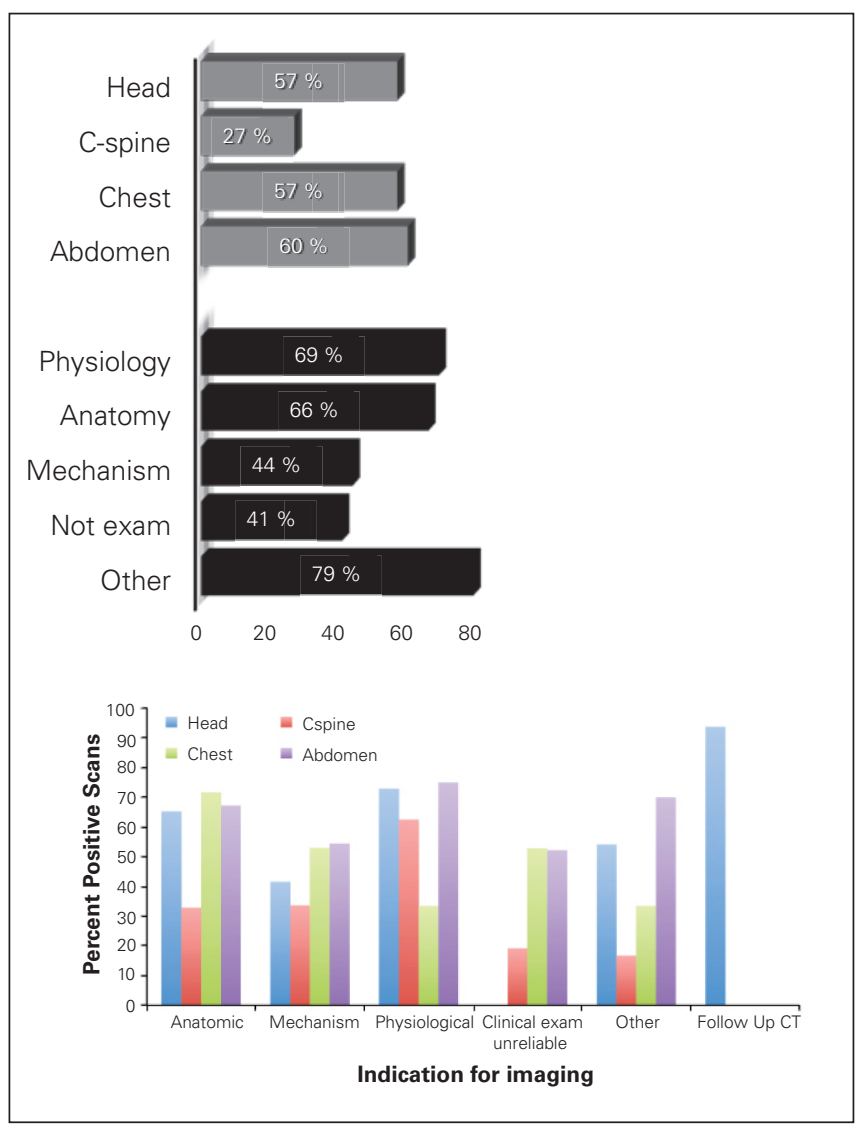

The road to trauma distinction: a partnership between accreditation Canada and the Trauma Association of Canada to design an accreditation program. T. Taulu, ${ }^{*}$ D. Sarakbi, ${ }^{\dagger}$ L. Phillips, ${ }^{\dagger}$ J. Tallon, ${ }^{*}$ P. Poirier, ${ }^{\ddagger}$ R. Simons, ${ }^{*}$ S. Rizoli, ${ }^{\mathcal{E}}$ F. Brenneman, ${ }^{\prime \prime}$ B. MacDonald. ${ }^{\dagger}$ From *Trauma Services, Vancouver General Hospital, Vancouver, BC, †Accreditation Canada, Ottawa, Ont., ‡EMS Nova Scotia, Dartmouth, NS, §St. Michael's Hospital, and the ๆSunnybrook Health Sciences Centre, Toronto, Ont.

Background: Since the inception of the Trauma Association of Canada (TAC) in 1983, it has been recognized that a structured evaluation program is required in order to measure, advance and improve trauma care in Canada. Since 1995, TAC has been surveying and accrediting trauma services in Canada. In 2008, TAC and Accreditation Canada began discussions about a partnership for accrediting trauma systems in Canada and in 2012, TAC and Accreditation Canada signed a formal Memoranum of Understanding and established a steering committee to oversee the transition and development of a national accreditation program housed within Accreditation Canada and using the expertise of trauma experts through TAC. Methods: In 2012, Accreditation Canada and TAC developed a critical path and detailed work plan including a comprehensive communication plan, regular trauma steering and advisory committee meetings, and regular meetings of small working groups. The Trauma Distinction program consists of requirements for meeting standards of excellence and performance 
indicator thresholds, having protocols in place, and having an excellence and innovation project or initiative. The program was evaluated through national consultation and pilot testing at the site and system level. Results: Following the 18 month work plan, Accreditation Canada has successfully led the development and evaluation of the Trauma Distinction program in partnership with TAC, and will be seeking board approval and full nation-wide program release in the fall of 2014. Conclusion: The transition of trauma accreditation was an opportunity for TAC and Accreditation Canada to capitalize on their respective strengths as experts in trauma services, and in development and ongoing maintenance of accreditation programs. This has ensured that best practices and experience are the foundation of the new Trauma Distinction program.

A multifaceted quality improvement strategy to optimize monitoring and management of delirium at a level I trauma centre: phase I results. S. Faidi, ${ }^{*}$ P. Foster, ${ }^{*}$ A. Coates, ${ }^{*}$ N. McNamara, ${ }^{+}$M. Bhandari. ${ }^{+}$From ${ }^{*}$ Hamilton Health Sciences Centre, and +McMaster University, Hamilton, Ont.

Background: Delirium is a common clinical complication in trauma patients. Knowledge of risk factors allows for early detection and implementation of prevention and management strategies. A survey conducted of nurses and allied health professionals at our level 1 trauma centre found considerable variability in the knowledge of delirium. Respondents agreed that regular assessments are important to detect delirium; however, few routinely administered the confusion assessment method (CAM) on their patients. Staff also reported a lack of confidence in managing patients with delirium. Survey results informed the selection of quality improvement (QI) interventions to improve monitoring and management of delirium in trauma patients. Methods: Our interventions included educational workshops, audit and feedback, and semistructured interviews to identify barriers to routine monitoring. Hospital charts were reviewed to determine the incidence of delirium and assess CAM compliance rates. Results: Workshops were well attended with participation rates of $74 \%$ and $78 \%$. Audit and feedback results from phase I to phase II showed improvement in CAM compliance from $16 \%$ to $68 \%(p<0.05)$. Tailoring interviews confirmed staff are aware of CAM, agree that routine monitoring is important, and have adopted the new practice, albeit irregularly. Staff consistently identified computer issues and lack of time as barriers to adherence. Conclusion: Staff were engaged in this QI project. Initiation of our delirium quality improvement strategy was associated with marked improvements in compliance with CAM.

Tube the patient until he is sober: impact of alcohol and drug use among trauma patients on outcome and resource use. D. Bracco, D. Roizblatt, C. Malo, D. Deckelbaum, T. Razek. From McGill University Health Centre, Montréal, Que.

Background: Alcohol and drug use are known trauma risk factors. The aim of this study is to evaluate the effect of alcohol and drug consumption on trauma outcome and resources utilization. Methods: The trauma registry was cross-referenced with the laboratory information system, and any patient flagged as alcohol or drug in the trauma database or with a positive alcohol/drug plasma level upon admission was considered positive. Models predicting need for ventilation, including Injury Severity Score (ISS), age and alcohol, were tested. Results: A total of 1220 out of 7413 (16.5\%) patients over 5 years were positive. The ethanol positive patients were more likely to be male (79.4\% v. $59.7 \%$ ), younger (median 39 v. $56 \mathrm{yr})$, have penetrating injuries (16.5\% v. 7.1\%). Corrected for ISS and age, drunk patients had an OR of 2.31 (95\% CI 1.98-2.70), requiring ventilation. Across all ISS strata alcohol increased the risk of requiring ventilation (Figure). The low ISS group had an OR of 3.95 (95\% CI 3.70-4.18), requiring ventilation, 3.01 (95\% CI $2.83-$ 3.20 ) in the ISS 10-15 group, 2.64 (95\% CI 2.53-2.75) for ISS 1625 and 1.98 (95\% CI 1.93-2.03) in the ISS above 25 group. Among ventilated patients, positive patients had the same lung open ventilation (LOV) as sober patients. Alcohol increased the intensive care unit admission rate (OR 1.32, 95\% CI 1.13-1.56; $p=0.0006)$. Alcohol doubled the ISS-corrected mortality (OR 2.03, 95\% CI 1.54 2.74). Conclusion: Intoxicated patients require significantly more ventilatory support than sober counterparts, but do not require short ventilation time "until sober." Patients intoxicated with alcohol or drug use a significant amount of resources and associated with increased mortality.

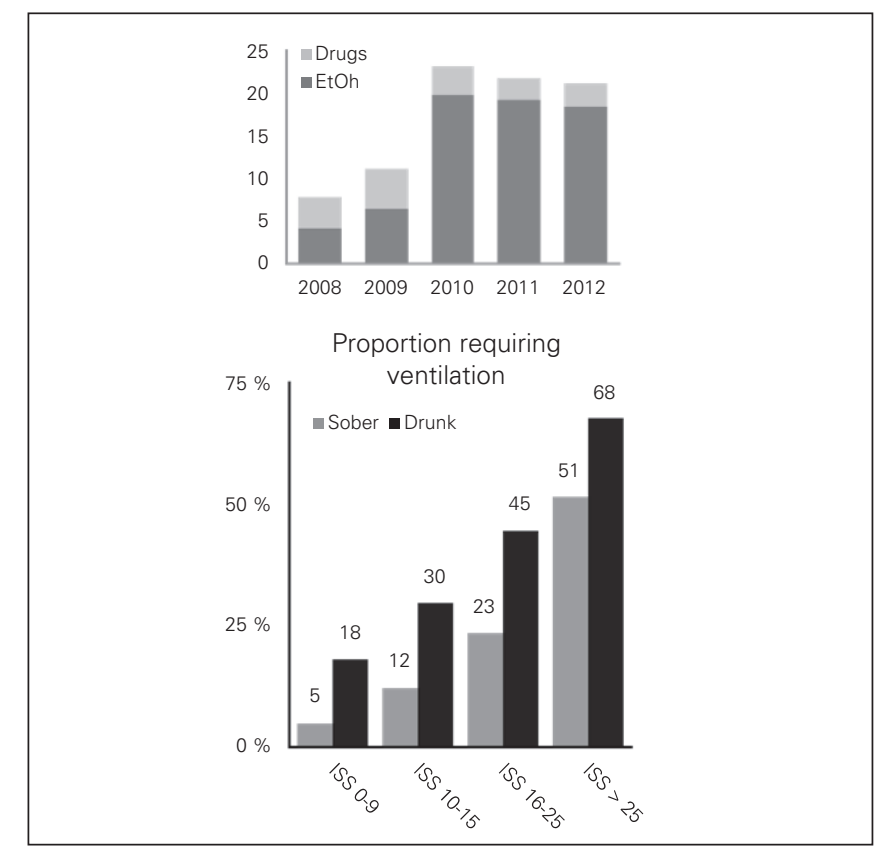

Variability in the management of blunt splenic injuries according to facility level: a state-wide study from the Quebec Trauma Registry. T. Bege, R. Denis. From Montréal Sacré Coeur Hospital, Montréal, Que.

Background: Nonoperative management can be achieved in the majority of splenic blunt injuries. However, previous studies have noticed a high variability use of surgery. This study evaluated the influence of facility level and interfacility transfer on splenic trauma management. Methods: A retrospective study was performed from the Quebec Trauma Registry between 2006 and 2011, including all adult patients (aged 16 and over) with a blunt splenic injury. Multivariate models involving age, Injury Severity Score, and grade were used to analyze mortality, use of surgery and embolization. Results: There were 1596 patients identified in 70 different facilities. The management was initiated in nearby hospital (Quebec level 1 or 2) in $67 \%(n=1074)$ and in specialized trauma centre (Quebec regional 
level 2 or 3$)$ in $33 \%(n=522)$ of cases. Among patients initially managed in nearby hospital, $31 \%(n=333)$ were transferred to a specialized trauma centre. Factors independently associated with a transfer were ISS and splenic injury grade. There was no significant difference in adjusted mortality according to facility level. However, patients transferred and directly admitted in a specialized centre had a lower adjusted rate of surgery (respectively, OR $0.3[0.2-0.5]$ and OR $0.3[0.4-0.8]$ ) and a higher rate of embolization (respectively OR 2.8 [1-7.8] and OR 4 [1.7-9.8]) than patients who remained in a nearby hospital. Conclusion: Differences in management of blunt spleen trauma have been highlighted according to level of facility. Direct admission or transfer to a specialized trauma centre is associated with a lower rate of surgery and higher rate of embolization.

Systematic evaluation of trauma transfers to a level 1 hospital: the chain's weak link. D. Bracco, Y. Lin, M. Azzam, C. Malo, A. Roman, D. Deckelbaum, T. Razek. From McGill University Health Centre, Montréal, Que.

Background: Interfacilities transport is a crucial component of a regionalized trauma system's ability to provide definitive care in a timely fashion. The objective of the present study is to prospectively evaluate the logistic and clinical aspects of trauma transport in a regionalized trauma system. Methods: Dispatching information was logged in real time on a dedicated smartphone application by the trauma team leader on-call. Upon arrival, the vital functions, the logistic aspects and the required interventions were recorded for transfers from the island of Montréal, Southern and Northern Quebec to the Montréal General Hospital. Safety and speed of transport were assessed. Results: A total 336 trauma transfers were prospectively evaluated. Mean transport initiation and mean total transport times were much longer than expected, especially for patients transferred from Northern Quebec compared with Southern Quebec and the island of Montréal. Overall, 5.6\%, 3.1\% and 10\% of transferred patients needed airway, chest tube, and fluid/blood resuscitation immediately on arrival respectively. A minority of patients had large peripheral ( $\geq 16 \mathrm{G}$ ) or central venous access (12\%). Thirteen percent of transfers were considered unsafe. Conclusion: Trauma transfers are a weak link in our regionalized trauma system. A dedicated regional trauma transport system targeting transport times and safety should be implemented.

Frequency and severity of concussion symptoms following major trauma. B. Moffat, ${ }^{*}$ T. Charyk Stewart, ${ }^{+}$ K. Vogt, ${ }^{\ddagger}$ E. Barrett, ${ }^{\dagger}$ M. Khami, ${ }^{\dagger}$ N. Parry. ${ }^{\dagger}$ From the *Western University, tLondon Health Sciences Centre, London, Ont., and ¥Los Angeles County and University of Southern California, Los Angeles, Calif.

Background: Concussion symptoms can be long-lasting and functionally debilitating. The symptoms can be difficult to detect and diagnose while in hospital. This study was undertaken to determine the frequency and severity of concussion symptoms among major trauma patients following discharge from hospital. Methods: Patients followed in trauma clinic from February 2012 through March 2013 at 2-4 weeks post-discharge were screened using the Rivermead post-concussion symptom questionnaire (RPQ). Composite scores reflect severity of early and late post-concussion symptoms. The RPQ scores were combined with data from our prospectively collected trauma database. Patients were divided into those with traumatic brain injury (TBI) diagnosed on CT and those with a normal CT head on admission. Results: A total of 90 charts were analyzed. Consistent with most trauma studies, males were more likely to incur TBI than females. Most of the head injuries were caused by falls, $65.5 \%$ of the admissions were TTA and $59 \%$ arrived directly from the scene. The average time to CT from admission was 4 hours 36 minutes, with $21.1 \%$ obtaining a CT scan within 1 hour. A total of $28.9 \%$ of the patients expired in hospital. Trauma activations had $65.4 \%$ mortality. Conclusion: Nontrauma activation admissions experienced longer wait times for CT and were mainly referrals. A total of $65.8 \%$ arrived with CT completed within 4.5 hours of admission from the referring facility. While the facility has one of the lowest mortality rate among North American trauma centres, the deaths associated with transfers is fairly high. More timely diagnosis in TBI and appropriate intervention may reduce the mortality of this group.

Consequences of delayed trauma team activation. B. Moffat, ${ }^{*}$ D. Mainprize, ${ }^{\dagger}$ K. Vogt, ${ }^{\ddagger}$ T. Charyk Stewart, ${ }^{\dagger}$ N. Parry, ${ }^{\dagger}$ D. Gray. ${ }^{\dagger}$ From *Western University, London, Ont., tLondon Health Sciences Centre, London, Ont., and fLos Angeles County and University of Southern California, Los Angeles, Calif.

Background: The availability and mobilization of a structured trauma team for critically injured patients has long been a mainstay in advanced trauma care systems. Despite clear guidelines for activation at most trauma centres, delays in trauma team activation (TTA) still occur. We sought to identify the impact of such delays on patient survival and outcomes as well as the predictors of delayed TTA. Methods: Consecutive patients admitted to our Canadian level 1 trauma centre from April 2007 to February 2013 were identified using our prospectively collected trauma database. TTAs occurring during this time were matched to the corresponding patients. Regression analysis was performed to identify factors associated with TTA delay. Results: There were 1000 TTAs over the study period. A total of $158(16 \%)$ and 68 (7\%) were delayed by 10 minutes and 30 minutes or more, respectively. Lower ISS was associated with TTA delay (18 v. $22, p<0.01)$. Patients arriving direct from scene were more likely to have TTA delay ( $72 \%$ v. $44 \%, p<0.01)$. Penetrating injuries were more likely to receive advanced notice (OR 3.55, 95\% CI 1.93-6.53). TTA delay or advanced notice were not predictive of mortality or complications. ISS, age, and initial vitals were not associated with TTA delay on regression analysis. Conclusion: The TTA delay does not seem to be associated with increased mortality. Likewise, advanced notice before patient arrival does not seem to improve survival. More stable patients are more likely to have TTA delay which may account for the decreased mortality in this group.

Age of blood has no impact on trauma outcome. D. Bracco, A. Wilson, D. Deckelbaum, T. Razek. From McGill University Health Centre, Montréal, Que.

Background: Data on the age of transfused blood suggest worse outcome associated with older blood in cardiac surgery, but data outside this field has yielded mixed rests. The objective of the present study is to observe the age of blood used in our centre and the effect 
of the age of blood on outcome in trauma patients. Methods: The trauma database was cross-referenced with the blood bank database and data over 5 years (2008-2013) were reviewed. Results: Over 5 years, 1633 (22\%) out of 7413 trauma received a total of 9637 packed red blood cells. Most of the blood was over 13 days and the median age of blood unit received by trauma patients was 22.5 days and the median oldest blood received by each patient was 29.7 days. In patients receiving numerous units of blood, the median age had a tendency to decrease reflecting lower stocks in the blood bank. A minority $(n=83)$ received exclusively young blood. After adjustment for Injury Severity Score and age, the age of blood had no effect on mortality. When comparing the old only versus young only group, young blood receiving patients had a similar outcome (OR 0.920, 95\% CI 0.44-1.75 normal saline). Conclusion: Predominantly old blood units are used in our trauma patients. The age of the blood had no effect on trauma outcome in the present setting.

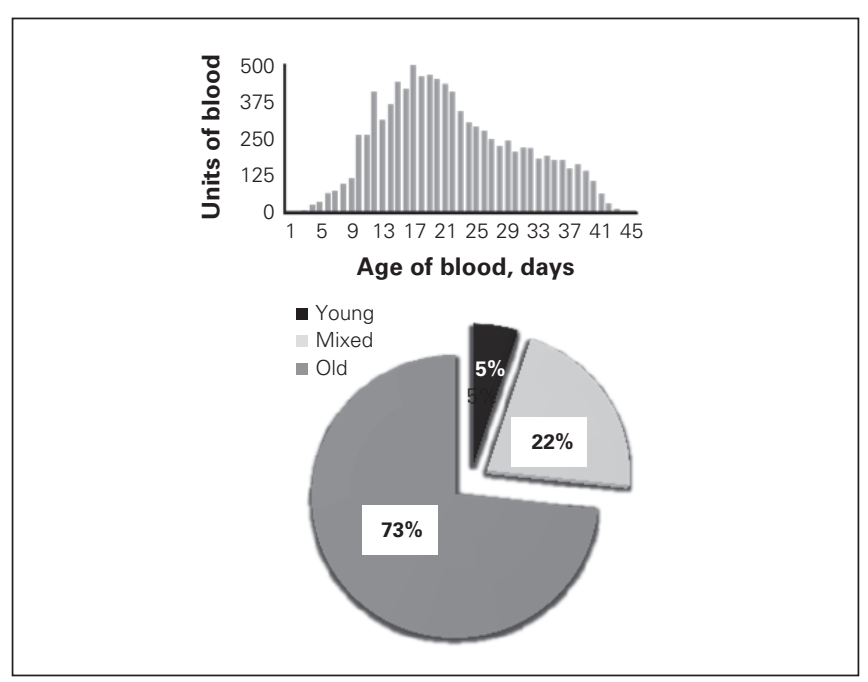

\begin{tabular}{|lcc|}
\hline Table. & Estimate & $p$ value \\
\hline Outcome (Alive) & $0.952(0.942-0.962)$ & $<0.0001$ \\
\hline Injury Severity Score per point & $0.980(0.973-0.986)$ & $<0.0001$ \\
\hline Age per yr & $1.016(0.998-1.035)$ & 0.08 \\
\hline Age of blood per d & \\
\hline
\end{tabular}

Anticoagulation reversal: from not enough fresh frozen plasma to underdosed prothrombin complex concentrate. D. Bracco, D. Roizblatt, C. Malo, K. Khwaja, P. Fata, D. Deckelbaum, T. Razek. From the McGill University Health Centre, Montréal, Que.

Background: Fresh frozen plasma (FFP) and prothrombin complex concentrate (PCC) have been used to reverse anticoagulation, with the latter being preferred for its faster action and lower volumes administered. Is Octaplex related to better survival rates and less admissions to the intensive care unit (ICU)? Methods: Data from our trauma bank registry was analyzed from 2008 to 2012. The inclusion criteria were all patients admitted to trauma who received FFP and/or PCC for anticoagulation reversal. Results: Over the 5-year period, 166 patients received FFP and/or PCC for anticoagulation reversal. Forty- two $(25.3 \%)$ received FFP, 113 (68.07\%) PCC and $11(6.63 \%)$ received both. We could see a trend of giving PCC over FFP since 2010 , with $85 \%$ of patients in 2012 receiving only PCC. Only $25 \%$ had a post-correction international normalized ratio (INR) below 1.3. The initial INR was higher $(p<0.005)$ between groups and was comparable after correction. Injury Severity Score-adjusted mortality was similar among the 3 groups. Thirty-three (78.57\%) patients receiving FFP, $66(58.41 \%)$ receiving $\mathrm{PCC}$ and $7(63.64 \%)$ receiving both where admitted to ICU $(p=0.06)$ with similar length of stay and ventilation. Conclusion: Admission to ICU, length of stay in ICU and survival are not dependent on what drug we use for anticoagulation reversal in trauma patients. Prothrombin complex concentrate seems to have better response comparing the initial INR with the 6 hours control, but it seems we are not reaching optimal INR due to under dosing it.

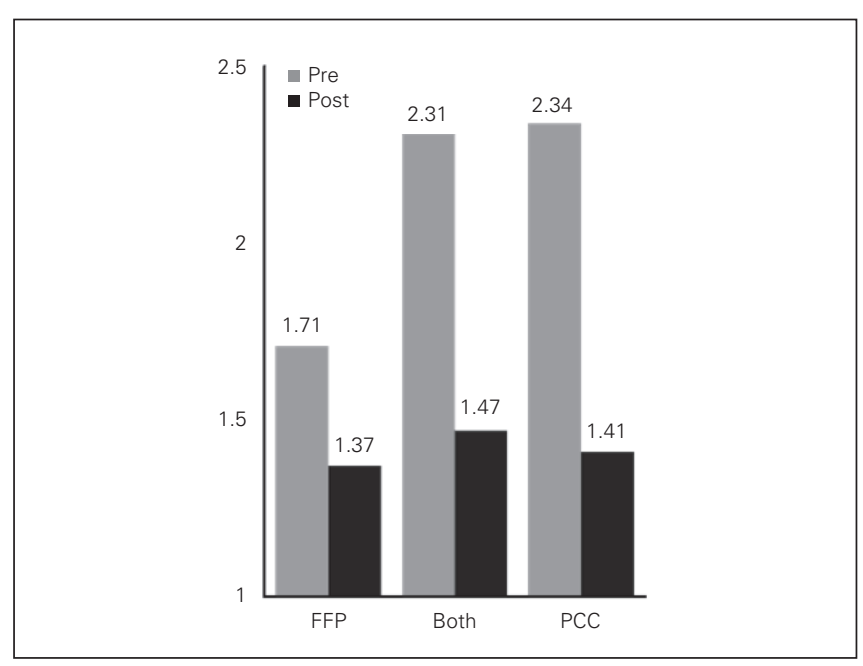

A retrospective review of time-to-analgesia for the direct from scene trauma patients transported to a level 1 trauma centre by a single EMS provider. A. Slomer, M. McGowan, K. Nixon, D. MacKinnon. From St. Michael's Hospital, Toronto, Ont.

Background: Providing timely and adequate analgesia is a challenging aspect of the care of patients with acute traumatic injuries. The objective was to describe time from first contact with a care provider and administration of analgesia in direct from scene trauma patients. Methods: A 6-month retrospective chart review (January-June 2012) of trauma team activations (TTAs) who were normotensive with Glasgow Coma Scale over 12 and transported direct from scene by a single municipal emergency medical services (EMS) provider to a level 1 trauma centre was conducted. Exclusions were intubation (prehospital or upon arrival), vital signs absent and without an ambulance call record in the chart. Clinical, demographic and administrative data were extracted and $10 \%$ independently reviewed for interrater reliability. Results: A total of 187 TTAs were identified; 70 direct from scene and 51 met inclusion. Five received pain relief prehospital (2 analgesia; 3 splinted for blunt-extremities). Thirty-seven (73\%) received analgesia upon arrival to the trauma room, with similar time to receiving analgesia irrespective of type or area of injury: penetrating-extremities (4 [80\%], $14 \pm 8 \mathrm{~min}$ ); penetrating-other 
(11 [58\%], $14 \pm 12 \mathrm{~min})$; blunt-extremities (10 [91\%], $15 \pm 9 \mathrm{~min})$; blunt-other (12 [75\%], $12 \pm 8 \mathrm{~min}$ ). Time to analgesia from first contact with care provider for the 35 patients who did not receive any prehospital relief was $40 \pm 14$ minutes. Conclusion: Despite a prehospital analgesia protocol for blunt extremity injuries, this review highlighted that most TTA patients fail to receive any pain relief in the prehospital setting. While the majority of TTAs do not fit existing EMS protocol, most were felt to be appropriate for and received timely analgesia upon arrival to the trauma room. Opportunities may exist to improve the trauma patient experience by providing more timely pain relief in the prehospital setting.

Albumin resuscitation in trauma patients: Does it increase mortality? C. Malo, D. Bracco, D. Roizblatt, J. Alowais, D. Deckelbaum, P. Fata, K. Khwaja, T. Razek. From McGill University Health Centre, Montréal, Que.

Background: The choice of resuscitation fluid for trauma patients has been an important debate in the literature. Albumin has been seriously challenged since studies have shown no decrease in mortality with its use and because of its higher cost. Methods: Data from our trauma and blood bank registries were cross referenced to identify albumin $5 \%$ or $25 \%$ administered to trauma patients. Settings, timing and patients descriptors of trauma receiving albumin were determined using the trauma registry. Comorbidities were determined using the Charlson Comorbidity score. Results: Over 5 years (2008-2013), 125 out of $7413(1.7 \%)$ trauma patients and $4.9 \%$ of intensive care unit (ICU)-admitted patients received albumin. Most of the albumin was used in the ICU. Albumin use was associated with higher Injury Severity Score (ISS) (median 21 v. 11) and age (65 v. 53 yr). Median serum albumin level before albumin administration was $18 \mathrm{~g} / \mathrm{L}$ (normal values: $35-52 \mathrm{~g} / \mathrm{L}$ ). Unadjusted mortality was $24.0 \%$ in albumin receiving patients (v. 6.6\%). Albumin was also used in low ISS patients. Albumin tripled ISS/age-corrected mortality with an odds ratio (OR) of 2.88 (95\% CI 1.80-4.50, $p<0.0001$ ). When adding Charlson Comorbidity score, albumin use increased mortality with an OR of 2.59 (95\% CI 1.62-4.06). In a model including ISS and age, albumin administration was associated with a 15 days longer hospital stay. Conclusion: Albumin is still used as fluid resuscitation in trauma patients almost exclusively in the ICU. Albumin use may be harmful in trauma patients.

Distal radial fractures: adequacy of reductions performed in the emergency department. R. Montazeri, ${ }^{*} \mathrm{~J}$. Wagg, ${ }^{+}$ J. Mayich, ${ }^{+}$H. King, ${ }^{+}$J. Swan, ${ }^{+}$J. Fraser, ${ }^{+}$S. Benjamin, ${ }^{,}$ P. Atkinson. ${ }^{\dagger}$ From *UNB - Dalhousie University, +UNB Dalhousie University, Saint John Regional Hospital, ‡Saint John Regional Hospital, and the §New Brunswick Trauma Program, Saint John, NB

Background: Distal radial fractures remain the most commonly encountered fracture in the emergency department (ED). Their initial management by emergency physicians (EP) poses considerable resource allocation. Methods: This study was a retrospective study on 70 patients who received procedural sedation and manipulation for a distal radial fracture. Radiological images performed at initial assessment, post-reduction, and on clinic follow-up of included patients were reviewed by a panel of orthopedic surgeons and radiologists and assessed for evidence of displacement. Results: Distal radius fractures were deemed adequately reduced in $53 \%$ of cases (95\% CI 41.32-64.10) immediately post-reduction. On follow-up assessment in Orthopedic clinic, 70\% of the fractures previously in a reduced state were still found to be adequately reduced, giving a $30 \%(95 \%$ CI 17.37-45.90) slippage rate in our patient population. Further intervention with operating room visit was eventually required in $10 \%$ (95\% CI 4.65-19.51) of our patients with distal radius fractures. Conclusion: We found an adequacy rate of $53 \%$, with displacement in $30 \%$ of cases on follow-up. A successful initial reduction was less likely to result in requiring further intervention. On assessment of radiographs for reduction adequacy, our panel of orthopedic surgeons and radiologists were in agreement $38.6 \%$ of the cases. We found a higher rate of females and patients aged greater than 65 years in our sample, with no statistical correlations found between age, sex, or of time of initial presentation to the ED and final outcomes.

Evaluation of tranexamic acid and thromboembolic events in trauma patients. B. Macanovic, H. Tien, M.C. Arci Santos, B. Nascimento. From the Sunnybrook Health Sciences Centre, Toronto, Ont.

Background: In 2010, the CRASH-2 study demonstrated the survival benefit of administering tranexamic acid (TXA) to trauma patients at risk of significant hemorrhage. However, due to the shortfalls of CRASH-2 in detecting thromboembolic events (TEE); and some military data suggesting increased risks of TEE, the safety of TXA remains undetermined. Our study evaluates the rate of TEE associated with TXA use in civilian trauma. Methods: Retrospective review was conducted from January 2011 to June 2013. All traumas who received at least 1 unit of packed red blood cells within the initial 24 hours were included. A TEE was defined as any episode of deep venous thrombosis (DVT), pulmonary embolism, myocardium infarction or stroke at any time during hospital stay. The TXA and nonTXA groups were analyzed for TEE rates. Results: The study identified 400 patients: 103 in the TXA and 297 in the non-TXA group. The groups were similar with respect to demographics, severity of injury and degrees of shock and coagulopathy. The rate of TEE in the TXA group was $13.6 \%$ and the non-TXA $8 \%$ $(p=0.143)$. The DVT rates were $6.7 \%$ in the TXA versus $3 \%$ in the non-TXA group $(p=0.162)$. Conclusion: Although not statistically significant, preliminary data suggests that the use of TXA in trauma is associated with increased thromboembolic complications. Overall, our study found higher rates of TEE than the CRASH-2. Due to the clinical significance of our findings, the safety of administrating TXA in trauma requires further investigation. The true causality remains to be determined.

Improving effectiveness of field trauma triage activations in New Brunswick. R.D. Boulay, ${ }^{*}$ D. Richard, ${ }^{*}$ J.-P. Savoie, ${ }^{*}$ E. Goulette, ${ }^{*}$ A. Chisholm ${ }^{\dagger}$ I. Watson. ${ }^{\dagger}$ From ${ }^{*}$ Ambulance New Brunswick, Moncton, NB, and the †New Brunswick Trauma Program, Horizon Health Network, Saint John, NB

Background: Field trauma triage (FTT) was implemented across New Brunswick in November 2010. The triage allows for 
bypass of level V designated facilities in favour of level I, II or III trauma centres for patients with qualifying injuries. While review of FTT application quality occurs regularly, interest has remained in determining if paramedics are activating the system for all qualifying trauma patients. This retrospective study aimed to determine changes in the relative rate of FTT activation. Methods: Data supplied by Ambulance New Brunswick for the number of FTT activations per year were compared with data from the Discharge Abstract Database for New Brunswick to determine relative rates of FTT activation. During the study period, system-wide education of all 1000 paramedics was undertaken, as well as focused follow-up on cases where patients were admitted with no record of FTT activation. Results: Provincially, 2012 to 2013 showed a significant increase of $7 \%$ in activations/hospital injury related discharges (1565 of 6137 [26\%]) versus the previous year (1168 of 6252 [19\%]; $\chi_{1}^{2}=83.361,2$-tailed $\left.p<0.0001\right)$. This provincial rate was similar to the increases in Saint John (8\%) and Moncton (9\%). Conclusion: Although not intended to provide an absolute measurement of FTT activations, calculation and monitoring of relative changes in FTT activation rates is helpful in demonstrating the impact of ongoing paramedic education. The NB Trauma Program continues to support Ambulance New Brunswick to ensure FTT activation for all qualifying trauma patients.

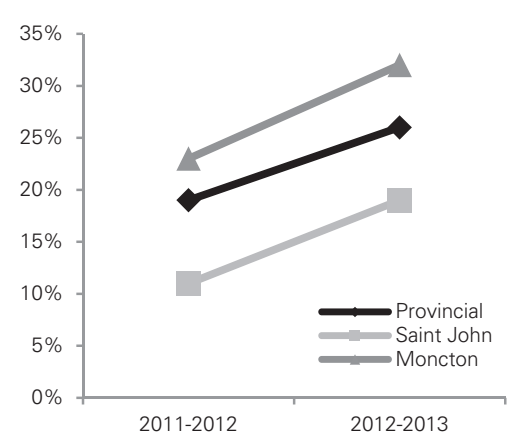

Developing a tool to evaluate trauma team leader performance: initial reliability testing. S. Reid, ${ }^{*}$ A. Coates, ${ }^{\dagger}$ A. Nassar, ${ }^{\dagger}$ F. Farrokhyar, ${ }^{*}$ F. Tuma, ${ }^{\dagger}$ S. Faidi. ${ }^{\dagger}$ From *McMaster University, and the tHamilton Health Sciences Trauma Program, Hamilton, Ont.

Background: Trauma resuscitation is a structured and complex process. As part of training, fellows and residents assume the trauma team leader (TTL) role in trauma resuscitation under the supervision of the attending physician. We are unaware of a validated instrument to assess TTL trainee performance; hence, we developed the Trauma Team Leader (TTL) evaluation form. The aims of this study are to assess the inter-rater reliability of the TTL evaluation form using high-fidelity trauma simulations and to determine the feasibility and utility of the form as it relates to trauma education. Methods: We used a literature review and consultation with clinical experts to design a form to assess clinical competence and interpersonal skills required to lead the resuscitation. Domains include critical assessment, communication and leadership, decision-making, clinical performance, and teach- ing. Five items address each domain. Each item is assessed using a 5 -point Likert scale. Trainees consented to having faculty assessors evaluate their performance during the simulation. An online survey was sent to assessors and trainees to gauge feasibility, practicality and utility of the form. Results: Results of initial reliability testing of our newly created MacTrauma TTL Assessment Tool are encouraging. The proposed tool reached, or nearly reached, substantial agreement for 3 of 4 domains. Conclusion: Preliminary feedback from raters and trainees on the feasibility, practicality and utility of the tool was positive. We propose changes to improve the performance of the tool.

Field trauma triage guidelines as a predictor of key emergency department time intervals. S. Benjamin, A. Chisholm, I. Watson. From the New Brunswick Trauma Program, Horizon Health Network, Saint John, NB

Background: Field trauma triage guidelines (FTTGs) are based on moving the most seriously injured patient directly to the appropriately resourced facility. The New Brunswick Trauma program FTTG identifies step 1A failures as: need for ventilatory support, Glasgow Coma Scale (GCS) $\leq 8$, blood pressure (BP) $<60 \mathrm{~mm} \mathrm{HG}$, and odds ratio (OR) decreased breath sounds with respiratory distress. Step 1 failures were identified as: $\mathrm{GCS}<14$, $\mathrm{BP} \leq 90 \mathrm{~mm} \mathrm{Hg}$; OR respiratory rate under 10 or over $29 \mathrm{~min}-$ utes. It is assumed there would be no difference in the throughput time intervals between these 2 patient subsets. Methods: This retrospective study compared time intervals in the emergency department (ED) for those admitted patients arriving directly to the Saint John Regional Hospital having failed step 1A versus step 1 during the 2012 to 2013 year. Patients were divided into 2 groups: group 1 (step 1A, $n=27$ ) and group 2 (step 1, $n=13$ ). The primary outcomes examined were interval from ED arrival to time of CT, time of ED admission and time of ED departure. Results: Group 1 had shorter mean times from ED arrival to time of CT, time of admission and time of ED departure than group 2 even though group 1 often required immediate critical interventions. Conclusion: Step 1A and 1 failures did show differences across all measured time intervals and may be a predictor of immediate and more intense ED resource requirements.

Vagal nerve stimulation improves coagulopathy in hemorrhagic shock: a thromboelastometric study in an animal model. J. Rezende-Neto, ${ }^{*}$ R. Lage, ${ }^{+}$M. Carvalho Jr., ${ }^{+}$ T. Almeida, ${ }^{\dagger}$ C. Trant, ${ }^{\dagger}$ M. Andrade, ${ }^{\dagger}$ C. Kushmerick, ${ }^{\dagger}$ S. Rizoli, ${ }^{*}$ J. Cunha-Melo. ${ }^{\dagger}$ From *St. Michael's Hospital, Toronto, Ont., and the tUniversidade Federal de Minas Gerais, Belo Horizonte, Brazil

Background: Trauma associated coagulopathy (TAC) in hemorrhagic shock (HS) is multifactorial and inflammation plays a major role. The anti-inflammatory pathway is regulated via the vagus nerve. We hypothesized that vagal nerve stimulation (VNS) can improve coagulopathy through inflammation. Methods: Wistar rats $(n=48)$ were divided in 8 groups: group sham; (G1) VNS w/o HS; (G2A) HS w/o VNS; (G2B) HS followed by VNS; (G3A) VNS followed by HS; (G3B) Bilateral vagal nerve transection followed by HS; (G4A) HS followed by transection and distal stump VNS; and (G4B) HS followed by transection and proximal stump VNS. Hemorrhage (45\% of baseline mean arterial 
pressure $\times 15 \mathrm{~min}$ ) was followed by normotensive resuscitation with Lactated Ringer's. The left vagus nerve was stimulated $(3.5 \mathrm{~mA}, 5 \mathrm{~Hz})$ for 30 seconds 7 times. Samples were obtained at baseline and 60 minutes for thromboelastometry (Rotem $\left.{ }^{\circledR}\right)$ and cytokine assays (IL-1, IL-6, IL-10). Paired $t$ test was used for comparison. Results: Maximum clot firmness (MCF) significantly decreased in G2A after HS $(71.5 \pm 1.5$ v. $64 \pm 1.6, p<0.05)$. The MCF significantly increased in G4B compared with baseline $(67.3 \pm 2.7$ v. $71.5 \pm 1.2, p<0.05)$. G4A also showed significant improvement in Alfa angle, and clot formation time compared with baseline. Group 4A showed a significant decrease in IL-6 and a significant increase in IL-10 $(p<0.05)$. Conclusion: Stimulation of the distal stump of the vagal nerve during resuscitation (G4A) improves coagulation after hemorrhagic shock. An anti-inflammatory mechanism could be involved in this process.

Flat or fat? Inferior vena cava diameter is an accurate marker for occult hypoperfusion in major trauma patients. D. Kim, A. Nguyen, S. Bricker, F. Bongard, A. Neville, B. Putnam, D. Plurad. From Harbor UCLA Medical Center, Los Angeles, Calif.

Background: Identifying occult hypoperfusion $(\mathrm{OH})$ is critical in the management of trauma patients as delayed recognition resulting in worse outcomes. We hypothesized that inferior vena cava (IVC) diameter is a predictor of $\mathrm{OH}$ and is associated with worse outcomes in trauma patients. Methods: We performed a 1-year retrospective analysis of all major trauma team activations at our level 1 trauma centre. Inclusion criteria included adult patients that underwent evaluation with a CT scan of the torso, an arterial blood gas, and lactate. A flat IVC was defined as a transverse to anteroposterior ratio $\geq 2.5$ at the level of the suprarenal IVC. Correlation analysis was performed between IVC ratio and base deficit/lactate. Occult hypoperfusion was defined as a base deficit $\geq 4.0$ in the absence of hypotension (systolic blood pressure $[\mathrm{SBP}] \leq 90)$. Results: Of 264 patients, 52 had a flat IVC. Patients with a flat IVC had a higher Injury Severity Score (ISS), lactate, and base deficit versus those with a fat IVC. Flat IVC patients required greater amounts of fluids $(p<0.04)$ and blood $(p<0.01)$. On multivariate analysis, after controlling for age, sex, comorbidities, mechanism, ISS, and anemia, a flat IVC was independently associated with an increased risk for $\mathrm{OH}(\mathrm{OR} 2.87,95 \%$ CI 1.33-6.17; $p<0.007)$ and overall complications (OR 2.26, 95\% CI 0.99-5.20; $p=0.05$ ). The Receiver Operating Characteristic area under the curve for a flat IVC to detect $\mathrm{OH}$ was 0.7 . Conclusion: A flat IVC is an accurate marker for occult hypoperfusion in major trauma victims. Identification of this radiographic finding may help stratify patients who require more aggressive resuscitation and intensive monitoring.

External genital trauma: isolated injuries or just the tip of the iceberg? D. Kim, A. Nguyen, S. Bricker, F. Bongard, A. Neville, B. Putnam, D. Plurad. From Harbor UCLA Medical Center, Los Angeles, Calif.

Background: Traumatic injuries to the external genitalia are uncommon following trauma. Limited data exist regarding the clinical presentation and outcomes in patients with these injuries. We sought to describe our institutional experience with external genital trauma and to assess the impact of these injuries on shortterm outcomes including sexual dysfunction. Methods: A 10year retrospective analysis of a level 1 trauma centre database was performed to identify patients with external genital injuries. Specific genitourinary injuries, associated injuries, need for operative intervention, and complications including sexual and genitourinary dysfunction were assessed. Results: Of 156 patients, 139 $(89 \%)$ were male and the mean age was $27 \pm 12$ years. Penetrating mechanism of injury accounted for $71 \%$ of all injuries. The most commonly injured structures were the testicles (93 [69\%]), scrotum $(89$ [66\%]), and penis (50 [37\%]). Eighty-three patients $(53 \%)$ sustained associated injuries involving the extremities (49 [31\%]), abdomen (22 [14\%]), and vasculature (21 [13\%]). Seventy-one patients (46\%) required extra-genital invasive procedures. The incidence of in-hospital complications was 6\% (10 of 156). Short-term outpatient follow-up data was complete for 99 patients (64\%). Among this cohort, 18\% reported sexual dysfunction and $30 \%$ of patients had ongoing genitourinary and wound care issues. Conclusion: External genital injuries rarely occur in isolation and short-term complications including sexual dysfunction are common. Improved efforts to follow-up and systematically enquire into sexual and genitourinary health care issues are needed in patients sustaining these injuries.

Isolated vena cava injuries from blunt trauma: a diagnostic dilemma with life-threatening hemorrhage. M. Kim, ${ }^{*}$ L. Jamal, ${ }^{\dagger}$ B. Al-Shareef, ${ }^{*}$ N. Ahmed, ${ }^{*}$ J. Rezende Neto. From the *University of Toronto, Toronto, Ont., †King Abdulaziz University Hospital, Riyadh, Saudi Arabia

Background: Blunt injuries to the abdominal vena cava (IVC) seldom occur in isolation. IVC injury can cause retroperitoneal hemorrhage, not initially detectable by ultrasound (FAST) or peritoneal lavage (DPL). A recent case from our institution prompted us to determine the frequency of isolated blunt IVC injuries, their clinical presentation and management. Methods: A comprehensive OVID Medline literature review resulted in 122 papers regarding IVC injuries. After excluding penetrating trauma and patients with associated abdominal injuries (major vessels and viscera), we compared the clinical circumstances and outcomes of each case, along with our recent patient. Results: Of 1491 cases of IVC injury, 277 (18.8\%) were from blunt trauma. Of these 277 cases, 12 (4.3\%) had isolated IVC trauma without other abdominal organ injury. Seven of the $12(58.3 \%)$ cases presented with hypotension. Only one patient had abdominal free fluid and 9 (75\%) patients underwent CT scan. Nine (75\%) patients underwent surgery while $3(25 \%)$ were managed nonoperatively. All patients presenting with hypotension required surgery. Of all isolated IVC injuries, $83.3 \%$ survived compared with $72 \%$ with any IVC injury. Conclusion: Isolated blunt injury to the IVC is a rare event. The diagnostic dilemma is that many patients present with hypotension but without free fluid. Even after CT, all of these patients needed a laparotomy. Patients with isolated blunt IVC injuries have a better survival than the total population of patients with any IVC injury. An isolated IVC injury should be considered in hypotensive blunt trauma patients without signs of other abdominal injury.

Trauma patients with increased hospital length of stay and rehabilitation facility length of stay are at risk for a 
lower discharge functional independence score. F. Muakkassa, ${ }^{*}$ R. Marley, ${ }^{*}$ S. Horattas, ${ }^{\dagger}$ J. Papas, ${ }^{\dagger}$ A. Salvator, ${ }^{*}$ A. Hayek. ${ }^{*}$ From *Akron General Medical Center, Akron, Ohio, +Northeast Ohio Medical University Rootstown, Ohio, ‡Edwin Shaw Hospital for Rehabilitation, Cuyahoga Falls, Ohio

Background: This study is to determine whether factors from a trauma-related hospitalization discharged to a rehabilitation facility $(\mathrm{RF})$ would impact the discharge functional independence measure (FIM) score. Methods: Data was retrospectively collected from registries from Jan. 1, 2002, to Dec. 31, 2009, matching level I trauma patients discharged to a RF. Data were analyzed using $\chi^{2}$ and $t$ test as well as multivariate logistic regression used to determine factors predicting lowest quartile (18-76) discharge FIM scores (FIMLT76). Results: There were 323 patients excluding patients who died at the RF. The mean age was 50.3 years and $65 \%$ were males. Significant multivariate predictors of FMLT76 included age (OR 1.05, 95\% CI 1.02-1.07; $p<0.000$ ), spinal cord injury (OR 7.22, 95\% CI 2.74-19.03; $p=0.000)$, male sex (OR 0.43, 95\% CI 0.21-0.85; $p=0.01)$, admission FIM (OR 0.93, 95\% CI $0.91-0.95 ; p<0.000)$ and an increased risk (OR 2.20, 95\% CI 1.41-3.45; $p=0.001$ ) was observed with a higher ratio of hospital length of stay (LOS)/RF LOS. Traumatic brain injuries (TBIs) and type of trauma were not significant predictors. Conclusion: As the ratio of hospital LOS to RF LOS increases, the odds of FIMLT76 increases by 2fold adjusting for spinal cord injury, ISS, age, sex and RF admission FIM score. The longer the patients with non-spinal cord or TBIs stay in a hospital before discharge to RF, the more negative impact on their rehabilitation potential and thus decrease their functional outcome. Factors affecting delay in transfer from a hospital to RF should be identified in future studies.

Implementation of a prevention bundle to decrease utis in patients admitted to the trauma service. A. Keenan, H. Knight, M. Waggott, J. Pachal, E. Clark, Y. Renaud. From The Ottawa Hospital, Ottawa, Ont.

Background: Catheter associated urinary tract infections (CAUTI) are one of the most common complications experienced by trauma patients. A 2012 study at our level 1 trauma centre demonstrated $12 \%$ of trauma patients developed a UTI. A program to reduce these infections was implemented. Methods: Data was collected over a 6 month period (July 2013-January 2014) after staff were educated regarding a CAUTI prevention bundle. The need for ongoing catheterization was reviewed daily with nursing staff, with the goal of early removal. Patients were monitored daily for signs of UTI. Data collection focused on location, date and duration of urinary catheter insertion. Also captured were gender, age, ISS, antibiotic use, ICU and hospital length of stay (LOS). Results: 167 trauma patients were admitted over a 6-month period. Seventy-seven percent required catheter insertion; $9.5 \%$ of those developed a CAUTI. A strong association was identified between the length of catheterization and CAUTI incidence. The average number of foley days for patients who developed a CAUTI was 16 days versus 7 days for those with no urinary infection. There was a significant reduction in the duration of catheterization from 2012 to 2013 (16 v. 8 d). Hospital length of stay showed a similar pattern: 35 days for those who developed a CAUTI versus a 16-day average stay if no CAUTI, resulting in significant cost savings. Conclusion: The implementation of a UTI bundle was effective in reducing the number of catheter days and the incidence of CAUTIs in trauma patients. Reducing the length of catheter time decreased the incidence of UTIs. The occurrence of CAUTIs was associated with a longer hospital LOS.

Team-based errors in trauma resuscitation: a systematic review. H. Heipel, M. McGowan, C. Hicks. From St. Michael's Hospital, Toronto, Ont.

Background: Management of multi-system trauma is fraught with challenges owing to complex clinical presentations, time dependency for intervention and ad hoc assembly of the trauma teams. Despite the creation of Advanced Trauma Life Support (ATLS) and dedicated trauma centres, team-based medical error occurs in the majority of trauma resuscitation. Methods: A systematic review of medical mistakes and trauma among pediatrics and adults was conducted with a primary search of Medline, Embase, Web of Science and Cochrane databases for studies after 1980 (ATLS implementation) with the terms "human factors," "wounds and injuries," "medical errors," "trauma centers" and "emergency medicine"; non-English articles, case studies, editorials reviews, and letters were excluded. A secondary search of bibliography lists, Google Scholar served to increase sensitivity and rigor. Two independent reviewers assessed titles and abstracts to identify suitable articles based on a priori criteria. Disagreement was resolved by consensus by a third party. Medical error type was defined, categorized, and recorded on a priori standardized form. Results: The search returned 893 citations. Agreement was moderate for title $(\kappa=0.65)$ and abstract $(\kappa=0.6)$; with 11 articles meeting inclusion and selected for text review. Of these, 2 articles met full inclusion criteria. The first paper was a video registration incorporating 1256 patients and 387 team performance analysis over 2 years. Authors concluded that there was a significant reduction of errors with evidence of adequate prehospital report, effective leadership, efficient leadership, evident leader, and team organization $(p<0.05)$. The second paper included a chart review of 764 patients over 5 years using Joint Commission on Accreditation of Healthcare Organizations error taxonomy as a measure to analyze team errors. These authors found that human errors predominated contributing to potential preventable and preventable deaths and stated that the majority of preventable errors were because of the complex trauma team incorporating many specialties and level of experience. Bibliography search revealed 20 suitable articles. Qualitative data analysis is currently underway of the selected hand articles. Conclusion: A systematic review of the literature on team-based errors in trauma, paired with institutional quality and safety data may provide powerful data to inform the development of simulation-based team training interventions, with the specific goal of improving patient safety and mitigating the impact of consequential medical error.

Current use of live tissue training in trauma: a descriptive systematic review. L. Teodoro da Luz, S. Vlachos, B. Nascimento, A. Nathens, H. Tien. From the Sunnybrook Health Sciences Centre, Toronto, Ont.

Background: Training in trauma uses combinations of didactics, computer simulations, manikins, task trainers, moulage scenarios 
and live tissue training (LTT). Live tissue training might be considered as gold standard in certain forms of training; however, concerns for animal welfare, advances in computerized simulation, educational considerations, and economic barriers have drawn a critical eye to animal use. There is no definitive evidence in the literature about the use of LTT in trauma. We conducted a systematic review to determine the value of LTT alone or compared with other simulation training methods in trauma. Methods: We considered observational studies and randomized controlled trials (RCTs) on Medline, Embase and Cochrane, that examined LTT alone or studies comparing LTT to other methods. We extracted data on demographics, design, setting, participants, types of LTT and simulation, intervention, outcome measures and main findings. We assessed methodological quality using the Newcastle-Ottawa scale for cohort studies and the risk of bias for RCTs with the Cochrane Collaboration tool. Independently and in duplicate, we adjudicated studies for inclusion and data abstraction. Results: Seventeen studies $(n=2718)$ met inclusion criteria. There were 3 RCTs and 14 prospective cohorts. Anesthetized swine were used in 11 studies, and dogs, goats and sheep in 1 study, respectively. Three studies did not report details about the choice of LTT. Live tissue was used without a comparator in 7 studies. Other 6 studies, to different degrees, used LTT and other methods but did not conduct any comparisons. Finally, 4 studies conducted comparisons between LTT and other methods. Seven studies used HPS, 2 studies used cadavers, 2 studies computer simulation, 2 studies video presentations, and 1 study used moulage. The overall methodological quality was low. Conclusion: There is limited evidence supporting the superiority of LTT when compared with other types of simulation. Data on training effects of LTT versus other simulation methods on outcomes are lacking.

Myth busting: current surgical beliefs about gunshot wounds. S. Hafertepen, J. Davis, R. Townsend, L. Sue, K. Cagle. From the University of California San Francisco - Fresno, Fresno, Calif.

Background: Poorly designed experiments and popular media have led to a number of myths about wound ballistics. Some myths have been incorporated into the trauma literature, including the Advanced Trauma Life Support (ATLS) course. We hypothesized that these erroneous beliefs would be prevalent, even among those providing care for patients with gunshot wounds (GSWs), but could be addressed through education. Methods: A critical review of the wound ballistics literature, including ATLS, was performed and several myths identified. These myths included wounding mechanism and characteristics, lead poisoning, débridement, and antibiotic use. A pretest, 40minute lecture on wound ballistics, and a post-test were developed. Subsequently surgery and emergency medicine services in 3 separate cities were studied. All 3 sites were busy, urban trauma centres with a significant amount penetrating trauma. The preand post-test scores were compared and correlated with demographic data including ATLS course completion, firearm/ ballistics experience, years of post-graduate medical experience. Results: In all, 115 clinicians participated in the study. Average pretest score of $34 \%$ improved to $78 \%$ on the post-test and there were improvements in all areas of knowledge $(p<0.001)$. Years of post-graduate medical experience correlated with higher pretest score $(p=0.021)$; however, ATLS status did not $(p=0.774)$. Conclusion: Erroneous beliefs about wound ballistics are prevalent among clinicians who commonly treat victims of GSWs. These beliefs could lead to inappropriate treatment. Focused education markedly improved knowledge. The current ATLS course and manual promulgate some of these myths and should to be revised.

Clinical manifestations of tension pneumothorax: a systematic review and meta-analysis. D. Roberts, ${ }^{*}$ S. Leigh-Smith, ${ }^{\dagger}$ P. Faris, ${ }^{\neq}$C. Ball, ${ }^{\delta}$ H. Lee Robertson, ${ }^{\delta}$ C. Blackmore, ${ }^{*}$ E. Dixon, ${ }^{\delta}$ A. Kirkpatrick, ${ }^{\S}$ J. Kortbeek, ${ }^{*}$ H.T. Stelfox. ${ }^{*}$ From the *University of Calgary, Calgary, Alta., †NHS, Edinburgh, UK, ¥Alberta Health Services, and §Foothills Medical Centre and University of Calgary, Calgary, Alta.

Background: Although the clinical manifestations of tension pneumothorax (TPTX) have been suggested to differ among subjects of varying respiratory status, it remains unknown if these differences are supported by evidence. Methods: We searched electronic bibliographic databases (1950-Oct. 15, 2013), review articles, and included article references for case reports/series and observational studies reporting original data on the clinical manifestations of TPTX. Identified data were summarized using formal narrative synthesis techniques alongside a meta-analysis of case report/series data using multivariate generalized estimating equations (GEE). Results: Among 3184 articles identified, we included 160 case series/reports (186 TPTX patients, median age $46 \mathrm{yr}$ IQR 27-61) and 5 observational studies. Although hypoxia, tachycardia, ipsilateral decreased air entry and percussion hyperresonance were commonly reported TPTX clinical manifestations in case reports/series, jugular venous distention and tracheal deviation were uncommonly reported. Using GEE, the adjusted presenting partial pressure of arterial oxygen/fraction of inspired oxygen ratio was similar between TPTX patients of varying respiratory status $(p=0.54)$. In contrast, the adjusted odds of hypotension and the presenting mean arterial blood pressure was 8.6 (95\% CI 1.6-44.7) times higher and $43.0 \mathrm{~mm} \mathrm{Hg}$ (95\% CI 21.9$64.0 \mathrm{~mm} \mathrm{Hg}$ ) lower among patients receiving Positive Pressure Ventilation as compared with those who were breathing spontaneously, respectively. These findings were supported by the narrative synthesis of observational study results. Conclusion: Our results support the findings of animal studies that spontaneously breathing subjects with a TPTX frequently develop respiratory signs and symptoms without hemodynamic compromise. A revised list of clinical criteria stratified by patient ventilatory status is suggested.

Unexpected survivors in trauma: Where do brightspots occur? E. Wong, ${ }^{*}$ X. Hui, ${ }^{+}$W. Leeper, ${ }^{\dagger}$ D. Deckelbaum, ${ }^{*}$ T. Razek, ${ }^{*}$ E. Haut. ${ }^{\dagger}$ From ${ }^{*}$ McGill University Health Centre, Montréal, Que., and the TJohns Hopkins University School of Medicine, Baltimore, Md.

Background: Benchmarking of trauma care quality is a critical step to help improve outcomes. Mortality is frequently predicted using the Trauma and Injury Severity Score (TRISS) but unexpected survival still occurs in severely injured patients. We sought to determine if unexpected survivors cluster at specific trauma 
centres in order to plan future quality improvement initiatives. Methods: Data from all adult ( $\geq 16 \mathrm{yr}$ ) patients with blunt or penetrating injury, treated at a level 1 trauma centre from 2007 to 2011 and with a probability of survival of less than $10 \%$ as predicted by TRISS were extracted from the National Trauma Data Bank (NTDB). Multivariate logistic regression analysis with mortality as the outcome was performed to obtain risk-adjusted observed-to-expected $(\mathrm{O} / \mathrm{E})$ mortality ratios for each centre based on the Trauma Quality Improvement Program (TQIP) methodology. Results: Of a total of 3313117 patients in the NTDB, 22449 from 183 level 1 trauma centres met our inclusion criteria. The figure shows a caterpillar plot of risk-adjusted O/E mortality ratios $(95 \% \mathrm{CI})$ from these centres. Fifteen facilities were identified as high performers and 61 were low performers. Conclusion: Unexpected survivors of severe injuries tend to cluster at specific trauma centres. Future quality improvement initiatives could study these centres to determine structures or processes of care that lead to better outcomes.

A simulation-based trauma education program: Does it improve trauma management? T. Chaplin. From Kingston General Hospital, Kingston, Ont.

Background: There is a growing literature base to support the use of human patient simulators in trauma education. The purpose of this study was to evaluate the impact of an 11-month simulation-based trauma education program (STEP) on the trauma management skills of senior medical residents in the simulation laboratory and the real trauma room.. Methods: Two distinct cohorts of post-graduate year (PGY)-3 residents participated in this study. The first cohort, the control group, consisted of 14 PGY-3 residents who only took part in the evaluation process. The second cohort, the intervention group, consisted of 28 PGY-3 residents who participated in the 11-month STEP and then took part in the same evaluation process. This process consisted of form-based evaluations (both self-evaluations, and staff evaluations), as well as an evaluation on a video-recorded standardized simulated trauma scenario. Results: The crisis resource management (CRM) scores on the standardized simulated trauma scenario were not significantly different between the 2 groups. The intervention group scored higher on the staff evaluations across all categories: overall score $(5.0 \mathrm{v}$. 4.7$)$, knowledge $(5.1 \mathrm{v}$. 4.9), leadership (5.3 v. 4.6), problem solving (5.2 v. 4.7), situational awareness (5.3 v. 4.7), resource utilization $(5.4 \mathrm{v} 4.7)$, or communication (5.4 v. 4.8). The intervention group scored higher on the self-evaluations in all categories. Conclusion: Our present study did not find any differences in the management of a standardized trauma scenario by PGY-3 residents following our simulation-based trauma education program. Secondary outcomes demonstrate a trend of increasing CRM scores and selfevaluation scores.

Effect of 1 (red blood cell): 1 (plasma) transfusion on coagulation of severely injured patients. B. Nascimento, ${ }^{*}$ L. Luz, ${ }^{*}$ J. Callum, ${ }^{*}$ H. Tien, ${ }^{*}$ S. Rizoli. ${ }^{+}$From *Sunnybrook Health Sciences Centre and TSt. Michael's Hospital, Toronto, Ont.

Background: It is unclear whether the potential benefit of aggressive plasma transfusion at a near 1:1 ratio with red blood cell (RBC) relates to improved coagulation via correction of clotting factor deficits. We recently completed a feasibility randomized clinical trial (RCT) on a 1:1:1 transfusion versus the laboratoryguided standard of care transfusion. Using data from this RCT, we evaluate changes in standard and special coagulation assays at the end of study interventions. Methods: Subgroup analysis of a RCT. We included severely injured bleeding patients with blood samples collected immediately at the initiation and termination of study protocols. The main outcome was the impact of transfusion protocols on coagulation, measured by the increase of clotting factor activity from baseline to end of study intervention in both $\operatorname{arms}(\Delta=[\mathrm{X} 2 / \mathrm{X} 1]-1)$. The proportion of patients with normalized ( $\geq$ protocol termination in both study groups were evaluated. $\chi^{2}$ statistics, Student $t$ test and Wilcoxon rank-sum test were used when appropriate. Results: 1:1 transfusion produced statistically significant increases in the following coagulation factors, as compared with control: factor II $(0.45[0.1 ; 0.84] \mathrm{U} / \mathrm{mL}$ v. 0.08 $[-0.11 ; 0.38] \mathrm{U} / \mathrm{mL} ; p=0.044)$; factor IX $(0.25[0.01 ; 0.68] \mathrm{U} / \mathrm{mL}$ v. $-0.01[-0.11 ; 0.26] \mathrm{U} / \mathrm{mL} ; p=0.028)$; factor $\mathrm{X}(0.29$ [0.12; 0.74$]$ $\mathrm{U} / \mathrm{mL}$ v. $0.04[-0.16 ; 0.22] \mathrm{U} / \mathrm{mL} ; p=0.023)$; and factor XI $(0.59$ [0.17-1.94] U/mL v. $0.05[-0.2 ; 0.54] \mathrm{U} / \mathrm{mL} ; p=0.018)$. Conclusion: 1:1 transfusion protocol was superior to standard laboratory-guided transfusion in correcting clotting factor deficits in severely injured bleeding patients.

Indications for damage control surgery in adult civilian trauma patients: an international scoping study. D. Roberts, ${ }^{*}$ N. Bobrovitz, ${ }^{*}$ D. Zygun, ${ }^{\dagger}$ A. Kirkpatrick, ${ }^{\dagger}$ C. Ball, ${ }^{\dagger}$ P. Faris, ${ }^{\ddagger}$ H.T. Stelfox. ${ }^{*}$ From the ${ }^{*}$ University of Calgary, tFoothills Medical Centre and University of Calgary, and $¥$ Alberta Health Services, Calgary, Alta.

Background: Although damage control surgery (DCS) may decrease mortality risk among the severely injured, its use is associated with increased morbidity when inappropriately applied. We sought to systematically scope the DCS literature, identify its proposed indications, and examine the extent and nature of available evidence. Methods: We searched electronic databases (Medline, PubMed, Embase, Scopus, Web of Science, and the Cochrane Database), included article bibliographies, and conference proceedings. Articles involving civilian trauma patients that proposed 1 or more indications for DCS were included. Results: Among 16501 identified citations, we included 124 articles in the scoping study. Of these, 74 (59.7\%) represented original research, primarily retrospective and prospective cohort studies, while 48 (38.7\%) were narrative reviews and 2 (1.6\%) were guidelines. A total of 52 preoperative, including 3 prehospital and 25 emergency department, and 385 intraoperative indications for DCS were identified. These frequently represented characteristics of the injured patient $(94.5 \%)$, surgeon $(18.6 \%)$, health care team $(0.5 \%)$, trauma care process $(18.1 \%)$, and/or trauma care structure or environment (3.4\%). Among patient-based indications, $55.4 \%, 59.2 \%$, and $13.5 \%$ were physiology-, resuscitation-, and/or injury-based, respectively. Injury-specific indications were commonly associated with trauma to the abdomen $(79.2 \%)$ or thorax $(9.6 \%)$ and with the potential for massive hemorrhage (56.9\%). Conclusion: Although a large number of DCS indications have been proposed, most are suggested to be applied in the operating room and are based on characteristics of the physiology or injury complex of the patient. There is a need to develop 
objective, evidence-based indications for the appropriate use of DCS.

Excess of osteoarthritis development following fracture or dislocation of lower limb joints. J. Vaillancourt, J. Sampalis. From JSS Medical Research and McGill University, Montréal, Que.

Background: To assess the excess of osteoarthritis (OA) development following fracture or dislocation of lower limb joints in the population of the province of Quebec, Canada. Methods: Quebeckers aged 18-85 years who had a first traumatic fracture or dislocation of the hip, knee or ankle in 1998 were identified from the Quebec Trauma Registry. The reference population included individuals who did not have any fracture or dislocation of the lower limb joints and were randomly selected from Med-Echo hospitalization database on a 1:1 ratio based on age, gender and year of discharge of the trauma cohort. Individuals with musculoskeletal disease history were excluded. Individuals were followed until December 2003. OA events were identified by the ICD-9 codes. Standardized OA incidence rates (SMRs) and 95\% confidence intervals were calculated by age categories at trauma occurrence. Results: Of the 1281 trauma individuals, 745 (58.2\%), 89 (6.9\%) and 447 (34.9\%) had a hip, knee and ankle trauma, respectively. The mean (standard deviation) age of the trauma cohort and the reference population was 58.23 (SD 18.5) and 60.03 (SD 19.6) years, respectively, with $54.9 \%$ females. Younger adults aged 18-34 years at trauma occurrence had an SMR of 8.62 (95\% CI 5.79-12.88). Individuals aged 35-54 years, 55-74 years and 75-85 years at trauma occurrence had respectively significant excess of OA development of $10 \%, 32 \%$ and $23 \%$ relative to the reference population. Conclusion: There is significant excess of OA development following fracture or dislocation of the lower limb joints, especially among younger adults aged less than 35 years at trauma occurrence.

Getting closure in abdomens that won't close. D. Roizblatt, ${ }^{*}$ J. Al-Oweis, ${ }^{*}$ S. Iqbal, ${ }^{+}$A. Babsail, ${ }^{+}$ M. Alhabboubi, ${ }^{*}$ M. Elhusseini, ${ }^{*}$ P. Fata, ${ }^{*}$ T. Chughtai, ${ }^{*}$ C. Malo, ${ }^{\dagger}$ D. Deckelbaum, ${ }^{*}$ T. Razek, ${ }^{*}$ K. Khwaja. ${ }^{*}$ From the *McGill University Health Centre, Montréal, Que., and the †Montréal General Hospital, Montréal, Que.

Background: Inability to close fascia in patients with an open abdomen (OA) is a recurrent problem after frequent take backs to the OR. When fascia is deemed "unclosable," skin only can be closed, or in certain cases allow for granulation with skin grafting. Our objective was to describe these 2 options when fascia cannot be closed. Methods: Retrospective study conducted on OA patients admitted to a level 1 trauma centre from January 2010 to March 2013. Patients surviving $\geq 15$ days after first surgery and with inability for fascial closure were included. Demographics and standard outcome variables were recorded for patients undergoing skin closure (SC) and granulation with split-thickness skin graft (SG). Univariate analysis was performed. Results: Sixtyfour patients with an OA were identified, with 24 patients not achieving fascial closure. Of these 24, 20 survived 15 days or more. Eleven (55\%) patients had SC; 9 (45\%) had SG. Age, sex and APACHE II showed no statistical difference between groups. Median number of take backs to OR did not differ between groups. Infectious complications (abscess, pneumonia, sepsis) were similar in both groups. Median intensive care unit (ICU) length of stay (LOS) and ventilation days were 22 and 18.3 days respectively for the SC group compared with 31 and 39.8 days, respectively, in the $\mathrm{SG}$ group $(p=0.038$ and $p=0.033)$. Conclusion: In patients whose fascia cannot be closed, early skin closure should be attempted. The alternative of allowing granulation and skin grafting leads to significantly longer ventilation and ICU LOS. Further work is needed to determine at what point the fascia should be considered "unclosable" and skin closure should be done.

Nutritional assessment of open abdomen patients. M. Elhusseini Hassan, ${ }^{*}$ S. Iqbal, ${ }^{\dagger}$ M. Alhabboubi, ${ }^{*}$ D. Deckelbaum, ${ }^{*}$ D. Roizblatt, ${ }^{*}$ J. Al-Oweis, ${ }^{*}$ L. St. Laurent, ${ }^{*}$ E. Eckert, ${ }^{*}$ D. Bracco, ${ }^{*}$ T. Razek, ${ }^{*}$ K. Khwaja. ${ }^{*}$ From the *McGill University Health Centre, and the TMontréal General Hospital, Montréal, Que.

Background: The use of the open abdomen (OA) procedure as a surgical option has increased in recent years. Optimizing the nutritional needs continues to pose a challenge for surgeons. Our objective was to determine these critically ill patients were adequately fed comparing our practice with the Penn State equation. Methods: A retrospective study was conducted on 24 patients managed with an OA for at least 7 days admitted at a level 1 trauma centre between January 2010 and March 2013. Daily caloric intake was measured by tabulating the total enteral and/or parenteral nutrition received, as well as the total relevant fluid and medication infusions. Patient demographics and standard outcome variables were also recorded. The nutritional target using the Penn State equation was calculated for each patient. Results: The median age was 41 years. At 7 days, $4.1 \%$ and $8.3 \%$ of patients met over $90 \%$ of their target calories and protein, respectively. At 10 days, 50\% reached the caloric target, while $41.6 \%$ achieved their protein target. At 14 days, 59\% achieved target protein intake while $27 \%$ achieved the caloric target. Patients with higher body mass index were less likely to achieve their caloric needs at 10 days $(p=0.04)$. Unadjusted survival was higher in the group that achieved target protein needs at 14 days $(p=0.03)$. Conclusion: Based on the Penn state equation, OA patients were insufficiently fed during their intensive care unit stay. Patients who achieved their protein target at 14 days were more likely to survive. Further studies are needed to identify the impact of underfeeding in the OA patients.

Does timing of tracheostomy affect outcome of open abdomen patients? D. Roizblatt, ${ }^{*}$ J. Al-Oweis, ${ }^{*}$ M. Alhabboubi, ${ }^{*}$ S. Iqbal, ${ }^{\dagger}$ C. Malo, ${ }^{*}$ B. Al Bader, ${ }^{*}$ A. Alkhunaizi, ${ }^{\dagger}$ M. Elhusseini, ${ }^{*}$ A. Babsail, ${ }^{*}$ T. Chughtai, ${ }^{*}$ D. Bracco, ${ }^{*}$ D. Deckelbaum, ${ }^{*}$ T. Razek, ${ }^{*}$ K. Khwaja. ${ }^{*}$ From the *McGill University Health Centre, and the tMontréal General Hospital, Montréal, Que.

Background: Benefits of early tracheostomy (ET) have been discussed in the literature, describing fewer intensive care unit days and earlier weaning from the ventilator. Our objective was to assess the benefits of performing ET in patients with an open abdomen (OA). Methods: Retrospective study conducted on OA patients admitted to a level 1 trauma centre from January 2010 to 
March 2013. Tracheostomized patients were grouped into early group (EG), done at or under 10 days after first OA surgery, and late group (LG) done more than 10 days after first OA. Univariate analysis and Cox regression were used to analyze the data. Results: Of 64 total OA patients, tracheosotmy was performed on $27(42.1 \%)$. The EG had 13 (48.1\%) patients while the LG had $14(51.8 \%)$ patients. Univariate analysis is shown in the Table below. Patients undergoing early tracheostomy had significantly lower ventilation days than the late group $(p=0.025)$. Early tracheostomy had a hazard ratio of 9.93 (95\% CI 2.2-45.3) with respect to early weaning from the ventilator. Conclusion: Performing early tracheostomy $(\leq 10 \mathrm{~d})$ in patients with OA has the benefit of early weaning from the ventilator. Furthermore, an early tracheostomy allows patients to be off the ventilator between returns to the operating room or abdominal dressing changes.

\begin{tabular}{lccc} 
Table. & \multicolumn{2}{c}{} \\
\cline { 2 - 3 } & \multicolumn{2}{c}{ Group; no or $\%$} & \\
\cline { 2 - 3 } & Early group & Late group & p value \\
\hline Factor & 35 & 28 & 0.650 \\
\hline Age, yr & $92.3 \%$ & $92.3 \%$ & 0.742 \\
\hline Sex, male & 34.5 & 38 & 0.88 \\
\hline Injury Severity Score & 27 & 27 & 0.35 \\
\hline APACHE II & 76 & 76.5 & 0.583 \\
\hline Length of stay & 27 & 38.5 & 0.185 \\
\hline Days in ICU & 18.4 & $28.6 \%$ & $0.025 *$ \\
\hline Days on ventilator & $46.2 \%$ & $7.1 \%$ & 0.763 \\
\hline Fascial closure & $7.6 \%$ & & \\
\hline Mortality & & & \\
\hline $\begin{array}{l}\text { APACHE II = Acute Physiology and Chronic Health Evaluation II; ICU } \\
\text { unit. } \\
\text { *Significant } p \text { intensive care }\end{array}$ & & & \\
\hline
\end{tabular}

Presence of a traumatic brain injury is not an independent predictor of acute coagulopathy in trauma patients. D. Paskar, ${ }^{*}$ J. R. Neto, ${ }^{\dagger}$ S. Scarpelini, ${ }^{\neq}$B. Nascimento, ${ }^{\delta}$ S. Rizoli. ${ }^{+}$From *Western University, London, Ont., tSt. Michael's Hospital, Toronto, Ont., ¥Faculdade de Medicina da Universidade de Sao Paulo, Ribeirao Preto, Brazil, and the §Sunnybrook Health Sciences Centre, Toronto, Ont.

Background: Previous studies suggest that traumatic brain injuries (TBIs) may independently trigger early trauma coagulopathy (ETC), given the high ETC incidence in patients with isolated TBIs. Most studies, however, have significant limitations and the association between TBI and ETC remains unproven. There is strong evidence that shock and tissue injury are key ETC triggers. This study evaluated whether TBI independently triggers ETC in a large cohort of trauma patients. Methods: Prospective data from consecutive adult trauma patients admitted to a Canadian level 1 trauma centre in 2008 to 2009 with admission thromboelastography (TEG) were included. Patients were initially classified as having isolated TBI (Abbreviated Injury Scale [AIS] head $\geq 2$ + non-head $<2$ ); no TBI (AIS head $<2$ ); or having combined injuries. Early trauma coagulopathy was defined as abnormal admission TEG. Multinomial linear regression analyses were then used to predict coagulation parameters (international normalized ratio, platelet count and TEG) and blood product requirements for 24 hours post-injury. The $95 \%$ confidence inter- vals determined statistical significance. Results: A total of 628 patients ( $72 \%$ male, mean age $39.9 \mathrm{yr}, 83.8 \%$ blunt mechanism) were analyzed. Age, sex and admission physiologic parameters were similar in all groups while Injury Severity Score (ISS) was higher in the combined group. Patients with combined injuries had more ETC and required more blood transfusions than those with isolated TBI. Regression analysis revealed shock $(\mathrm{pH}<7.3)$ and tissue destruction (ISS) were significantly associated with ETC and blood transfusion, while TBI was not. Conclusion: The amount of tissue destruction (ISS) appears to trigger ETC. While TBI is an important cause of morbidity and mortality in trauma, but does not seem to initiate or worsen ETC.

Is infrastructure really the big problem? A trauma verification program at the national referral hospital in Kenya. A. Saleh, J. Hogan, L. Rowe, H. Hawes, J. Kromm. From Innovative Canadians for Change, University of Alberta, Edmonton, Alta.

Background: Trauma and injury are the leading causes of death in the first 4 decades of life globally, exceeding the burden of HIV, tuberculosis and malaria combined, with $90 \%$ of these deaths occurring in low- to middle-income countries (LMICs). Barriers to addressing this burden appear to be lack of infrastructure and poor training. While Kenya has 6947 health facilities including referral hospitals and trauma centres, it lacks a cohesive framework to guide a national trauma program. The facilities also lack the proper systems for trauma care despite the availability of adequate infrastructure, making them inefficient and unable to respond to the immense trauma burden they face. Methods: At the request of the Kenyatta National Hospital (KNH) surgeons and administrators, literature review was performed focusing on various tools and metrics used to perform a Trauma Verification Program globally. The Australasian Trauma Verification Guide was found to be the most appropriate for an LMIC context given it included infrastructure parameters in the evaluation. The program consisted of working with surgeons, disaster management experts, emergency physicians, nurses, and administrators during 2 sets of visits, a preliminary assessment, followed by a comprehensive one. Results: An assessment document identifying gaps in trauma care delivery was collaboratively compiled and shared identifying 20 key recommendations and potential areas for fruitful interventions. Conclusion: A trauma verification process undertaken in Kenya at the national referral hospital, $\mathrm{KNH}$, revealed the deficiencies to be primarily in the systems for trauma care rather than the infrastructure.

Development of the Kenya-University of Alberta orthopedic trauma assessment (KUOTA) tool. K. Almansoori, ${ }^{*}$ V. Mutiso, ${ }^{\dagger}$ A. Saleh, ${ }^{\neq}$J. Hogan, ${ }^{\neq}$H. Hawes, ${ }^{\neq}$J. Kromm, ${ }^{\neq}$ M. Menon. ${ }^{*}$ From the *University of Alberta, Edmonton, Alta., †Kenyatta National Hospital, Nairobi, Kenya, and ‡Innovative Canadians for Change/University of Alberta, Edmonton, Alta.

Background: The University of Alberta's (UA) Office of International Surgery and the non-profit organization Innovative Canadians for Change (ICChange), have been collaborating with local stakeholders to develop a national trauma system in Kenya. A comprehensive trauma systems assessment had demonstrated 
opportunities for improvement in orthopedic trauma services (OTS) at Kenya's largest trauma hospital, the Kenyatta National Hospital (KNH). A review of the medical literature revealed that apart from outcome-indicators, no evidence-based tools specific to orthopaedic trauma-care were available. Therefore, we have developed a novel assessment tool named KUOTA for the identification of major deficiencies in standards of orthopedic care. Methods: The tool's methodology is based on retrospectively collecting a set of 34 clinical indicators to evaluate the care pathway of the 9 most common orthopedic injuries (e.g., femoral shaft fractures), which together compromise approximately $70 \%$ of OTS in-hospital procedures. The indicators (e.g., femoral shaft stabilization $<6 \mathrm{~h}$ or dislocated hip reduction $<6 \mathrm{~h}$ ) were developed from a Donabedian model using Groene's criteria for health care evaluation and content experts to provide evidencebased orthopedic guidelines via a Delphi approach. Results: Preliminary assessment results have demonstrated considerable deviations from published OTS standards, including an average 63 day length of stay for patients with isolated femoral shaft fractures, compared with 3.9 days from North American literature reports. In addition to $\mathrm{KNH}$, the KUOTA tool is currently being applied at the UA hospital, Canada's third largest trauma centre, for both comparison and validation. Conclusion: The KUOTA tool can provide health care administrators with a criteriareferenced method for evaluating and identifying outliers in the care-path of orthopedic trauma-care standards.

Use of geographic information systems to improve understanding of trauma health care infrastructure in a $50 \mathrm{~km}$ radius around Eldoret, Kenya. A. Saleh, ${ }^{*}$ J. Hogan, ${ }^{*}$ J. Kromm, ${ }^{*}$ A. Noor, ${ }^{\dagger}$ H. Hawes. ${ }^{*}$ From *Innovative Canadians for Change, University of Alberta, Edmonton, Alta., †KEMRI Wellcome Trust, Kilifi, Kenya

Background: The World Health Organization (WHO) estimates that trauma causes 5 million deaths annually worldwide, with $90 \%$ of these deaths occurring in low- to middle-income countries. Both a lack of infrastructure or poor trauma systems design could account for this inequity. Injured patients may not have access to timely trauma care due to delays in reaching a limited number of hospitals, or may bypass suitable centres en route to large, overburdened referral hospitals. An improved understanding of the available infrastructure and their levels of care could help decentralize trauma care away from the tertiary centres. Methods: The Kenya Master Health Facilities list and geospatial coordinates for clinics in a $50 \mathrm{~km}$ radius around a tertiary care hospital, Moi Teaching and Referral Hospital (MTRH), in Eldoret, Kenya, were used to plot a geographic information system (GIS). A preliminary trauma assessment process was undertaken at MTRH to clarify the severity of traumas seen, lengths of stay per patient, and support MTRH offers to referral centres. Results: Kenya has 6947 health facilities in total; 301 are located within a $50 \mathrm{~km}$ radius of MTRH. A GIS of clinics and care level around a trauma hospital was plotted. An infrastructure assessment metric of minimal facility readiness requirements was developed based on WHO templates. These results will guide clinic selection for trauma education and training to facilitate decentralization of trauma care. Conclusion: It appears that improving trauma systems, rather than adding infrastructure, is necessary to improve access to effective trauma care in this region.
Rural trauma: Do interhospital transfers affect outcomes? M. Younus, C. Wargo, J. Dove, D. Torres, K. Widom, D. Leonard, S. Baro, J. Blansfield, M.M. Shabahang, J.L. Wild. From the Geisinger Medical Center, Danville, Pa.

Background: In rural Pennsylvania, one regional level 1 trauma centre receives $40 \%$ of its trauma patients as interhospital transfers (IHTs). The aim of this study was to evaluate the outcomes of direct admissions (DAs) versus IHT in a rural setting. Methods: This was a retrospective review of trauma database between January 2008 and December 2012 for transport time, Injury Severity Score (ISS), Abbreviated Injury Scale score, intensive care unit (ICU) length of stay (LOS), hospital LOS, morbidity, mortality, discharge destination and functional outcomes. Results: Out of 6118 total patients, $59.5 \%$ were DA and $40.5 \%$ were IHT. Overall, ISS was similar between the 2 groups but Glasgow Coma Scale score was significantly lower $(p<0.001)$ in the DA group. Head and thorax was the most common region injured in both groups. Mean number of ventilator days and ICU days were significantly higher in the DA but overall hospital LOS, complication rate and in-hospital mortality were not significantly different between the 2 groups. The DA patients with ISS $\geq 15$ had statistically higher ISS, lower GCS, and higher LOS and mortality. However, when stratified by ISS into groups, there were no differences in outcomes. The average time from scene to trauma centre was 1:37 \pm 1:59 [hh:mm] and 6:05 \pm 4:04 [hh:mm] for DA and IHT, respectively. Discharge destination from hospital and functional outcomes were similar between the 2 groups. Conclusion: In rural trauma, IHTs appeared to not have any detrimental impact on outcomes. This may be due to effective triage practices at community hospitals, standardization of care with the practice of Advanced Trauma Life Support guidelines, and the low incidence of penetrating and operative trauma in many rural areas.

\section{Preliminary testing of a novel tablet-based tool for prospective injury surveillance at Groote Schuur Hospi- tal, Cape Town, South Africa. E. Zargaran, M. Hameed. From Trauma Services, Vancouver General Hospital, Van- couver, BC}

Background: Injury surveillance has largely been prohibitive in developing countries secondary to overwhelming volumes and high-costs of creating accurate up-to-date trauma registries. We explored the feasibility of using a newly developed Web-based tablet application, the electronic Trauma Health Record (eTHR), for improving injury surveillance. The eTHR has been designed with capabilities of creating an electronic admission record, operative note, and discharge summary while simultaneously capturing data for injury surveillance when used simply as a clinical tool. Methods: Injury surveillance data was prospectively collected at a level I trauma centre in Cape Town, South Africa. One hundred high-acuity patients with clearly defined admission criteria were captured. Results: Use of eTHR as a clinical charting tool allowed for the instantaneous creation of a real-time self-populating trauma registry. Data entry was found to be complete and accurate, and secure data transfer to a protected database was successful. With geographic information systems, real-time maps of the incidence of traumatic events and flow of patients within the Cape Town trauma system were generated. Males aged 30, and females of 
32 years of age were the most commonly injured. The top 3 mechanisms of injury were penetrating injuries (49\%), blunt assaults (26\%), and motor vehicle crashes (19\%). Issues were found related to connectivity to a strong cellular signal, and with stability data storage on local servers. Conclusion: This preliminary pilot study has shown it is feasible to replace traditional paper-based trauma documentation with an electronic version that has also been programed to create a prospective trauma registry simultaneously. If future implementation is successful with clinicians using eTHR for patient charting, a novel means of seamless and affordable injury surveillance for stronger evidenced-based injury prevention initiatives with no hospital workflow hindrance will be validated.

The iTClamp ${ }^{\mathrm{TM}} \mathbf{5 0}$ is safe and effective in the hands of SRCA EMS providers in the kingdom of Saudi Arabia. K. Abu Haimed, ${ }^{*}$ K. Mottet, ${ }^{*}$ J. McKee, ${ }^{\dagger}$ D. Filips, ${ }^{*}$ S. Al-Turki. ${ }^{*}$ From *Innovative Trauma Care and the +University of Alberta, Edmonton, Alta.

Background: The purpose of this study is to evaluate the safety and effectiveness of the iTClamp 50 when used by Saudi Red Crescent Authority (SRCA) emergency medical service providers to control external bleeding in the pre-hospital setting. The iTClamp 50 is a temporary wound closure device that seals the edges of a wound closed to create a pressurized hematoma, which prevents further blood loss until the wound can be surgically repaired. Methods: This study was approved by the SRCA Medical Services to be conducted at Al-Madenah. Data was collected on usage of the device from the prehospital through to patient disposition at the Emergency Department. The primary end points of the study are safety and efficacy of the iTClamp 50 in the prehospital setting. Results: Interim results after the enrolment of 4 patients are presented. In all cases, the indication was an actively bleeding wound to the scalp (2 cases) or limb (2 cases) caused by a motor vehicle accident or fall. In each case 1 iTClamp 50 was applied in the field and adequate hemostasis was achieved either immediately or within a few seconds with no subsequent re-bleeding. None of the patients required immediate blood transfusion before or at arrival in the emergency department. No adverse events were reported and pain was minimal upon application of the device. Conclusion: This study is ongoing until the enrolment of 20 patients. The interim results indicate that the iTClamp 50 is safe, effective, and efficient at controlling externally bleeding wounds when applied by SRCA emergency medical service providers. 